NON-LINEAR ACOUSTIC CHARACTERIZATION OF MICROBUBBLES AND NANOBUBBLES

By

Grace Fishbein

Bachelor of Science, Applied Math and Physics

Memorial University of Newfoundland, 2013

A thesis presented to Ryerson University

in partial fulfillment of the requirements

for the degree of

Master of Science

in the program of

Biomedical Physics

Toronto, Ontario, Canada, 2019

(C)Grace Fishbein, 2019 


\section{Declaration of Authorship}

I, Grace Fishbein, hereby declare that I am the sole author of this thesis. This is a true copy of the thesis, including any required final revisions, as accepted by my examiners.

I authorize Ryerson University to lend this thesis to other institutions or individuals for the purpose of scholarly research.

I further authorize Ryerson University to reproduce this thesis by photocopying or by other means, in total or in part, at the request of other institutions or individuals for the purpose of scholarly research.

I understand that my thesis may be made electronically available to the public.

Signed: 
"You must be shapeless, formless, like water. When you pour water in a cup, it becomes the cup. When you pour water in a bottle, it becomes the bottle. When you pour water in a teapot, it becomes the teapot. Water can drip and it can crash. Become like water my friend. "

Bruce Lee 


\title{
NON-LINEAR ACOUSTIC CHARACTERIZATION OF MICROBUBBLES AND NANOBUBBLES
}

\author{
Master of Science, Biomedical Physics \\ Grace Fishbein \\ Ryerson University, 2019
}

\begin{abstract}
Non-linear contrast-enhanced ultrasound can provide high contrast images by enhancing the non-linear signals from bubble oscillations. In this work, we developed a methodology to detect individual bubble scattering using focused transducers with dilute bubble solutions. Microbubbles and nanobubbles were made with five different lipid shell compositions. Their structure is altered through additional components added to the shell that affect their stability. Dilute samples of bubbles were sonicated at $25 \mathrm{MHz}$ with 30 cycles using a commercial high frequency ultrasound instrument with a pressure range of $75 \mathrm{kPa}$ to $3 \mathrm{MPa}$. Criteria were developed to ensure signals were only classified if they contained an isolated bubbles' response. The response of the bubbles of different shell compositions were compared using analysis tools developed. There were no observable differences in the non-linear behaviour between the different shells. However, when comparing microbubbles to nanobubbles differences involving signal count, stability and harmonic amplitudes were observed.
\end{abstract}




\section{Acknowledgements}

I begin by thanking my supervisor, Dr. Michael Kolios, for inviting me into his lab and taking a chance on me. I am very grateful for his support and guidance throughout this project. I also extend my thanks to my committee members, Dr. Karshafian, Dr. Tsai and Dr. Kirby for their scientific advice and encouraging feedback.

I am sincerely thankful for the contributions by Elizabeth Berndl, Amin J. Sojahrood, Niloufar Rostami-Shirazi and Hossein Haghi, all of whom are members of the Kolios lab. They assisted me tremendously by improving my understanding of the field and developing simulations and experimental techniques that were vital and necessary to complete this work.

To Morgan and Dana, thank you for your kindness, encouragement and endless support. I truly appreciate the generosity of your time and your gracious approach. It was an absolute pleasure to have ventured our master's degrees together.

To my Mom, thank you for always encouraging me to seek out the things that challenge me and for seeing me through them. I am eternally grateful for your life advice, your relentless love and support and for teaching me not to take myself too seriously. Your courage and determination inspire me every day. This accomplishment is as much yours as it is mine.

To Peter, I am forever grateful for your encouragement in pursuing this degree and making sure I saw it through no matter how challenging the circumstances. Thank you for pushing me to be a better version of myself everyday and for teaching me that actions speak louder than words. Your unconditional love and support give me the strength and confidence to achieve my goals. You are truly my anchor in life's ocean, my rock and my best friend. Thanks.

To all of my friends, family, and colleagues who have made this possible I extend a most sincere and heartfelt thank you. 


\section{Contents}

Declaration of Authorship ii

Abstract iv

Acknowledgements $\quad$ v

Contents vii

List of Tables $\quad$ xi

List of Figures $\quad$ xii

$\begin{array}{ll}\text { List of Abbreviations } & \text { xv }\end{array}$

List of Symbols $\quad$ xvi

1 Introduction 1

1.1 An Introduction to Ultrasound $\ldots \ldots \ldots \ldots$

1.2 Ultrasound Contrast Agents (UCAs) . . . . . . . . . . . . 2

1.2.1 An Introduction to UCAs . . . . . . . . . . . . . . . . 2

1.2.2 Understanding UCAs . . . . . . . . . . . . . . . . . 2

1.2.3 Diagnostic Imaging Applications of UCAs . . . . . . . . . . . . . 4

1.2.4 Therapeutic Applications of UCAs . . . . . . . . . . . . . . . . 4

1.3 Non-linear of UCA Response . . . . . . . . . . . . . . . . . . . . 6

1.3.1 Linear and Non-linear UCA Response . . . . . . . . . . . . . 6

1.3.2 Rayleigh-Plesset Equation for Bubble Dynamics . . . . . . . . . . . 7 
1.3.3 Resonant Frequency of a UCA _ . . . . . . . . . . . . . 7

1.3.4 UCA Shell Structure . . . . . . . . . . . . . . . . . . . . 8

1.4 Ultra-stable Microbubbles and Nanobubbles . . . . . . . . . . . . . . . . . 9

1.4.1 Introduction to Ultra-stable Bubbles . . . . . . . . . . . . . . . . 9

Shell Additive: Propylene Glycol . . . . . . . . . . . . . . . . . . . . . 10

Shell Additive: Glycerol . . . . . . . . . . . . . . . . . . . . . 11

Cross-linked Polymer Bubbles . . . . . . . . . . . . . . . . 11

1.4 .2 Nanobubbles . . . . . . . . . . . . . . . . . . . . . 12

1.4 .3 Surface Tension . . . . . . . . . . . . . . . . . . . 12

1.4 .4 Stability in vivo . . . . . . . . . . . . . . . . . 13

1.5 Thesis Overview $\ldots \ldots \ldots \ldots \ldots$

1.5.1 Engineering UCA Shell Design . . . . . . . . . . . . . . . 15

1.5 .2 Project Motivation . . . . . . . . . . . . . . . 15

1.5.3 Hypothesis and Specific Aims . . . . . . . . . . . . . . . 16

1.5.4 Expectated Behaviour of Different Shell Compositions . . . . . . . . 17

2 Materials and Methods: Experimental and Analytical 19

2.1 Microbubble and Nanobubble Production . . . . . . . . . . . . . . . . . . . 19

2.1.1 Initial Lipid Solution . . . . . . . . . . . . . . . . . . . . . . 19

2.1 .2 Bubble Activation . . . . . . . . . . . . . . . . . . . . . . 21

2.2 Size Isolation . . . . . . . . . . . . . . . . . . . . . . . . . 22

2.2 .1 Size Measurements . . . . . . . . . . . . . . . . . . . . . 22

2.2 .2 Microbubbles . . . . . . . . . . . . . . . . . . 22

2.2 .3 Nanobubbles . . . . . . . . . . . . . . . . . . . . 24

2.3 Single Bubble Scattering Experiments . . . . . . . . . . . . . . . . 25

2.3.1 Microbubble Experimental Protocol . . . . . . . . . . . . . . . . 26

2.3.2 Nanobubble Experimental Protocol . . . . . . . . . . . . . . 28

2.4 Algorithm for Signal Selection . . . . . . . . . . . . . . . . . . . . . . . . 29

2.4 .1 Criteria . . . . . . . . . . . . . . . . 30 
2.5 Signal Classification and Naming Procedure . . . . . . . . . . . . . . 34

2.5.1 The Classification of Accepted Signals . . . . . . . . . . . . . . . . 34

Linear Accepted Signals . . . . . . . . . . . . . . . . . . . . 34

Non-linear Accepted Signals . . . . . . . . . . . . . . . . . 35

2.5 .2 Rejected Signals . . . . . . . . . . . . . . . . . . . . . . . 42

2.5.3 Analysis of Classified Signals . . . . . . . . . . . . . . . 43

3 Results $\quad 44$

3.1 Size Measurements . . . . . . . . . . . . . . . . . . . . . . . . . 44

3.1 .1 Microbubbles . . . . . . . . . . . . . . . . . . . 44

3.1 .2 Nanobubbles . . . . . . . . . . . . . . . . . . . . 44

3.2 Signal Count Statistics . . . . . . . . . . . . . . . . . . 45

3.2.1 Initial Criteria Threshold . . . . . . . . . . . . . . . . . 45

3.2 .2 Signal Counts . . . . . . . . . . . . . . . . . . 50

3.2.3 Percent Total Count . . . . . . . . . . . . . . . . . . . . . . 50

3.3 Amplitude Analysis . . . . . . . . . . . . . . . . . . . . 53

3.3.1 Fundamental Amplitude Analysis . . . . . . . . . . . . . . . . 53

3.3.2 Subharmonic and Ultra Harmonic Amplitude Analysis . . . . . . . . 53

4 Discussion $\quad 66$

4.1 The Effect of Bubble Shell Composition on UCA Behaviour . . . . . . . . . . 66

4.1.1 Fundamental and Harmonic Amplitude Analysis . . . . . . . . . . 66

4.1.2 Size Distribution Dependence . . . . . . . . . . . . . . . . 67

4.2 A Comparison of Microbubbles and Nanobubbles . . . . . . . . . . . . . 67

4.2 .1 Initial Criteria Threshold $\ldots \ldots \ldots$. . . . . . . . . . . . . 67

4.2.2 A Statistical Comparison of Microbubbles and Nanobubbles . . . . . . 68

Non-linear Behaviour of Microbubbles and Nanobubbles . . . . . . . . 68

Bubble Bursting . . . . . . . . . . . . . . . . . . . 69

Period 3 Oscillations . . . . . . . . . . . . . . . . . . 71

4.2.3 Fundamental and Harmonic Amplitude Comparison . . . . . . . . . 73 
5 Conclusion and Future Work $\quad 76$

5.1 Conclusion . . . . . . . . . . . . . . . . . . . . . 76

5.2 Future Work . . . . . . . . . . . . . . . . . . . . . . 77

$\begin{array}{ll}\text { A Size Distribution Data } & 78\end{array}$

A.1 Microbubble Size Distribution . . . . . . . . . . . . . . . . . . 78

A.2 Nanobubble Size Distribution . . . . . . . . . . . . . . . . . . . . 78

B Additional Data $\quad 81$

B.1 Count of Burst Signals . . . . . . . . . . . . . . . . . 81

B.2 Average Difference between the Fundamental and Harmonic Maximum Amplitudes . . . . . . . . . . . . . . . . . . . 81

C MATLAB Codes $\quad 86$

C.1 Extract Code . . . . . . . . . . . . . . . . . . . . . . 86

C.2 Noise Threshold Code . . . . . . . . . . . . . . . . . . . . . 88

C.3 Automation Code . . . . . . . . . . . . . . . . . . . . . . . . . . 89

$\begin{array}{ll}\text { Bibliography } & 95\end{array}$ 


\section{List of Tables}

2.1 VEVO 770 Pressure (in MPa) from Percent Power Settings. . . . . . . . . . . . 26

3.1 Microbubble experimentally measured diameters and standard deviation. . . 44

3.2 Nanobubble experimentally measured diameters and standard deviation. . . 45 


\section{List of Figures}

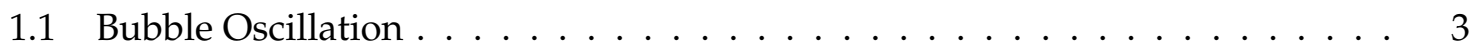

1.2 Shell Components . . . . . . . . . . . . . . . . . . . . . . . . . . . . 10

1.3 Surface Tension of Ultra-stable Bubbles f . . . . . . . . . . . . . . . . . . 13

1.4 in vivo Shell Comparison under Ultrasound Exposure . . . . . . . . . . . . . . 14

2.1 Microbubble Differential Centrifugation Steps . . . . . . . . . . . . . . . . 24

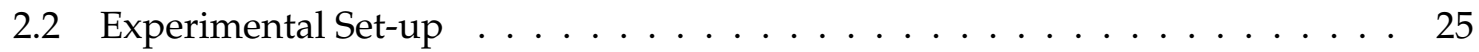

2.3 Experiment Screenshot . . . . . . . . . . . . . . . . . . . . . 27

2.4 Sample RF line: accepted bubble . . . . . . . . . . . . . . . . . . . . . . . . . . 29

2.5 Initial Threshold . . . . . . . . . . . . . . . . . . . . . . 30

2.6 Sample RF line: truncated bubble . . . . . . . . . . . . . . . . . . . . . . . . 31

2.7 Sample RF line: more than one bubble . . . . . . . . . . . . . . . 32

2.8 Noise Level . . . . . . . . . . . . . . . . . . . . . . . . 33

2.9 RF line Enveloped . . . . . . . . . . . . . . . . . . . . . . . . . . . . 33

2.10 Naming . . . . . . . . . . . . . . . . . . . 34

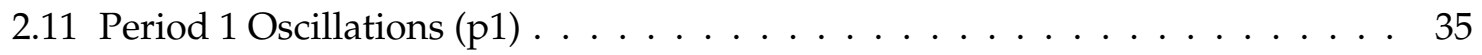

2.12 Period 2 Oscillations $(\mathrm{p} 2) \ldots \ldots \ldots \ldots \ldots \ldots \ldots$

2.13 Period 3 Oscillations $(\mathrm{p} 3) \ldots \ldots \ldots \ldots \ldots \ldots \ldots$

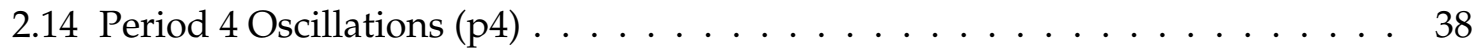

2.15 Period 5 Oscillations $(\mathrm{p} 5) \ldots \ldots \ldots \ldots \ldots \ldots$

2.16 Period 6 Oscillations $(\mathrm{p} 6) \ldots \ldots \ldots \ldots \ldots \ldots$

2.17 Period 7 Oscillations $(\mathrm{p} 7) \ldots \ldots \ldots \ldots \ldots \ldots \ldots$

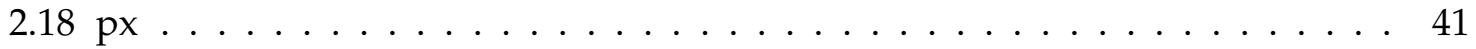


2.19 Burst Signals . . . . . . . . . . . . . . . . . . . . . . . . . 42

3.1 Microbubble Classified Signals by Experiment . . . . . . . . . . . . . . . 46

3.2 Nanobubble Classified Signals by Experiment . . . . . . . . . . . . . . . . 47

3.3 Microbubbles: Initial Criteria Threshold . . . . . . . . . . . . . . . . . . . . . . 48

3.4 Nanobubbles: Initial Criteria Threshold . . . . . . . . . . . . . . . . . . . 49

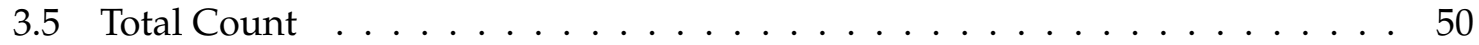

3.6 Non-linear Count . . . . . . . . . . . . . . . . . . . . . . . . . . 51

3.7 Microbubble Percent Non-linear . . . . . . . . . . . . . . . . . . . 52

3.8 Nanobubble Percent Non-linear . . . . . . . . . . . . . . . . . . . . . . . . . . . 52

3.9 Microbubble: p1 Fundamental Amplitude Analysis . . . . . . . . . . . . . . . 54

3.10 Nanobubble: p1 Fundamental Amplitude Analysis . . . . . . . . . . . . . . 55

3.11 Microbubble Non-linear Fundamental Amplitude Analysis . . . . . . . . . . 56

3.12 Nanobubble Non-linear Fundamental Amplitude Analysis . . . . . . . . . . . 57

3.13 Locating Harmonic Peaks . . . . . . . . . . . . . . . . . . 58

3.14 Subharmonic and Ultra Harmonic Peaks for p2, p3 and p4 . . . . . . . . . 59

3.15 Micro \#1: Fundamental, Subharmonic and Ultra Harmonic . . . . . . . . . . 59

3.16 Micro \#1: Fundamental, Subharmonic and Ultra Harmonic . . . . . . . . . . 60

3.17 Nano \#1: Fundamental, Subharmonic and Ultra Harmonic . . . . . . . . . . 60

3.18 Nano \#1: Fundamental, Subharmonic and Ultra Harmonic . . . . . . . . . . 61

3.19 Micro \#1: Harmonic Difference between Fundamental and Subharmonic . . . 62

3.20 Micro \#1: Harmonic Difference between Fundamental and Ultra Harmonic 63

3.21 Nano \#1: Harmonic Difference between Fundamental and Subharmonic . . . 64

3.22 Nano \#1: Harmonic Difference between Fundamental and Ultra Harmonic . . 65

4.1 First Criteria Threshold . . . . . . . . . . . . . . . . . . . . . . . . . 68

4.2 Total Non-linear Count . . . . . . . . . . . . . . . . . . . . . . . . . . . 69

4.3 Microbubble and Nanobubble: Percentage of Non-linear Signals . . . . . . . . 70

4.4 Total Burst . . . . . . . . . . . . . . . . . . . . . . . . . . 70

4.5 Microbubble and Nanobubble: Percent Burst Signals . . . . . . . . . . . . . . 71 
4.6 Bifurcation Diagram $\ldots \ldots \ldots \ldots \ldots \ldots \ldots \ldots \ldots$

4.7 Microbubbles and Nanobubbles: Total p3 signal count . . . . . . . . . . . 73

4.8 Microbubbles and Nanobubbles: p2 Fundamental Amplitude . . . . . . . . . 74

4.9 Microbubbles and Nanobubbles: p2 Ultra Harmonic Amplitude . . . . . . . . 75 


\title{
List of Abbreviations
}

\author{
UCA Ultrasound Contrast Agent \\ US Ultrasound \\ PG Propylene Glycol \\ G Glycerol \\ PGG Propylene Glycol and Glycerol \\ Xlink Cross Linked \\ p1 period 1 oscillation \\ p2 period 2 oscillation \\ p3 period 3 oscillation \\ p4 period 4 oscillation \\ p5 period 5 oscillation \\ p6 period 6 oscillation \\ p7 period 7 oscillation \\ px period Non-linear oscillation
}




\title{
List of Symbols
}

\author{
$\rho \quad$ bulk fluid density \\ $R \quad$ initial radius \\ $\dot{R} \quad$ bubble wall velocity \\ $\ddot{R} \quad$ bubble wall acceleration \\ $p_{g}(t)$ internal bubble gas pressure \\ $p_{0} \quad$ hydrostatic pressure \\ $p_{i}(t) \quad$ incident ultrasound pressure \\ $\eta \quad$ viscosity \\ $\chi \quad$ shell elasticity \\ $\sigma \quad$ surface tension \\ $f_{0} \quad$ resonant frequency \\ $\gamma \quad$ specific heat ratio \\ $\rho_{L} \quad$ density of the surrounding liquid
}


Dedicated To my Mom, my biggest inspiration 


\section{Chapter 1}

\section{Introduction}

\subsection{An Introduction to Ultrasound}

Ultrasound has long been a preferred diagnostic and therapeutic tool in the field of medicine because of its portability, safety, financial accessibility, and ability to image in real time. It has a wide range of utility in its diagnostic ability and treatment for the human body.

Ultrasound is the propagation of mechanical pressure waves of frequencies greater than $20 \mathrm{kHz}$ travelling through a medium. In the human body the medium is tissue. When these pressure waves come into contact with a medium that is has a different acoustic impedance, part of the wave will be reflected back, called backscatter, which is detected by the transmitting transducer [1]. The acoustic impedance of a medium is the product of the density and speed of sound within that material [2]. The distance of the object detected is calculated simply by using the speed of sound in the medium and the time that the wave takes to return to the transducer [3]. The frequency range of ultrasound used clinically is 1-20 MHz. The body has two materials that have significantly different acoustic properties from surrounding tissue these are: bone and the air in the lungs.

The contrast bone tissues provide can be seen in the ultrasound image of a fetus in utero, while bone also provides significant difficulties in using ultrasound to image the 
brain (due to the skull) or the spine. The lungs provide a challenge in using ultrasound imaging due to the large decrease in the speed of sound in air, impeding the ability to use ultrasound. Ironically, "imaging" air in the body using ultrasound ultimately opened the door to a whole new field of ultrasound research: Ultrasound Contrast Agents.

\subsection{Ultrasound Contrast Agents (UCAs)}

\subsubsection{An Introduction to UCAs}

A field of ultrasound imaging that requires improvement is the imaging of soft tissue contrast. Compared to other imaging modalities, ultrasound has poor soft tissue contrast. UCAs, also referred to as microbubbles and nanobubbles, are injectable, safe, and currently used clinically to increase contrast when imaging in the body. UCAs were discovered in the 1960s when Gramiak and Shah were investigating the aortic valve with ultrasound and they received unexpected contrast from the bubbles that formed from the dye they had injected [4][5][6]. This discovery led to the development of bubbles as UCAs.

Ultrasound may be replaced for another imaging modality with a higher contrast to tissue ratio when the contrast is not sufficient. Thus, there is substantial motivation to improve and enhance the use and stability of UCAs in ultrasound imaging [7].

\subsubsection{Understanding UCAs}

UCAs are gas filled shells typically smaller than $8 \mu \mathrm{m}$ in diameter to have the ability to travel through the smallest vessels in the body. Their shells are made of biocompatible materials so that they are safe for use within the body. The shells can be made of lipids, proteins (typically albumin), and/or polymers. For the purposes of this thesis, the focus will be on lipid shells which are used in most commercial UCAs [8]. The gas most commonly used in UCAs falls within the family of perfluorocarbons, due to their slower diffusion 
through the lipid shell and low solubility in water. The lipid shell is made of a layer of phospholipids which have a hydrophilic head and a hydrophobic tail. The initial stability of a bubble is due to this amphipathic structure as well as the contribution from Van der Waals forces that form naturally between the heads of phospholipids making up the outside shell of the microbubble. The phospholipid tails face the inside of a bubble because of their hydrophobicity. Lipid microbubbles are acoustically responsive due to this shell structure [9].

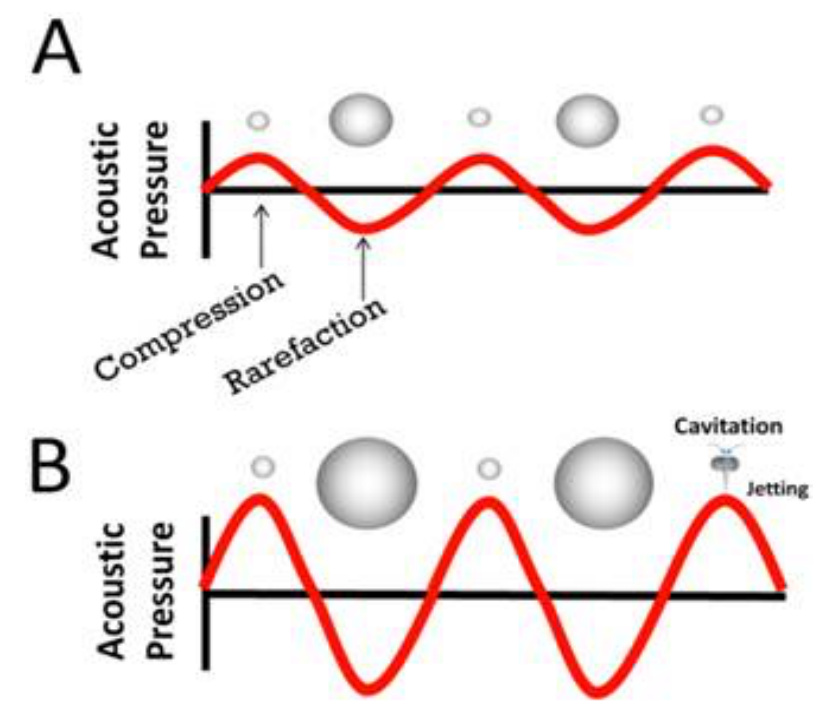

FIGURE 1.1: (A) A bubble undergoing stable oscillation when exposed to US.

(B) A bubble that is under too great a pressure and inertially cavitates (bursts) Adapted from [10].

Due to the significant difference in acoustic impedance of air versus tissue the encased air in a bubble offers a scattering source with increased echogenicity. A bubble increases ultrasound backscatter because of the compressibility of the gas encased in a malleable, deformable shell. As the mechanical pressure waves from the ultrasound probe travel through tissue and encounter a microbubble, a microbubble is then subjected to the compression and rarefaction components of the wave. As seen in Figure 1.2 the compression causes the microbubble to shrink in size as the gas inside the shell is compressed and the bubble radius decreases. In the rarefaction phase the microbubble expands and the radius increases in size. For each bubble size and shell design there is a limit to the pressure 
that a bubble can be exposed to before it will not be able to withstand any further deformation and the bubble will be destroyed as seen in Figure 1.2. This is referred to as inertial cavitation.

The compressibility of the gas inside a bubble and the elasticity of the shell of a bubble provides opportunity for non-linear response. Considerable contrast compared to tissue (which is a linear scatterer) can be achieved because of this unique behaviour. Tissue has a compressibility orders of magnitude smaller in comparison to bubbles and thus cannot provide the same type of non-linear signals that bubbles can [11] [12] [13]. A bubbles' non-linear response along with the ability to manipulate its size, shell structure and ability to attach to the shell is cellular targets offer many potential applications in diagnostic and therapeutic uses in the human body.

\subsubsection{Diagnostic Imaging Applications of UCAs}

UCAs are now commercially produced and commonly used in clinics. They are currently clinically approved to be used in echocardiograms, which help physicians image the blood flow through the heart, and imaging of the liver, which is used to identify tumours and other pathologies [14]. In order to be used as a contrast agent, a UCA needs to be stable between 5-30 min within the human body. This stability depends on the components and structure of the shell [4]. The approval of UCAs is specific to the country they are used in and the type of commercially available UCA being used.

\subsubsection{Therapeutic Applications of UCAs}

The application of UCAs is not limited to diagnostic imaging techniques in ultrasound. There is currently a significant number of researchers working on techniques 
for implementing therapeutic approaches using UCAs. One therapeutic application of microbubbles using ultrasound to increase macromolecule delivery through oscillating bubbles nearby cells. This is called sonoporation and has the potential to improve drug delivery and gene therapy by increasing tissue and cell permeability and subsequently localizing delivery [15]. Microbubbles undergoing oscillation can be used to induce apoptosis (programmed cell death) through increased stress on nearby cells [11]. More directly, a microbubble that is purposefully ruptured nearby cells, can cause significant damage, possibly irreversibly perforating the cell membrane causing cell death [15].

There are two main ways that microbubbles are thought to induce increased permeability: through jetting and microstreaming [9][16]. Jetting refers to fluid flow that occurs when a microbubble bursts. The burst creates a strong, fast, jet streamed current that travels at high speed in a relatively uncontrollable direction. The jetting impinging on a nearby cell can impart irreparable damage and allow for macromolecules to pass into the deeper tissue beyond the vasculature. Microstreaming is a method in which permeability can be increased in a less forceful way. Microstreaming refers to the motion induced on surrounding fluid as a direct result of microbubbles undergoing steady oscillation [10]. This can encourage the uptake of a drug or DNA through endocytosis by nearby cells or by transiently opening pores on the cell surface [10].

There is another effect the oscillations of microbubbles have on the extracellular matrix of blood vessels. The vasculature responds to pressure changes within the walls of a vessel. Thus, if an oscillating microbubble changes the local pressure, this can generate gaps between cells, at cell junctions, creating an opportunity for particles and macromolecules to pass through. This is termed extravasation [10].

Microbubbles can also be used as drug delivery vehicles in the body. This requires loading the shells of microbubbles with a desired drug to be delivered. Targeted microbubbles are injected into the body and at high pressures, rupture [11]. The microbubble shells break into fragments releasing the macromolecule locally and reducing the systemic effects 
[9]. The loading of the drug or gene into the microbubble can be done multiple ways, the specifics will vary depending on the type of drug or gene [9]. This brings up two important points regarding the desired function of this drug delivery system. First, the loading of a macromolecule into the shell of a bubble will most likely alter its acoustic response to ultrasound. Secondly, inertial cavitation of the bubble should entirely release the desired macromolecule in the target area to maximize localized drug delivery.

Furthermore, targeted ligands can be attached to a UCA to encourage it to connect to biological structures within the body thus reducing its movement and restricting it to a specific location. It is important to understand how additional shell elements, such as ligands, change a UCA's stability, size, and ultrasound response [17]. Some researchers investigate how to attach a microbubble to endothelial cells in the vasculature so that their contents could be released over a longer duration of time [9].

Commercially available contrast agents that are currently on the market were designed and optimized for diagnostic applications. The different shell characteristics needed for diagnostic and therapeutic applications of microbubbles highlight the need to engineer UCAs for the required task [18].

\subsection{Non-linear of UCA Response}

\subsubsection{Linear and Non-linear UCA Response}

A brief explanation for linear versus non-linear behaviour as it pertains to bubble oscillations is provided. A linear bubble oscillation will have a consistent minimum and maximum shell radius throughout the ultrasound excitation. For non-linear oscillations the minimum and maximum shell radius will vary with time and the amplitude will deviate from the US excitation pattern. Generally, UCA's will demonstrate a linear response to low acoustic pressures and a non-linear response to higher acoustic pressures [6]. It is necessary 
to know the specific shell size and properties to quantify their response to low and high acoustic pressures.

\subsubsection{Rayleigh-Plesset Equation for Bubble Dynamics}

Equation 1.1 shows the fundamental theory for bubble dynamics which was demonstrated by Rayleigh and Plesset [19] [6] [17]. It offers a theoretical non-linear explanation of the dynamics of a bubble surrounded by liquid in response to a pressure field [19]. It assumes uniform gas pressure, neglects liquid compressibility and obeys the polytropic law [17].

$$
\rho\left(\ddot{R} R+\frac{3}{2} \dot{R}^{2}\right)=p_{g}(t)-p_{0}-p_{i}(t)-\frac{4 \eta \dot{R}}{R}-\frac{2 \sigma}{R}
$$

$\rho$ is the density of the bulk fluid. $R_{0}$ is the initial bubble radius. $\dot{R}$ and $\ddot{R}$ are the first and second order derivatives of the radius respectively. $p_{g}(t)$ is the uniform gas pressure, $p_{0}$ is the hydrostatic pressure and $p_{i}(t)$ is the ultrasound pressure. $\eta$ is the viscosity of the bulk fluid and $\sigma$ is the surface tension [17]. Solutions to this equation can provide insight into how a bubble responds to external excitation [17].

\subsubsection{Resonant Frequency of a UCA}

Resonance occurs when an external force is applied to a system at a rate that is equal to the natural frequency of the system. A bubble will demonstrate prolonged increased amplitude of oscillation when sonicated at its' resonance frequency. Equation 1.2 [8] shows that the resonant frequency of a bubble is determined by its' initial radius and shell elasticity. 


$$
f_{0}=\frac{1}{2 \pi R} \sqrt{\frac{1}{\rho_{L}}\left[3 \gamma P_{0}+\frac{2(3 \gamma-1) \sigma}{R}+\frac{4 \chi}{R}\right]}
$$

As the size of a microbubble increases, its resonant frequency decreases. Parameters such as shell structure come into consideration as the elasticity, specific heat capacity and surface tension to determine a microbubble's behaviour. The initial bubble radius is denoted by $R$ and the resonant frequency by $f_{0}$. The other symbols in this equation include the shell elasticity, $\chi$; the surface tension at the gas-liquid interface, $\sigma$; the ambient fluid pressure, $P_{0}$; the density of the surrounding liquid, $\rho_{L}$; and the specific heat ratio, $\gamma$ [12]. In order to compare individual bubble oscillations it is important that the size range of the bubbles in the experimental sample be as small as possible (i.e. have size uniformity), since the behaviour and response of a UCA is largely dependent on size [4].

\subsubsection{UCA Shell Structure}

A bubble without a shell is labeled as a free bubble. A free bubble model is the first step to understanding and simulating bubble behaviour. However, a bubble lacking a shell is not practical as the gas would quickly diffuse. The shell of a bubble is vital to its stability before, during and after ultrasound exposure. The structure of bubbles' shell also allows for buckling, which is critical for non-linear behaviour, prevents coalescence and changes the surface tension.

Adding a shell to a free bubble increases stability. The shell prevents the gas from escaping out of the bubble into the surrounding medium. Also, the shell prevents the bubbles from coalescing and possibly forming bigger bubbles with increased gas and excess shell materials [4].

Buckling is a reversible phenomena that occurs within a bubble shell while under the compression phase of oscillation or under high pressure [20]. A bubble's shell will fold itself, similar to wrinkles, to allow the shell to shrink and remain intact while the gas is 
being compressed. Buckling is important as it alters properties of the shell such as elasticity and surface tension. The surface tension drops close to zero when the shell has buckled [20]. Furthermore, it should be noted that surface tension is expected to decrease as the bubble size decreases [21].

Part of the challenge is designing the optimal shell for the desired application. A bubbles' shell needs to be thick enough to provide adequate stability and prevent diffusion of gas but not so thick that it drastically changes the behaviour of the bubble [22]. Typically a bubble with a thinner shell will provide a stronger backscatter signal compared to a thicker shell [23]. All are important considerations for shell engineering.

\subsection{Ultra-stable Microbubbles and Nanobubbles}

\subsubsection{Introduction to Ultra-stable Bubbles}

Dr. Exner's lab, at Case Western Reserve University in Cleveland, Ohio, have developed ultra-stable lipid nanobubbles that have been shown to maintain longer periods of contrast under ultrasound exposure than that of a commercially available UCA [24]. The bubble protocol that is followed in this thesis (described in detail in the methods section) is used to synthesize the bubbles and shared through a joint collaboration with Dr. Exner's lab.

The ultra-stable protocol can also be used to create microbubbles and nanobubbles of the same shell composition. The shell contains a base lipid structure with two additional biocompatible elements that increase overall stability and applicability of these bubbles: propylene glycol (PG) and glycerol (G) [24]. The Exner lab made 4 different shelled bubbles. The first, contained only lipids and no shell additives, the second contained the basic lipid structure as well as the addition of propylene glycol, the third contained the basic lipid structure and the addition of glycerol and the final shell type is a composition of the basic 

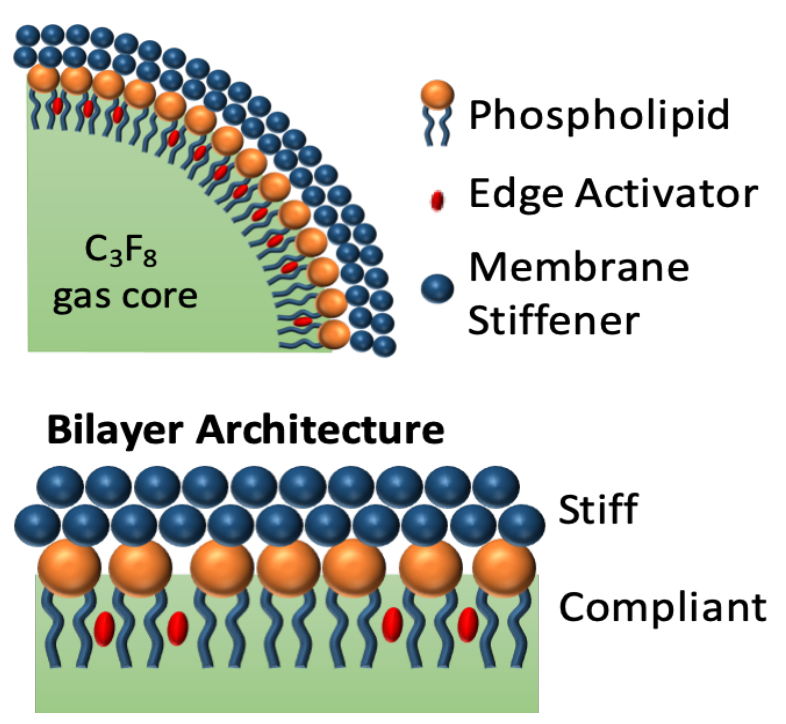

FIGURE 1.2: A depiction of the addition of an Edge-Activator (propylene glycol) and a membrane stiffener (glycerol) within a lipid shell structure. Adapted from [24].

lipid structure, propylene glycol and glycerol (PGG). A simple illustration of the shell's components can be seen in Figure 1.2. It was determined through these experiments that the most stable shell under ultrasound exposure was the shell composed of lipids, propylene glycol and glycerol. This combination of enhanced elasticity with the propylene glycol and increased stiffness with the glycerol offered the slowest signal decay and the longest in vivo half-life [24].

\section{Shell Additive: Propylene Glycol}

Propylene glycol is an edge-activator, which acts to enhance the flexibility of a bubble's shell, offering increased stability [24]. As demonstrated in work with ultra-deformable liposomes, the addition of an edge-activator to a basic lipid bound particle (buoyant or nonbuoyant) increases it's ability to contort itself to move through a narrow passage without affecting the structural integrity of the particle [25]. In a comparison between lipid bubbles with and without PG, Dr. Exner's lab's work suggests that in some positions in the shell the distances between phospholipids increases by 2.5 times their distance when PG is not 
present in the shell [24]. The addition of PG to a bubble shell gives it the ability to undergo significant transformations in a very short period of time and not destruct. PG adds increased stability to the bubble during non-linear oscillations [24].

\section{Shell Additive: Glycerol}

Glycerol is a viscous substance that is used in stabilizing UCAs by increasing stiffness in the shell. Abou-Saleh et al. discovered that the addition of glycerol to lipid-shelled microbubbles contributes to a narrower size distribution and prolonged stability. This suggests that the increase in glycerol concentrations in the bubble solution volume limits the permeability of the gas leaking outside the bubbles' shell [26].

\section{Cross-linked Polymer Bubbles}

In 2016 Dr. Exner's lab developed another type of bubble with a shell composed of lipids and a cross linked polymer network. These bubbles were made using an older protocol and formulation compared to the recently developed ultra-stable nanobubbles. In this thesis, we termed these Xlink bubbles and they were made from an updated protocol available in section 2.1.

Experiments with the Xlink bubbles showed increased echogenicity in vivo and increased extravasation compared to microbubbles [27]. The enhanced echogenicity could be accounted for by the increased elasticity in the shell of Xlink bubbles. The Xlink bubble may be more efficient at preventing gas diffusion as they were shown to have a slower rate of decay than nanobubbles that did not have crosslinked polymers in the shell. 


\subsubsection{Nanobubbles}

Typically UCAs are considered nanobubbles if they have a diameter smaller than $1 \mu \mathrm{m}$. Although research in nanobubbles is relatively new, they have been shown to have advantages over microbubbles. Dr. Exner's lab made nanobubbles using their protocol for ultra-stable bubbles and they were compared to commercially available Definity microbubbles. In vitro and in vivo experiments were done in vivo at Case Western Reserve University. In these experiments nanobubbles were found to have higher echogenicity in vitro and slower decay rates in vivo compared to Definity [28]. An explanation for this is the nanobubbles' capacity to extravasate beyond the vasculature is much greater than that of microbubble due to the nanobubbles' smaller size [24] [28]. Therefore, they would remain in the target tissue longer.

\subsubsection{Surface Tension}

The surface tension of the shell of a bubbles will affect the bubbles' stability and non-linear behaviour. Typically a lower surface tension indicates increased stability. Figure 1.3 shows a difference in surface tension for each of the different types of interfaces using three different methods. The pendant drop method is an experiment that examines the largest possible droplet of the liquid that makes up the bubbles shell. The droplet is imaged as it grows and the point of last contact is measured to determine surface tension just before the drop is released due to being overcome by the force of gravity. The other two methods used in this experiment to measure surface tension are called the rising drop method. This requires the use of a gas rising through a liquid with the measurement taken at the point of last contact before the bubble is released. This method was done two ways, one had a bubble made with air as the gas inside and the other used $C_{3} F_{8}$ as the gas inside [24]. Dr. Exner's lab found that although there are differences in the surface tension measurements the lowest surface tension didn't necessarily correlate to that type of shell having the highest 
stability.

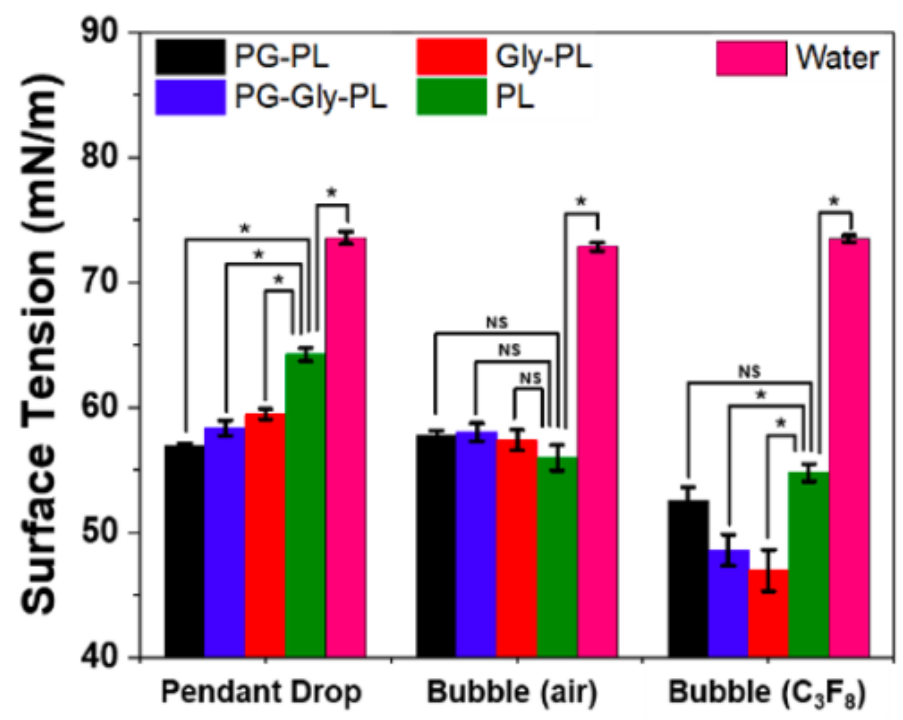

FIGURE 1.3: Measurements of surface tension were made on each type of shell (except Xlink) using different measurement methods. The pendant drop method was used and the rising drop method was used with both air and $\mathrm{C}_{3} F_{8}$. Adapted from [24].

\subsubsection{Stability in vivo}

Dr. Exner's lab performed in vivo experiments with the ultra-stable nanobubbles to investigate the difference in stability for the different shell compositions. The results of these experiments is shown in Figure 1.4 which was adapted from the published article [24]. It was found that PGG nanobubbles (referred to in Figure 1.4 as PG-Gly-PL) exhibited prolonged increased contrast in comparison to the other bubble shell compositions including a commerically available UCA, Lumason.

\subsection{Thesis Overview}

Changing the shell of a UCA impacts three main microbubble parameters; the range of sizes, the non-linear response and the stability of the UCA when it is exposed 


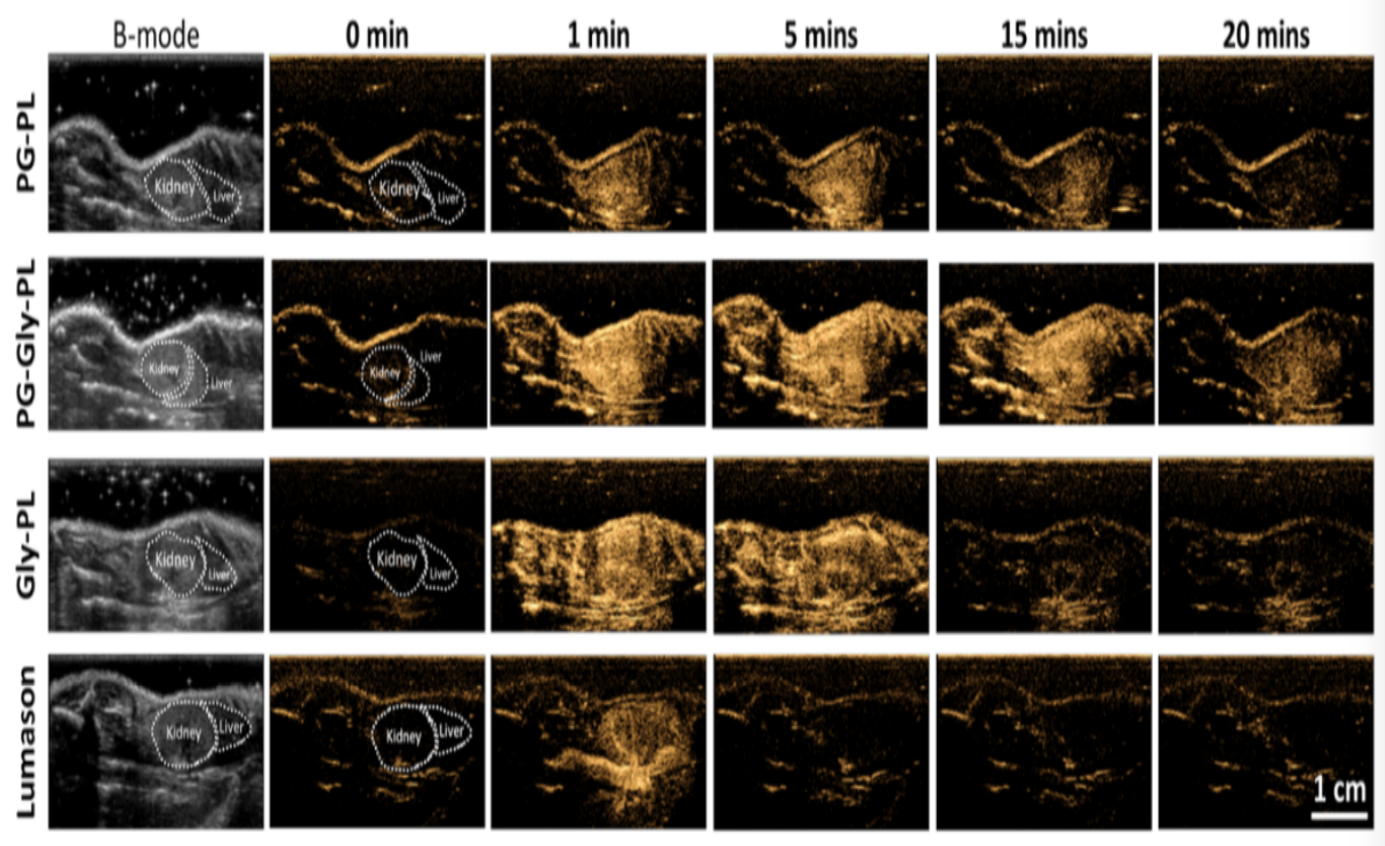

FIGURE 1.4: Cross-sectional images of a mouse kidney and liver, with nanobubbles administered using tail-vein injection. PG-PL is propylene glycol and lipid shell. PG-Gly-PL is propylene glycol, glycerol and lipid shell. Gly-PL is glycerol and lipid shell. Lumason is a commercially available lipidshelled microbubble. The B-mode images are before injection and the contrast enhanced ultrasound images moving to the right are after injection (time point at top). Prolonged increased contrast from the PG-Gly-PL bubbles versus the other shells, demonstrate the stability of PG-Gly-PL bubbles under prolonged ultrasound exposure. Adapted from [24]. 
to ultrasound [26]. Understanding how the UCA shell composition affects its behaviour and simulating these accurately with UCA theory helps us understand how to design the optimum shell for the desired UCA behaviour for a particular application [29].

\subsubsection{Engineering UCA Shell Design}

Since a bubbles' behaviour under ultrasound will depend on its size (see Equation 1.2) it is important to consider the range of size within a microbubble population. The more narrow a size distribution the easier it will be to determine the best ultrasound parameters (frequency and pressure) to target a sample of bubbles. Another consideration is the permeability of the shell's effect on gas diffusion during oscillation [26]. Furthermore, the concentration and bubble stability are important in designing the ideal UCA [29].

In a recent article describing engineering UCAs, the author proposes applying approaching bubble design from the perspective of reverse engineering [4]. Reverse engineering requires first obtaining answers to the questions about the practical function of a product in order to reach the desired completion of said product that completely satisfies all previous challenges. The argument is that it assists in reaching a formidable conclusion, in this case, UCAs that behave optimally for the task required.

Current clinically approved applications require that commercially available UCAs were made with the purpose of being used as contrast agents. Since the therapeutic uses of UCAs are promising, researchers are repurposing commercially available UCAs for therapy. The value of making in-lab bubbles lies in the knowledge and control of what materials go into them. Additionally, it is advantageous to design them for specific needs [4].

\subsubsection{Project Motivation}

The non-linear response of microbubbles is critical to the contrast that bubbles generate in contrast-enhanced ultrasound. Understanding how shell parameters effect the 
non-linear response is important. If experimental shell manipulation can be controlled and optimized, bubbles could be rationally designed for specific applications.

This project is driven by the motivation to develop a more thorough understanding of how the change in shell properties effect UCA non-linear oscillations and stability. This work attempts to take a step closer to understanding the effect of changing shell parameters on their stability and non-linear response, in addition to how these change with increasing pressure. The methods of the analysis chosen focuses on non-linear behaviour of UCA's.

Throughout this work, two different sizes of bubbles were used, referred to as Microbubbles and Nanobubbles. Both sizes of bubbles were made using five different shell compositions. All shell compositions have the same base components in the lipid material but each has slightly altered additional components. For each bubble size and specific shell design single bubble backscatter experiments were performed. These experiments were adapted based off a technique introduced by Omar Falou who examined the ultrasound backscatter from single cells and characterized them based on theoretical predictions from a fluid sphere model [30]. The aim of the single bubble backscatter experiments in this work is to observe any changes in non-linear behaviour between different shell compositions for microbubbles and nanobubbles as a function of increasing pressure.

\subsubsection{Hypothesis and Specific Aims}

\section{Hypothesis}

Single bubble backscatter experiments can be used to compare non-linear behaviour for microbubbles and nanobubbles of different shell compositions.

\section{Specific Aims}

To compare the non-linear behaviour, the specific aims of this thesis are: 
1. Make microbubbles and nanobubbles of five different shell types: Lipids, PG, G, PGG and Xlink.

2. Develop a methodology to carry out single bubble backscatter experiments for microbubbles and nanobubbles to determine their pressure dependence and non-linear response.

3. Design an algorithm that performs analysis on isolated bubble oscillation backscatter data.

\subsubsection{Expectated Behaviour of Different Shell Compositions}

A brief summary of the proposed expectations of the different shell behaviour is included. These expectations are based off of the previous work done by Dr. Exner's lab. The PGG bubbles, have the longest sustainable oscillation stability under ultrasound, in vivo [24]. It was anticipated that the PGG bubbles would hold the most consistent performance throughout the methods of analysis used in this work. PGG bubbles would show the largest degree of non-linear behaviour with increasing pressure with minimal destruction (for both microbubbles and nanobubbles). The PG bubbles were considered to have the highest range of controlled flexibility, meaning they can undergo major shell distortion and resume their previous shape. It is predicted that they will exhibit non-linear behaviour at a lower pressure threshold (relative to other bubble shells) due to this increased flexibility. This non-linear behaviour will continue up to a certain threshold. Past this threshold the bubbles will not have the stability to maintain non-linear oscillations without bursting. Lipid bubbles are expected to also have strong non-linear response beyond a certain threshold after which many will undergo inertial cavitation. The main difference between the expected behaviour of the PG and the Lipid bubbles is that the PG bubbles would be expected to have a higher pressure threshold than the Lipid bubbles due to the additional of propylene glycol which contributes to the shell reformation ability. The G bubbles are considered the stiffest bubbles in this work and thus were predicted to have the highest 
stability. The viscosity of the shell will be greatest and will cause increased damping [31]. Lastly, the Xlink bubbles are expected to demonstrate increased non-linear behaviour due do their increased elasticity. They should also be expected to have a decreased rate of destruction as shown by their prolonged stability under ultrasound exposure [27]. 


\section{Chapter 2}

\section{Materials and Methods: Experimental and Analytical}

\subsection{Microbubble and Nanobubble Production}

\subsubsection{Initial Lipid Solution}

All of the microbubbles and nanobubbles used in this work were made, in lab, following the published protocol for the production of ultra-stable bubbles [24]. Five different shell types were made with their naming referring to the additional elements added to the shell: lipid bubbles (denoted as Lipid), lipid bubbles with glycerol (denoted as G), lipid bubbles with propylene glycol (denoted as PG), lipid bubbles with propylene glycol and glycerol (denoted as PGG) and lipid bubbles with propylene glycol, glycerol and three additional chemicals that under UV light assist in UV polymerization (denoted as Xlink).

The protocols to make these microbubbles and nanobubbles differ only slightly across the different types of shells used in this work. The first step is to retrieve the lipids from the freezer. Next, a water bath is prepared at a temperature of $80^{\circ} \mathrm{C}$ and the sonicator (1510 Branson, Branson Ultrasonics Corp., CT, USA) is prepared to be used at room temperature. Once the lipids have reached room temperature they are each measured and put in a 
$20 \mathrm{~mL}$ glass vial (Fisherbrand ${ }^{\mathrm{TM}}$, PA, USA). For a total solution volume of $10 \mathrm{~mL}$ (and thus 10 individual vials containing $1 \mathrm{~mL}$ of solution) the following mixtures are used: $60.1 \mathrm{mg}$ of DBPC (1,2-dibehenoyl-sn-glycero-3-phosphocholine) (Avanti ${ }^{\circledR}$, Sigma-Aldrich, MO, USA), $10 \mathrm{mg}$ of DPPA (1,2-dipalmitoyl-sn-glycero-3-phosphate, sodium salt) (Avanti ${ }^{\circledR}$, SigmaAldrich, MO, USA), 20 mg of DPPE (1,2-Dipalmitoyl-sn-glycero-3-phosphoethanolamine) (CordenPharma, CO, USA), and 10 mg of DSPE-mPEG(2000) (1,2-distearoyl-n-glycero-3phosphoethanolamine-N-[methoxy(polyethylene glycol)-2000](ammonium salt)) (Avanti ${ }^{\circledR}$, Sigma-Aldrich, MO, USA).

Each lipid was measured separately on weighing paper (Fisherbrand ${ }^{\circledR}$, PA, USA) using an enclosed electronic scale (Scientech, CO, USA). The next step in the protocol depends on which type of bubble shell is being made. For the lipid bubbles (which have lipids only) $10 \mathrm{~mL}$ of PBS (Wisent Bioproducts, QC, Canada) is added to the vial containing the powdered lipid mixture. For the G bubbles $2 \mathrm{~mL}$ of glycerol (Sigma-Aldrich, MO, USA) is added to the vial. The PG bubbles require $2 \mathrm{~mL}$ of propylene glycol (Fisher Scientific, NJ, USA) added to the vial. For the PGG and Xlink bubbles, $1 \mathrm{~mL}$ of propylene glycol was added. All the vials are placed in the water bath for a minimum of 15 minutes. If the solution still contains small chunks of lipids after 15 minutes the vial is placed back into the sonicator for 10-20 seconds, every couple of minutes, returning it to the water bath in between.

If no lipid chunks are observed and the solution has a milky appearance then a homogeneous solution is achieved, a second $20 \mathrm{~mL}$ vial is used to prepare and warm (using the water bath) the remaining volume to be added to the lipids. For the G and PG bubbles this is $8 \mathrm{~mL}$ of PBS. The PGG and Xlink bubbles require a combination of $1 \mathrm{~mL}$ of glycerol and $8 \mathrm{~mL}$ of PBS. These are measured and put together in the same $20 \mathrm{~mL}$ vial and then mixed using a vortex mixer (VWR TM, Avantar, NJ, USA) with speeds sufficient to remove any visibility of the presence of glycerol within the PBS but not so fast as to introduce bubbles into the vial. This second vial is placed in the water bath for several minutes to be 
heated to the same temperature as the original vial. Then for all bubble shell types the contents of the second vial are slowly pipetted into the lipid vial in $1 \mathrm{~mL}$ increments, swirling gently after each amount is added. After this transfer is complete the vial containing everything is placed in the sonicator for ten minutes to ensure a homogeneous solution by breaking up any excess lipid particles. The sonication time of the vial should be increased if any chunks of lipids are present.

The final step before the bubbles are activated is to take the lipid solution and aliquot the $10 \mathrm{~mL}$ into $1 \mathrm{~mL}$ amounts, each into a $3 \mathrm{~mL}$ vial (Wheaton, NJ, USA) which is then capped with a rubber cap (Wheaton, NJ, USA) and topped with aluminum (Wheaton, NJ, USA) which is sealed with a capping device (E-Z CRIMPER TM, Wheaton, NJ, USA). These vials were then stored at room temperature for same day activation for all experiments in this work.

\subsubsection{Bubble Activation}

The next step is to activate the bubbles. One of the $3 \mathrm{~mL}$ vials is needed for bubble activation. First a gas exchanged must be performed. A $23 \mathrm{G}$ needle (Becton Dickinson (BD), NJ, USA) and a $20 \mathrm{~mL}$ syringe (Becton Dickinson (BD), NJ, USA) is used to pull gas out of the vial 5 times, creating as close as possible to a vacuum inside the vial. Next a $10 \mathrm{~mL}$ syringe (Becton Dickinson (BD), NJ, USA) is filled with octafluoropropane $\left(C_{3} F_{8}\right)$ (Synquest Labratories, FL, USA), with a $23 \mathrm{G}$ needle attached to this syringe. The rubber cap of the vial is then punctured allowing pressure to equalize. The rubber seal is punctured with a second needle so that it is vented and the syringe filled with octafluoropropane is depressed. Once the syringe is fully depressed, both needles are removed from the vial at the same time. With the gas exchange complete the vial is placed on its side in a mechanical shaker (Bristol Myers Squibb (BMS), NY, USA) for 45 seconds. The bubbles are now activated.

The Xlink bubbles are made by modifying already activated PGG bubbles using the procedure described above. Before the vials are capped, three chemicals are added to 
cause UV polymerization. These include: 5mg of Irgacure (2-Hydroxy-4'-(-2-hydroxyethoxy)2-methylpropiophenone) (Sigma-Aldrich, MO, USA), 34 uL of NNDEA (N,N-Diethylacrylamide) (Sigma-Aldrich, MO, USA), and 5mg of BAC (N,N'-Bis(acrykoyl)cystamine) (Beantown Chemical (BTC), NH, USA). The vial is then capped and sealed. This vial is then activated as described in section 2.1.2. The sealed cap is very quickly removed off the activated $3 \mathrm{~mL}$ bubble vial to place a small stir bar inside. This vial is then recapped as quick as possible to reduce octafluoropropane gas escaping the vial. The vial is once again flooded with octafluoropropane gas. The vial is placed under UV light (Spectroline, NY, USA) at $257 \mathrm{~nm}$ for 30 mins with mixing throughout at approximately $53 \mathrm{rpm}$, flipping the vial at 15 minutes. The bubbles are now ready to be used.

\subsection{Size Isolation}

\subsubsection{Size Measurements}

The size measurements for the Microbubbles were performed using the Coulter Counter (Beckman Coulter, IN, USA) and the size measurements for the nanobubbles were done using the Archimedes Particle Metrology System (Malvern Panalytical, MA, USA).

\subsubsection{Microbubbles}

The microbubbles were isolated to a size of approximately $1-2 \mu \mathrm{m}$ in diameter through multiple centrifugation steps [32]. Three activated vials were used to isolate the population of microbubbles to increase the yield, since there is a significant reduction of the concentration of microbubbles through this size isolation. A beaker of $100 \mathrm{~mL}$ of PBS is prepared. All bubbles are drawn out from each vial with a $23 \mathrm{G}$ needle and a $3 \mathrm{~mL}$ syringe with a second $23 \mathrm{G}$ needle puncturing the cap to vent it. The bubbles are diluted in the beaker. The solution is swirled slowly to mix the bubbles in the PBS. Eight $30 \mathrm{~mL}$ syringes 
(Becton Dickinson (BD)) (without a needle attached) are used to draw out $4 \mathrm{~mL}$ of the diluted bubble solution. Para-film is used to cover the tip of the syringe. The eight syringes are placed in the centrifuge (plungers up) for 2 minutes at $50 \mathrm{RCF}$. When the syringes are finished in the centrifuge the para-film is removed from each one. Within the syringe there will be a visible difference between the larger bubbles that have risen to the top and the rest of the bubbles. These larger bubbles are termed the cake and will appear more white and opaque than the rest of the solution. Initially most of the contents of the syringes are emptied into a "waste beaker", these are the smaller bubbles being removed. The plunger on the syringe is depressed until just before reaching the "cake" the larger white bubbles. The remaining bubbles in the syringe are depressed into a second beaker containing $50 \mathrm{~mL}$ of PBS. After all of syringes are emptied then the process is repeated. The syringes are again each filled with $4 \mathrm{~mL}$ of the new solution with the larger bubbles and then centrifuged for 2 minutes at a speed of $160 \mathrm{RCF}$. The smaller excess bubbles are once again removed from the syringes and the cake is saved and diluted into a 4th beaker containing $50 \mathrm{~mL}$. The syringes are used to draw up the larger bubble solution for a third time, this time centrifuging for 2 minutes at $180 \mathrm{RCF}$. This process is repeated one last time to eliminate the smaller bubbles in the waste beaker and then save the "cake" into a beaker containing $25 \mathrm{~mL}$. This solution is once again drawn up and centrifuged for 1 minute at a speed of 250 RCF. The small bubbles are eliminated one last time and the larger bubbles are diluted into $20 \mathrm{~mL}$ of PBS in a beaker and then transferred to a $20 \mathrm{~mL}$ vial. This protocol was developed in the Kolios lab by Niloufar Rostam-Shirazi, an undergraduate student. It was adapted from the work in Mark Borden's lab where they developed the technique of microbubble size isolation by differential centrifugation [32] and also from Dr. Borelli's lab where uniformly sized serum albumin and dextrose microbubbles were produced [33]. Figure 2.1 shows an example of two syringes throughout the differential centrifugation process. One is shown after the first centrifugation step at $50 \mathrm{RCF}$ and the second is shown at the third centrifugation step at 180 RCF. 

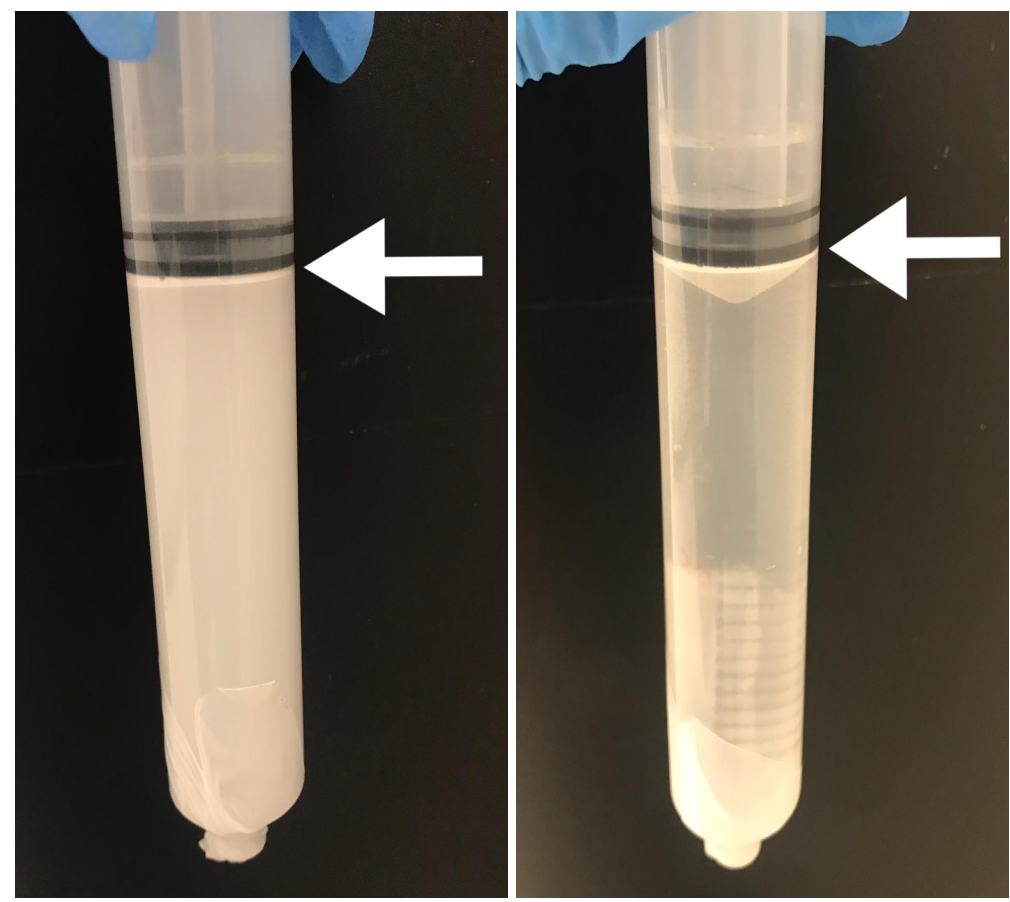

FIGURE 2.1: Post centrifugation visual of the separation between the larger and smaller bubbles shown by the white arrow. The image on the left was taken after the first centrifugation step at $50 \mathrm{RCF}$ and the image on the right was taken after the third centrifugation step at 180 RCF.

The bubble sizes and concentrations are then measured using the Beckman Coulter. $1 \mathrm{~mL}$ of the isolated bubble solution is pipetted and diluted in $20 \mathrm{~mL}$ of isotone and measured with the Coulter counter. The $30 \mu \mathrm{m}$ aperture is used and a water blank is subtracted from the measured sample.

\subsubsection{Nanobubbles}

The nanobubbles for this work were isolated to approximately $250 \mathrm{~nm}$ in diameter through differential centrifugation [24]. The vial of $1 \mathrm{~mL}$ bubble solution is placed inverted in the centrifuge (MXIE TD5A-WS) at 50 RCF for 5 mins. When the vial is removed from the centrifuge it is kept inverted. A $1 \mathrm{~mL}$ syringe (Becton Dickinson (BD)) is used to penetrate the rubber cap on the vial to extract the bubbles. The cap of a needle that has been cut off and then placed back on the needle to permit accurate penetration depth and therefore select bubbles of a particular size. 


\subsection{Single Bubble Scattering Experiments}

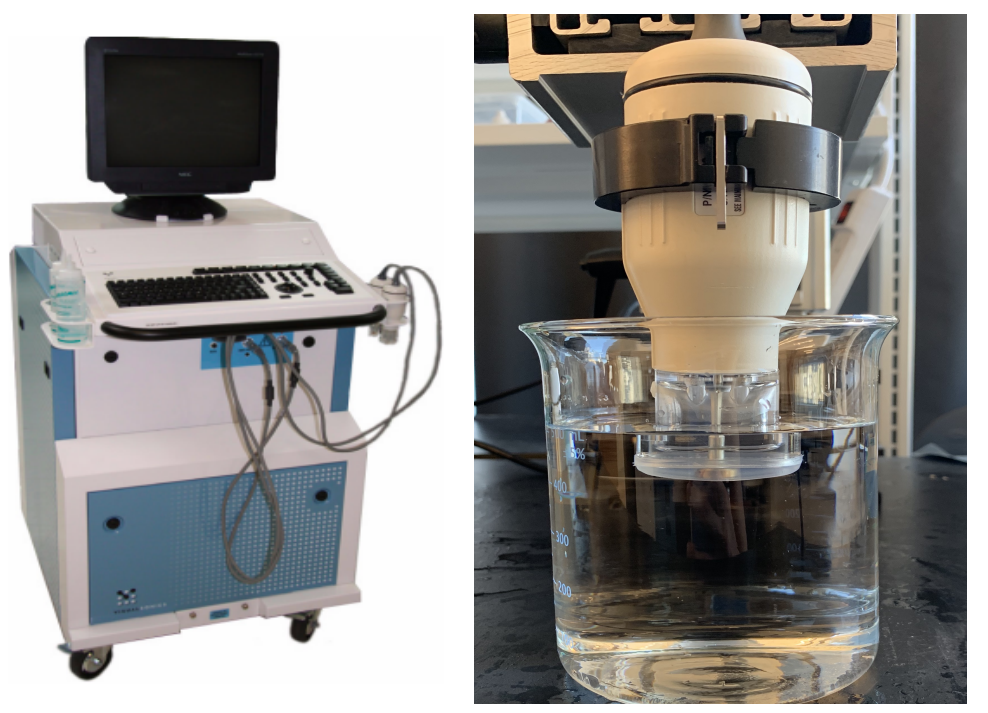

FIGURE 2.2: VEVO 770 and the RMV-710B focused transducer with a mechanical scanhead held with an adjustable clamp in a beaker containing 500 $\mathrm{mL}$ of DI water [34]

The bubble backscatter experiments were completed using the Vevo 770 using methods developed in Falou's work mentioned in section 1.5.2 [30]. The transducer used is the RMV-710B (f-number 2.1; aperture $7.14 \mathrm{~mm}$; focal length $15 \mathrm{~mm}$ ), this is a focused transducer with a center frequency of $25 \mathrm{MHz}$ and a 100\% bandwidth that oscillates back and forth on a mechanical scan-head. The transducer experimental set-up is shown in Figure 2.2. All experiments were done at a frequency of $25 \mathrm{MHz}$, sending a 30 cycle pulse with increasing pressure. The pressure settings on the VEVO 770 are set at specific values and represented as percentage power. The pressure used are in Table 2.1. The pressures represented in this work were achieved through calibration experiments done in the past by masters thesis student Sara Iradji, in Dr. Kolios' lab [35]. In each experiment, for each percent power setting, 25 data sets were collected, with one data set containing 100 RF lines. One RF line contains the backscatter data for everything in the vertical path of the transducer element as it scans mechanically across the scanhead of the trnasducer. There are 14 different pressure settings used for microbubbles, this includes all of the settings listed in Table 2.1. Thus, $35000 \mathrm{RF}$ lines were saved for each pressure collected. For nanobubbles, 
TABLE 2.1: VEVO 770 Pressure (in MPa) from Percent Power Settings.

\begin{tabular}{lc}
\hline$\%$ Power & Pressure (MPa) \\
3 & 0.075 \\
6 & 0.20 \\
8 & 0.25 \\
10 & 0.30 \\
13 & 0.40 \\
16 & 0.50 \\
20 & 0.60 \\
25 & 0.80 \\
32 & 1.0 \\
40 & 1.2 \\
50 & 1.5 \\
63 & 2.0 \\
79 & 2.4 \\
100 & 3.0 \\
\hline
\end{tabular}

11 pressure settings were used, starting at $0.075 \mathrm{MPa}$ and finishing at $1.5 \mathrm{MPa}$. The total for nanobubbles was 27500 RF lines.

To summarize, there were five microbubble and five nanobubble experiments completed one for each type of bubble shell: Lipids, $+\mathrm{G},+\mathrm{PG},+\mathrm{PGG}$, and + Xlink. The nanobubbles have 11 pressure settings and the microbubbles have 14 pressure settings, with 25 data sets per pressure, and $100 \mathrm{RF}$ lines per data set. All of this was repeated, to ensure reproducibility, for a total of two experiments for each UCA size and shell type. The first experiment for each shell type and size is referred to as Micro \#1 and Nano \#1. The repeat of the same experiment is referred to as Micro \#2 and Nano \#2 That is a grand total of 625 $000 \mathrm{RF}$ lines in which to examine and isolate individually oscillating bubbles. The data collected required sorting, processing and analysis.

\subsubsection{Microbubble Experimental Protocol}

The experimental set up required two beakers that both contain $500 \mathrm{~mL}$ of deionized water. The transducer is placed in one beaker with the scanhead held just above the surface of the water by a clamp. Once this setup has been prepared, $1 \mathrm{~mL}$ of the microbubble 


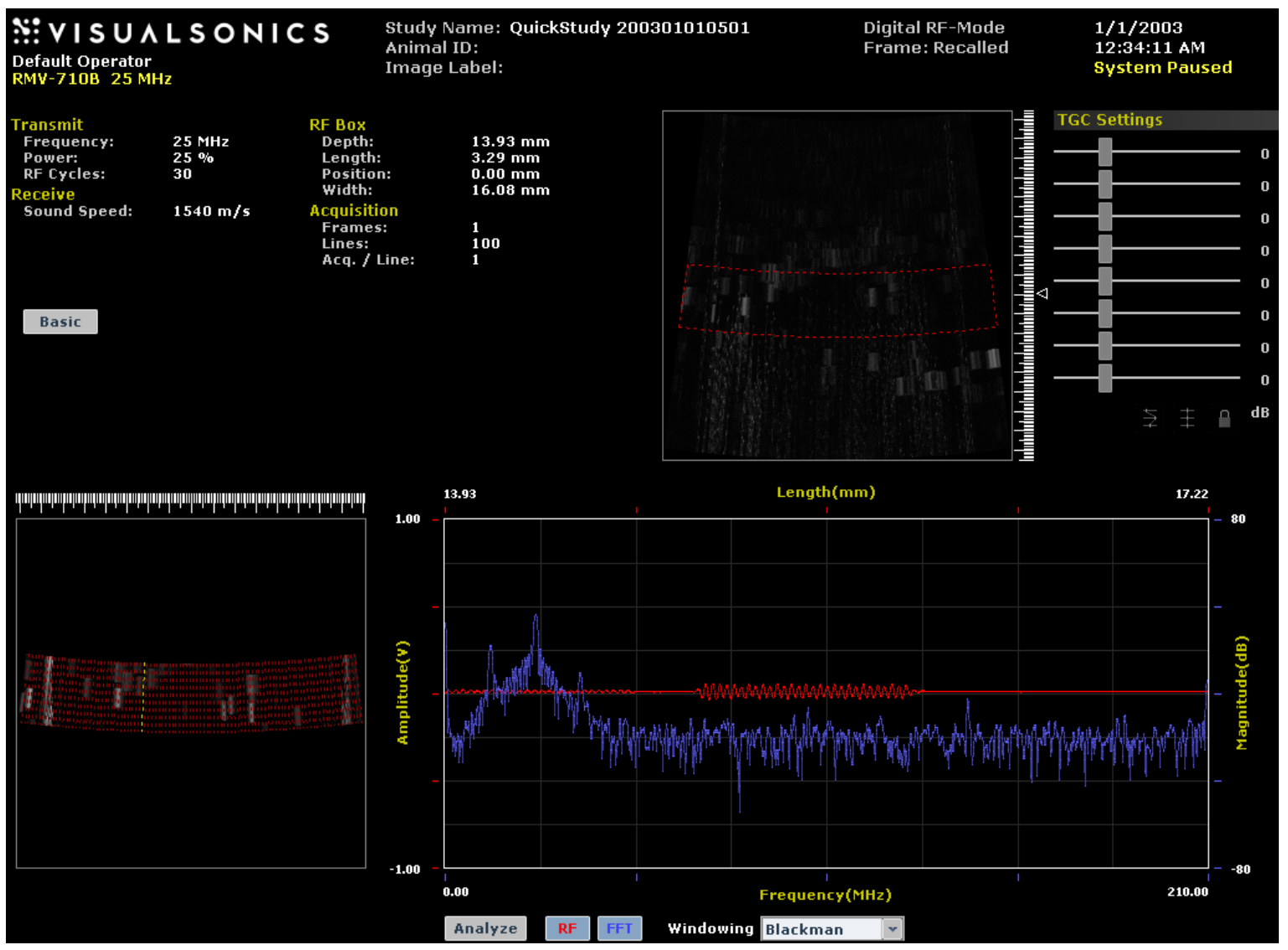

FIGURE 2.3: A screenshot taken during an experiment. The top panel shows the region of data in the dashed red box and each of the small white lines is a bubble undergoing oscillation. The bottom left panel shows the $100 \mathrm{RF}$ lines of data collected of which each one can be selected and the power spectrum and RF line will be displayed in the bottom right panel. 
solution is pipetted into the other beaker which is used as a first dilution of the microbubbles. The beaker is stirred gently with a stirring stick to mix the microbubble sample. Next, a $1 \mathrm{~mL}$ sample is taken of the diluted microbubbles using a pipette and put into the beaker containing the transducer. The beaker is lifted and swirled gently to stir the microbubbles. At this point microbubbles are moving visibly in the top right box on the screen of the VEVO 770. This can be seen in a screenshot taken during an experiment in Figure 2.3. This box of moving bubbles is monitored throughout the experiment to make sure bubbles are always present. Data is collected beginning at the lowest percent pressure available 3\% and increased after capturing 25 data sets or each pressure. Each data set corresponds to one image (Figure 2.3) and $100 \mathrm{RF}$ lines. For the microbubble experiments all percent powers of the VEVO 770 were used in Table 2.1.

\subsubsection{Nanobubble Experimental Protocol}

The nanobubble experiments have the same set up as that of the microbubble experiments. A pipette was used to extract $8 \mu \mathrm{L}$ of nanobubbles to be diluted into the first beaker of DI water which is gently stirred. This is then doubly diluted by pipetting $1 \mathrm{~mL}$ of the bubble solution in the first beaker and placing it into the second beaker with the transducer in it. The beaker is gently swirled, monitoring the screen for the presence of bubbles. Additional $1 \mathrm{~mL}$ amounts are pipetted from the first to second beaker if bubbles are not visually present. This was additionally required between 4-12 times during the nanobubble experiments whereas for the microbubble experiments it was only required one additional time. Measurements were taken beginning at the lowest pressure 0.075 MPa and increased for each setting (Table 2.1) after capturing 25 data sets at each pressure. For the nanobubble experiments bubble destruction was observed at $32 \%$ power $(1.0 \mathrm{MPa})$ so the range of these experiments was capped at $50 \%$ power (1.5 MPa). 


\subsection{Algorithm for Signal Selection}

Isolation and selection of single bubble signals was required to study the bubble backscatter. To most rigorously compare signals from different bubble shell types, only RF lines that contained a single bubble's oscillation were included in the data. Signals that contained more than one bubble, no bubbles and bubbles whose signals were truncated are all removed through the analysis code written and run using Matlab. An example of an accepted bubbles' RF line is shown in Figure 2.4.

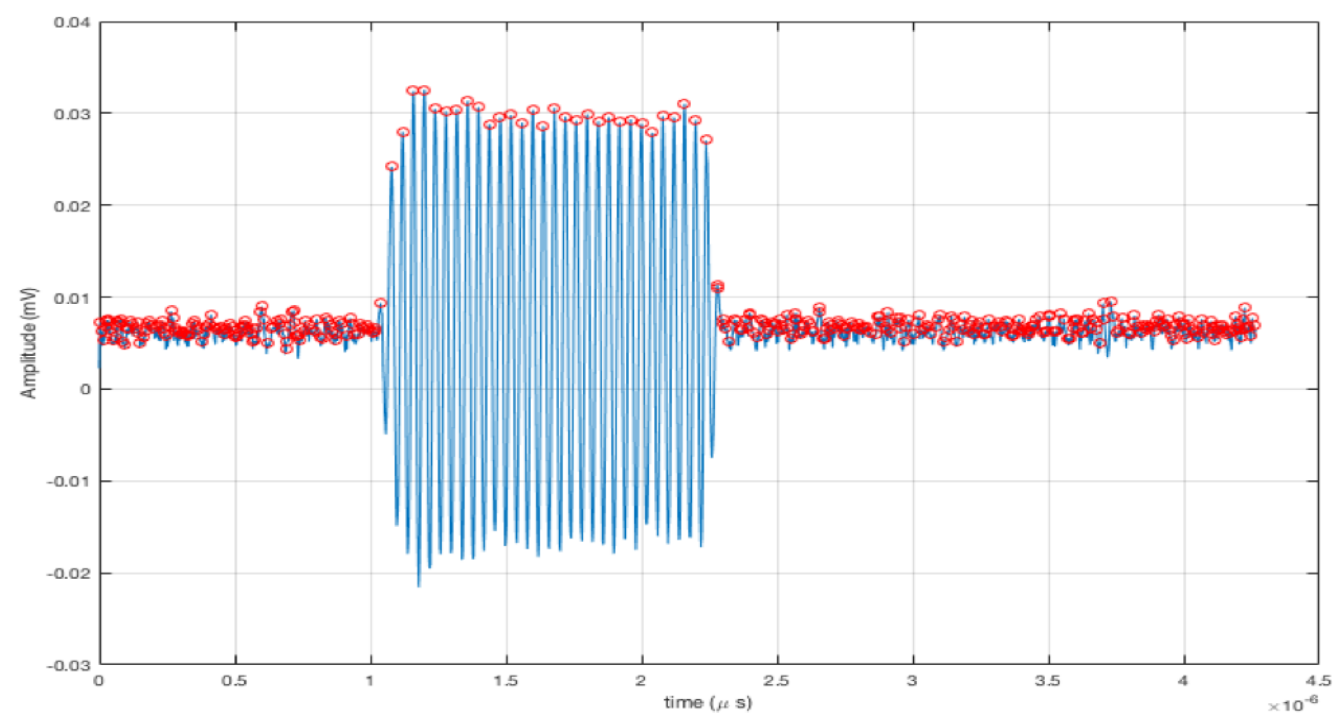

FIGURE 2.4: This is an example of the RF line of a bubble that was accepted based on our algorithm. The red circles are highlighting each peak detected.

Initially the process of single bubble isolation was labour intensive, requiring the viewing of each RF line to establish whether it met our criteria. A large part of this work was in developing the code that made the analysis of this work achievable in a reasonable amount of time. 


\subsubsection{Criteria}

This section will describe the Matlab code that automates the individual bubble selection process. The code must be run for each percent power in each experiment separately, selecting and using the appropriate naming convention for all bubbles found. Initially the code loads each data set and each of the 100 RF lines within it. The power spectrum is calculated by finding the fast fourier transform of each RF line. A signal is kept at this stage if its max peak of its power spectrum is above $0 \mathrm{~dB}$, essentially an indication as to whether there are any bubbles present in that RF line. If the signal's max power spectrum falls below $0 \mathrm{~dB}$ the signal is removed. This is shown in Figure 2.5. This is the initial criteria of acceptance to assist in eliminating significant amounts of data that don't need further analysis. For the lower pressures this step removes approximately $60 \%$ of RF lines and for the higher pressures this removes approximately $30-40 \%$ of RF lines.

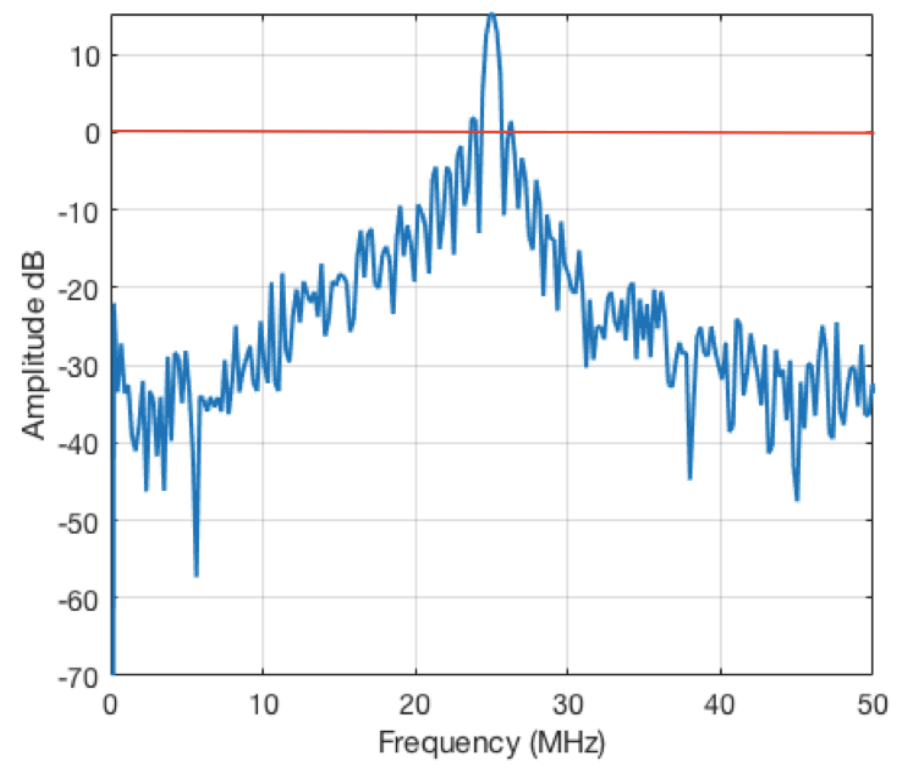

FIGURE 2.5: All RF lines with a max power spectrum below $0 \mathrm{~dB}$ are removed

Next, the RF lines are examined individually. In each of the remaining RF lines all of the peaks are located using a Matlab function. The maximum peak is located and a bubble threshold of $50 \%$ of the max peak is defined. The threshold of $50 \%$ was decided by comparing this algorithm with the manual method of selecting signals to ensure the largest 
number of signals was being accepted. The truncated signals are removed by ensuring that the first and second peaks, as well as the last and second last peaks found in the RF line, fall below the bubble threshold of $50 \%$ of the max peak of that RF line. If they do not fall below this threshold then they are truncated signals and are removed. An example of a truncated signal is shown in Figure 2.6.

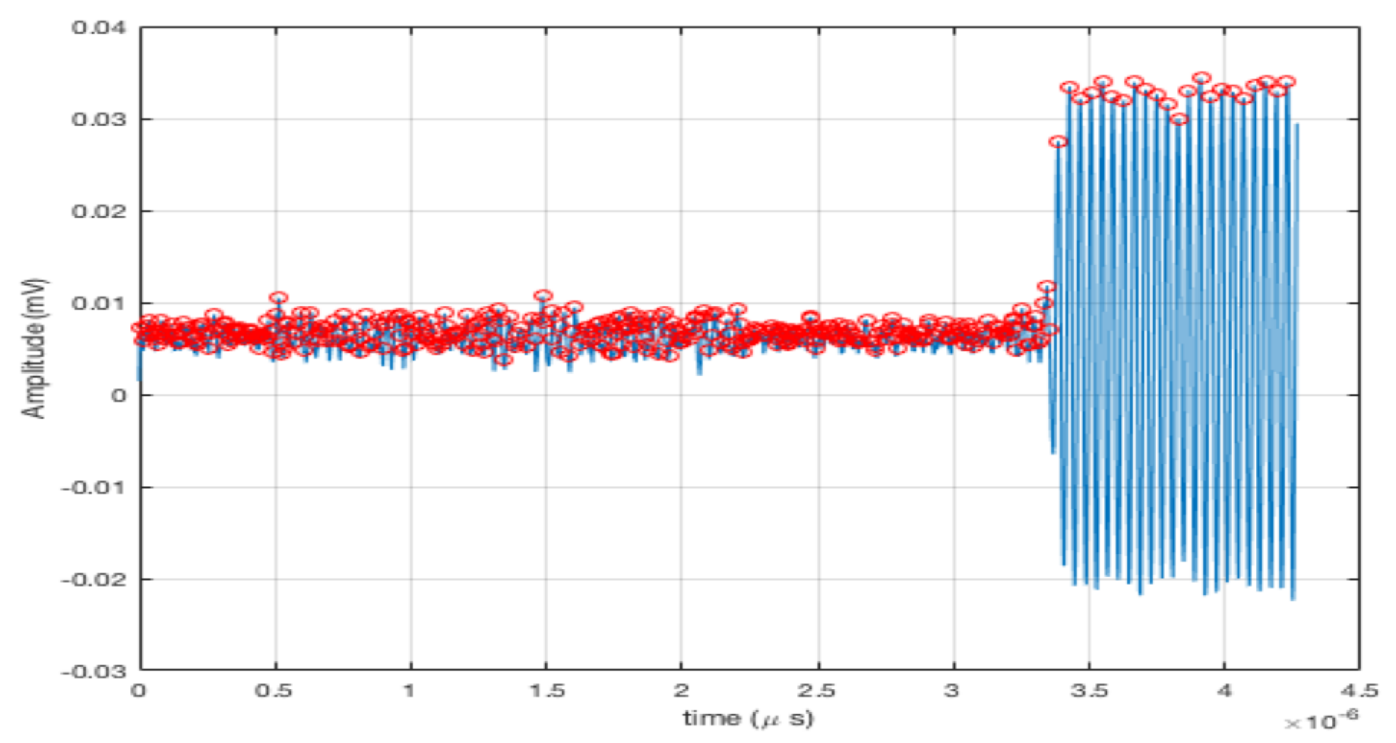

FIGURE 2.6: This is an example of an RF line of a truncated bubble. The last and second last peaks highlighted by the red circles are well over $50 \%$ of the maximum peak of the RF line. This RF line would be removed using our algorithm.

Next, the number of peaks above $50 \%$ of the max peak are counted, the acceptable range falls between 1-32 peaks, since a 30 cycle pulse is sent. If the RF line has more than 32 peaks above $50 \%$ of the max peak it is assumed to be excessive noise or more likely more than one bubble detected and is thus removed. An example of an RF line with more than one bubble in it is shown in Figure 2.7.

The last step of the criteria is to ensure that there are no other bubbles are within the RF line. This is done by creating a peak to peak noise threshold for the non-bubble portion of the RF line from the noise threshold for each particular experiment. If this non-bubble portion of the signal has any peak to peak amplitude that is greater than the established noise threshold then a second bubble is assumed to be present and the RF line is discarded. 


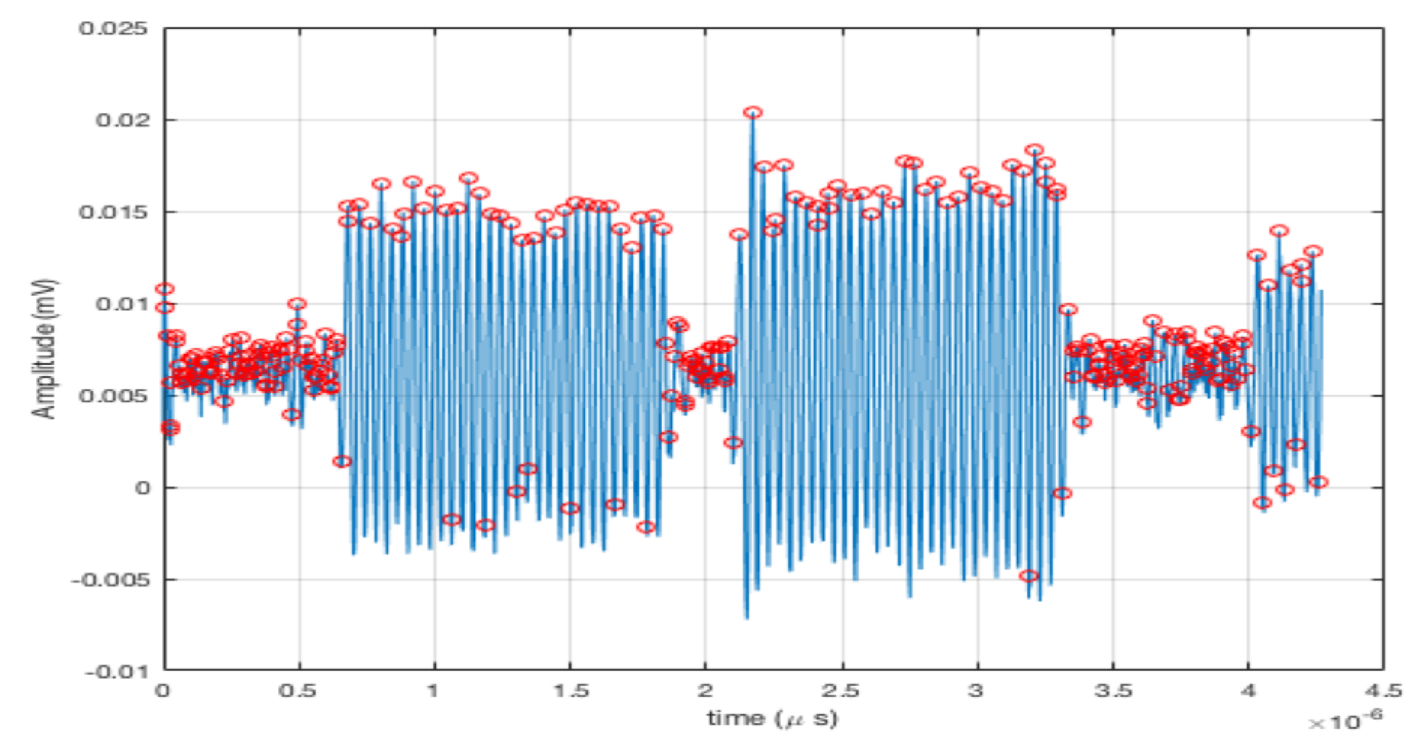

FIGURE 2.7: This is an example of an RF line that contains more than one bubble in it. This RF line would be removed using our algorithm.

If the peak to peak amplitude falls below the threshold then the RF line is accepted and the bubble is included in the analysis. This step is done by first loading all RF lines for a particular pressure exposure ( 25 data sets) that have a maximum noise spectrum that falls below $-10 \mathrm{~dB}$. The threshold of $-10 \mathrm{~dB}$ was chosen because it is a cut off of which below there were no bubbles present in these signals, only noise. Figure 2.8 shows all the RF lines within one pressure that pass this criteria. This is the base noise level.

All RF lines are then enveloped. A sample of the envelope for a single RF line is shown in Figure 2.9. The average peak to peak distance of the envelope is calculated for each RF line and then the average is calculated across all RF lines.

This peak to peak distance is multiplied by 2.5, a number that was determined by deciding what was above the noise level. This is the noise threshold. Outside of the 30 cycle bubble signal, the RF lines must have a max peak to peak distance that is below the noise threshold. All signals with RF lines that don't follow this criteria are rejected.

The signals that have passed all of the criteria are displayed to be viewed and named. A sample of how this appears during the running of the algorithm using Matlab is 


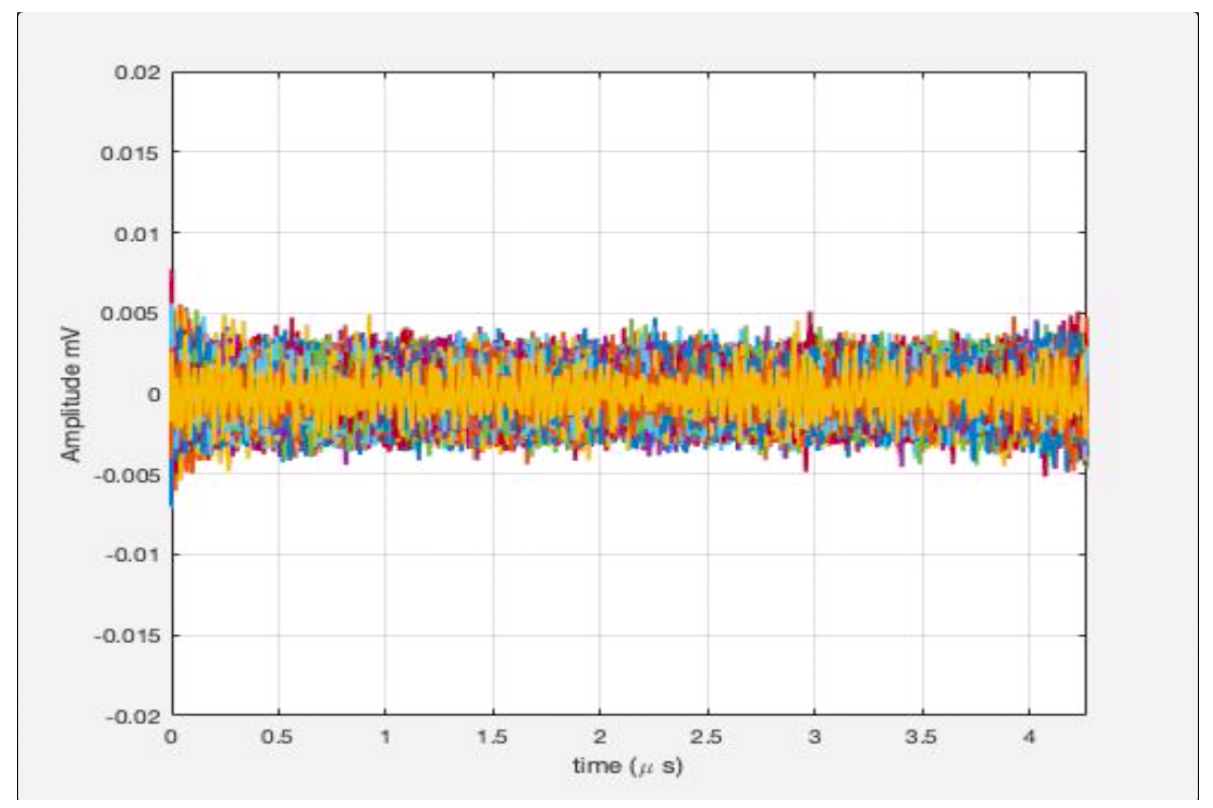

FIGURE 2.8: All RF lines layered on top of one another within one pressure exposure setting ( 25 data sets) that fall below a maximum noise spectrum of $-10 \mathrm{~dB}$ (average peak to peak amplitude of below $0.01 \mathrm{mV}$ in the RF line). This representative data is from microbubble experiment \#1, PGG bubbles at 800 $\mathrm{kPa}$.

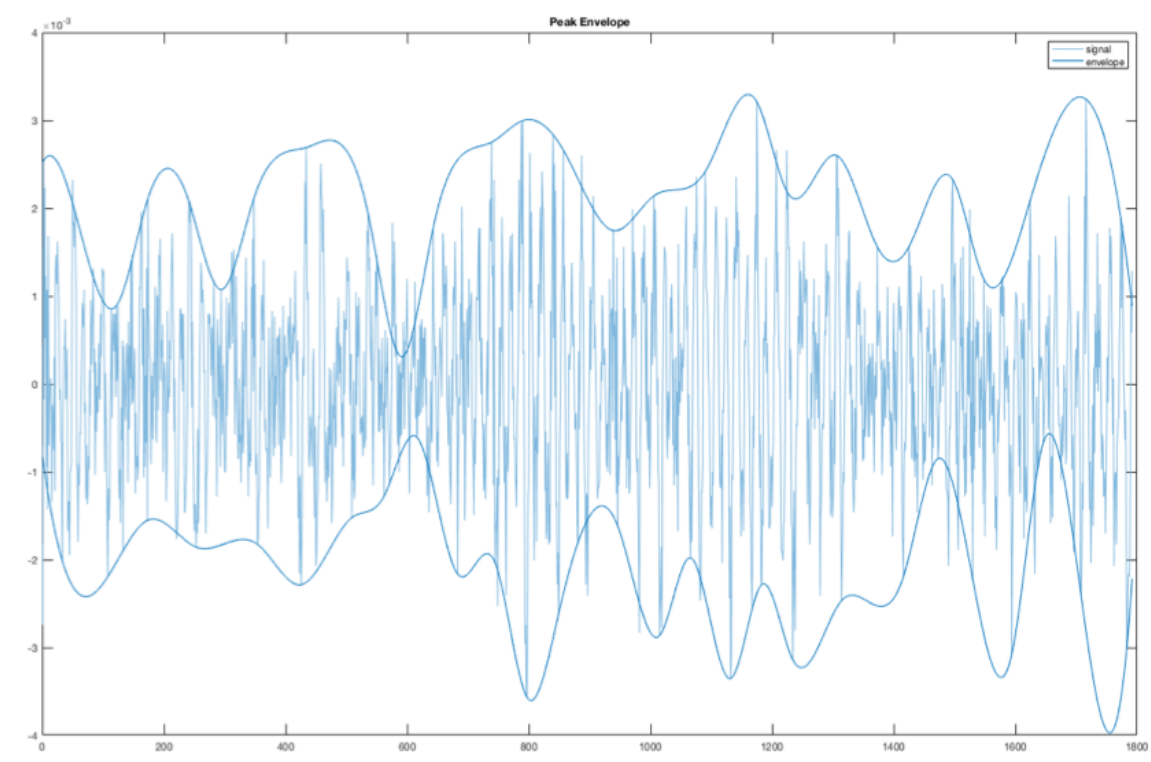

FIGURE 2.9: This demonstrates how the envelope of the RF line appears for one RF line in the process of determining the noise threshold. 

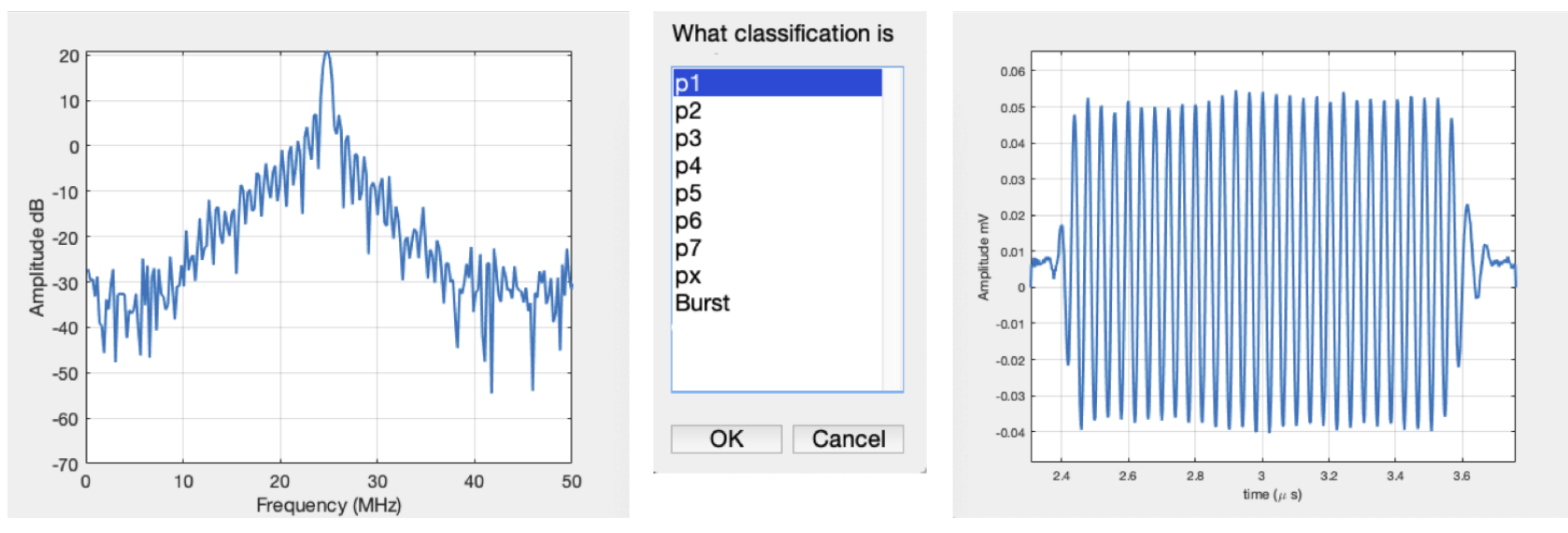

FIGURE 2.10: An example of the classification part of the algorithm for signal selection as it appears when running the code. The power spectrum and RF line are displayed for inspection and naming classification.

displayed in Figure 2.10.

\subsection{Signal Classification and Naming Procedure}

This section explains how the signals are named based on characteristics derived from their power spectrum and RF line. It also provides figures that show representative signals of the different types of oscillations observed in this work.

\subsubsection{The Classification of Accepted Signals}

\section{Linear Accepted Signals}

The signals fall under two main categories: linear and non-linear. A linear oscillation has a minimum and maximum radius that is consistent throughout the entire oscillation. The linear signals are called period 1 oscillations or $\mathrm{p} 1$ for short. An example of p1 signals can be seen in Figure 2.11. There are varying types of these oscillations shown, some for which there are no significant variations of signal amplitude for the entire 30 cycle 
exposure (A), and some for which a steady state is reached after an initial transient response $(B, C)$.
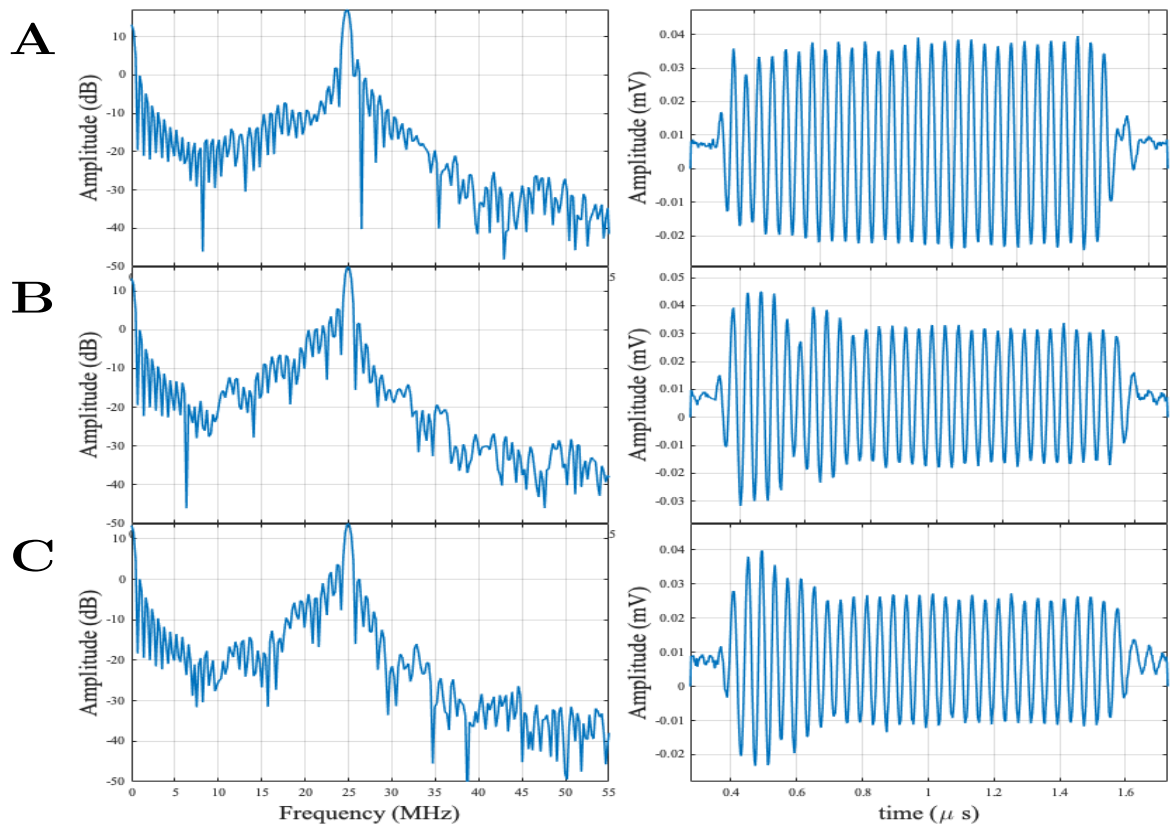

FIgURE 2.11: Period 1 (p1) oscillation examples. A shows a steady p1 signal, B shows a signal that starts with some transient behaviour but then quickly settles in to a linear oscillation. $\mathrm{C}$ shows a signal in which the bubble might have lost air during the first few cycles of oscillation resulting in a smaller peak to peak amplitude in the remaining cycles of the signal.

\section{Non-linear Accepted Signals}

The non-linear signals deviate from this pattern. In some non-linear signals their pattern is straight forward to classify as they exhibit consistent features and named as period 2, period 3, period 4, period 5, period 6 and period 7 depending on the pattern observed. Signals that demonstrated repetitive patterns beyond period 7 (for example period 8 and period 9) could not be observed because they would not repeat enough times within the 30 cycles to prove reliability of that signal type. The signal was classified based off of the most commonly occurring repetitive sequence in the RF line. 

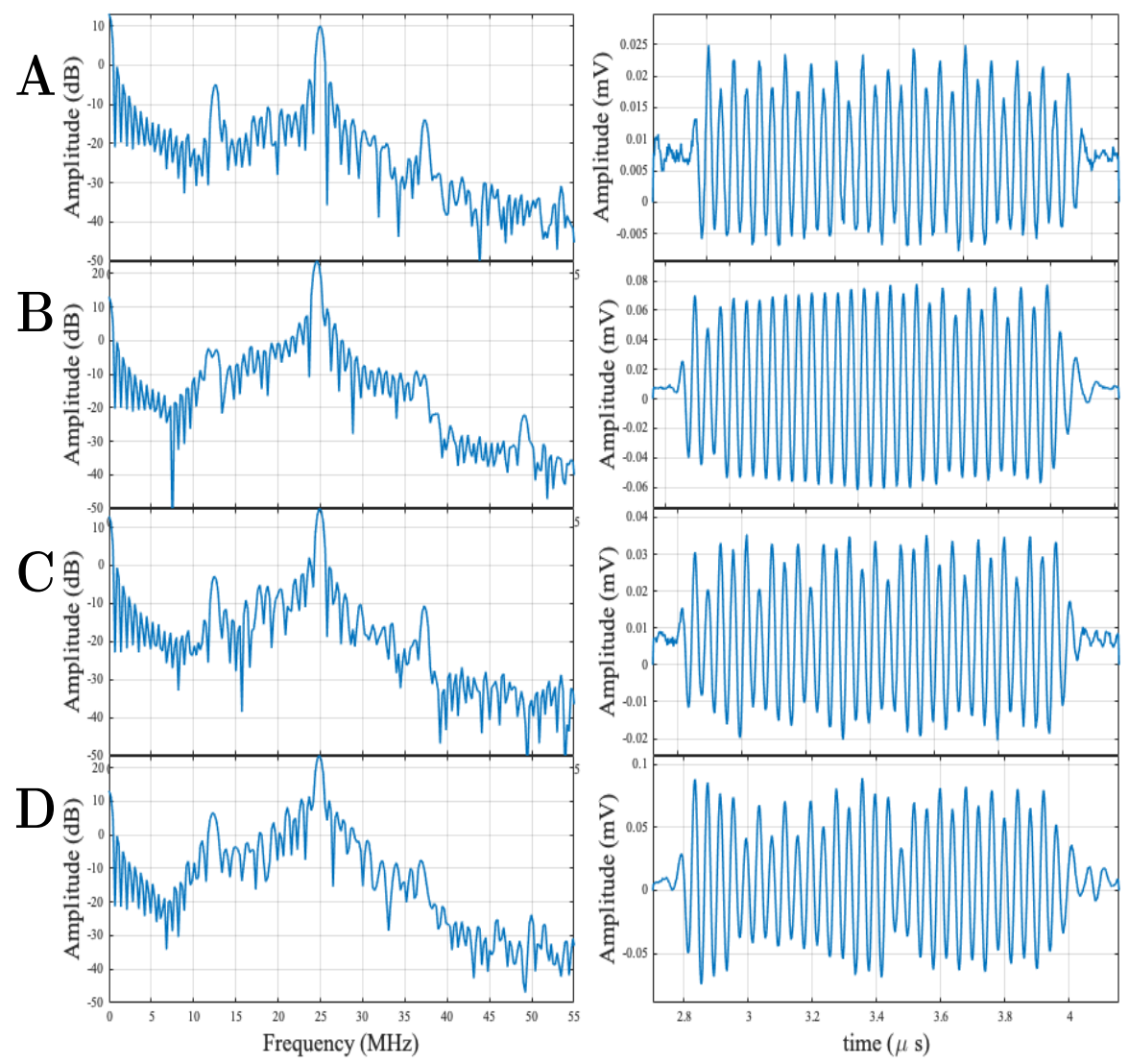

FIgURE 2.12: Period 2 (p2) oscillation examples. A shows a consistent p2 signal for the entire 30 cycle excitation, $\mathbf{B}$ shows a signal that turns into a p2 signal in the last third of the excitation, $\mathbf{C}$ shows a signal that has some $\mathrm{p} 4$ characteristics but is not consistently a p4 signal but is most consistently a p2 oscillation, $\mathbf{D}$ also has some variability in the RF line but is most dominately a p2 signal. 
The signals are classified based on their repetitive patterns. This is easier to see in the RF line but can also be observed in the power spectrum in the peaks and their locations. Figure 2.12 shows some different examples of period 2 (p2) oscillations and how they can vary within the $\mathrm{p} 2$ classification. Most $\mathrm{p} 2$ signals will show prominent peaks in the power spectrum at $\frac{1}{2}$ and $\frac{3}{2}$ the centre frequency, which is $25 \mathrm{MHz}$. In the RF line, the p2 signal is denoted by the alternating heights in its peaks. Every other peak will have a lower amplitude which repeats through a reliable pattern. This allows a grouping and repetition of two peaks throughout the signal.
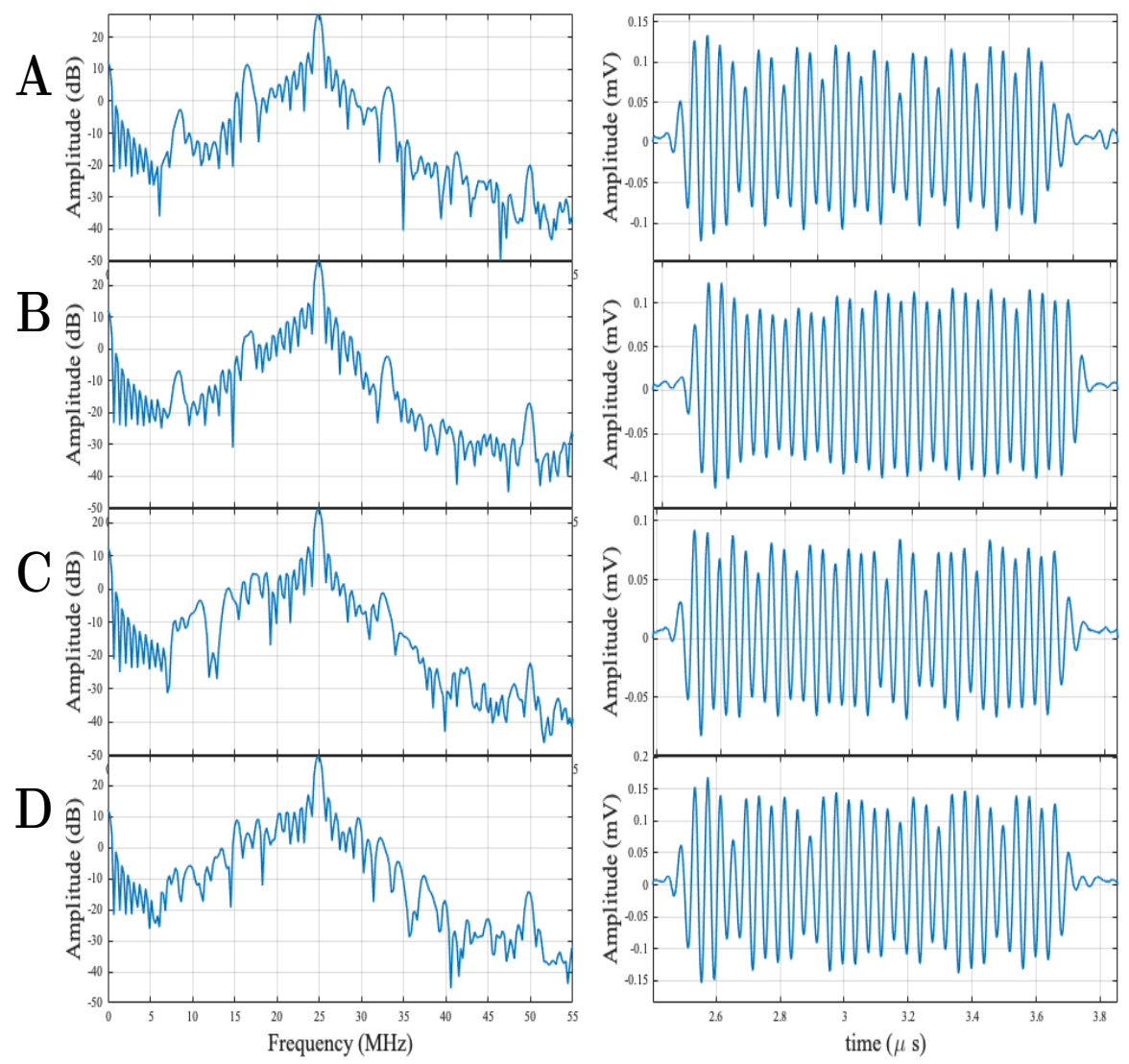

FIGURE 2.13: Period 3 (p3) oscillation examples. A shows a consistent p3 signal in which the pattern of the three different peaks can be seen repeating throughout the oscillation, $\mathbf{B}$ this signal is similar to $A$ but takes slightly longer to adopt its' p3 oscillation, $\mathbf{C}$ this signal shows some occurrence of a p4 oscillation but the $\mathrm{p} 3$ oscillation dominates the signals, $\mathbf{D}$ this shows even more variability but still the most dominant oscillation type is $\mathrm{p} 3$.

The next non-linear oscillation type, ascending in numerical order are the period 
3 (p3) signals. Samples of p3 signals can be seen in Figure 2.13. Similar to the pattern described in $\mathrm{p} 2$ signals in that for the $\mathrm{p} 3$ signals three significant peaks can be seen in the power spectrum (including the centre frequency) and there is a repetitive pattern of three peaks in the RF line.
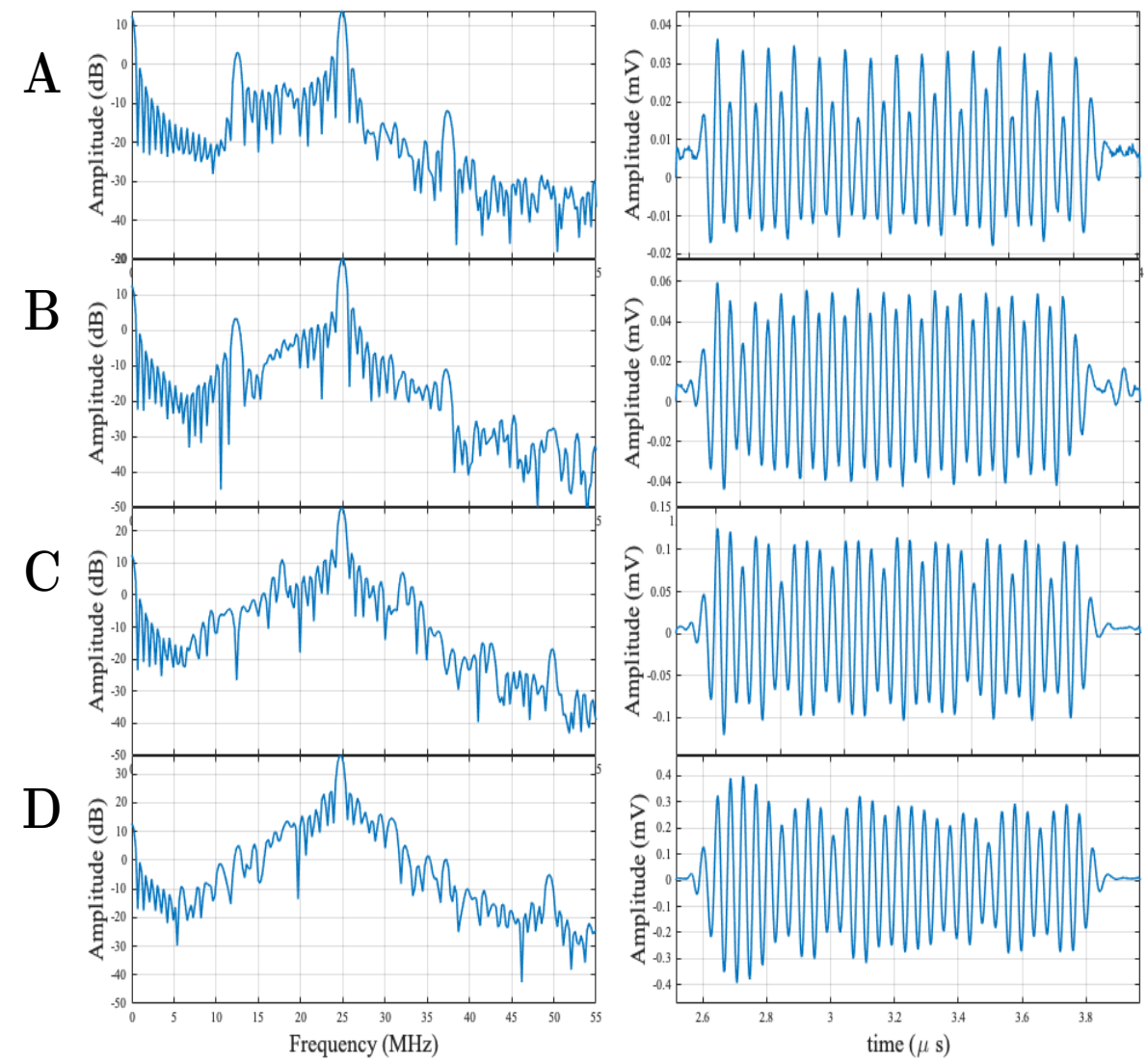

FIGURE 2.14: Period 4 (p4) oscillation examples. A shows a consistent $\mathrm{p} 4$ oscillation that looks similar to p2 but upon closer examination a pattern of 4 emerges, $\mathbf{B}$ shows a p4 signal that begins as a p2 oscillation and then midway through becomes a p4 oscillation, $\mathrm{C}$ shows a different type of $\mathrm{p} 4$ oscillation. This one looks less like a 2 , instead it has clear groups of 4 peaks descending in amplitude throughout. It has some p3 oscillations as well. D shows a signal with a dominate $\mathrm{p} 4$ presents among other non-linear behaviour.

The next consecutive non-linear signal type is period $4(\mathrm{p} 4)$. This oscillations type can look similar to a p2 signal. However, with closer examination a repetitive pattern of 4 peaks in the RF line become clear. Figure 2.14 shows samples of varying types of 44 signals. The power spectrum for the $\mathrm{p} 4$ signals will often contain large peaks in the same positions 
as the p2 signals (at $\frac{1}{2}$ and $\frac{3}{2}$ the centre frequency). P4 signals will also have peaks between those peaks and the centre frequency peak.
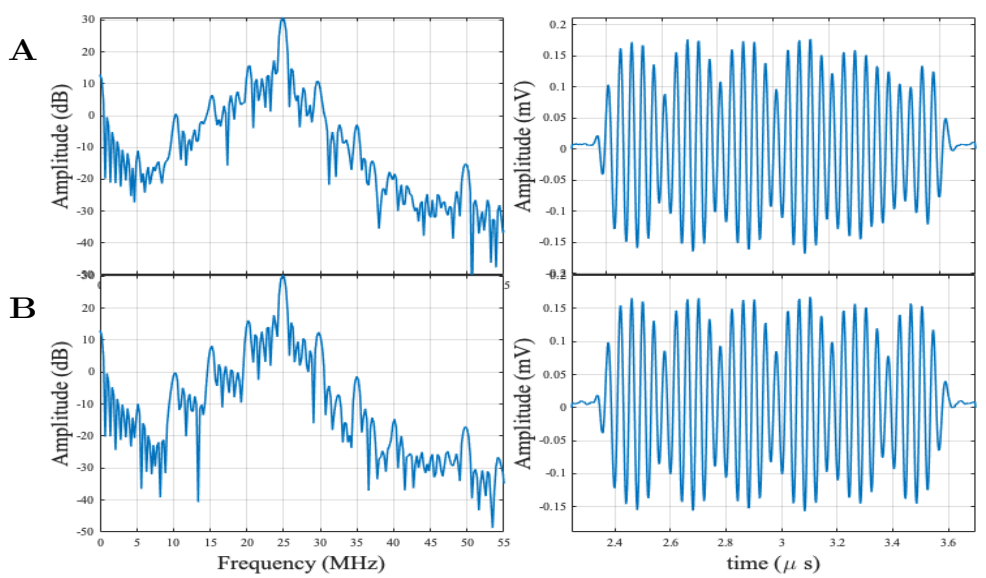

FIGURE 2.15: Period 5 (p5) oscillation examples. A and B shows similar signals demonstrating p5 oscillations, with groups of 5 peaks that repeat throughout the signal.

The last three numbered classifications used in this work are more rare but still observed. This includes p5 which can be seen in Figure 2.15, p6 which is shown in Figure 2.16 and p7 of which there is only one example in Figure 2.17. As with the previously described examples, the number of peaks in a repeated sequence correspond to the classification name and can be observed in the RF line of each example. As the number of peaks in the repeated sequence get larger the capacity to repeat the sequence more than once becomes more challenging. This is why in this work we did not classify signals beyond period 7 considering only 30 cycles were used in this work.

For some signals, there is no obvious pattern in oscillation. There is evident nonlinearity due to the different heights in peaks of RF lines however there is not a reliable, repeatable sequence. These signals have been denoted px and examples are shown in Figure 2.18. 

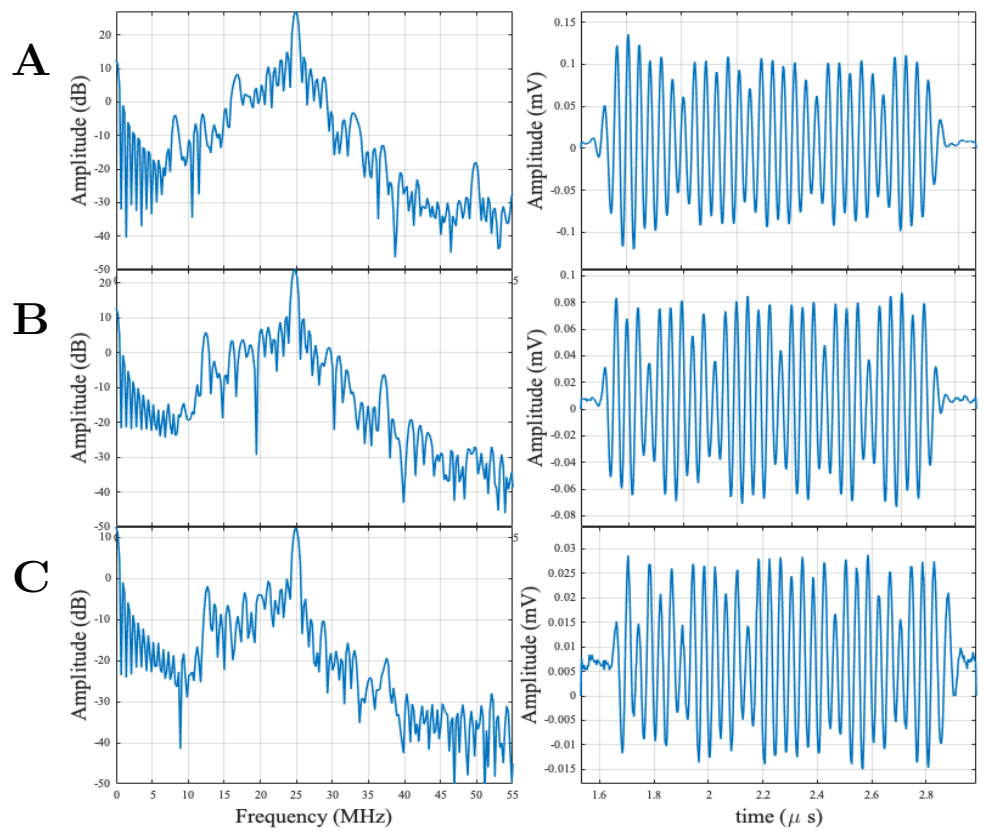

FIgURE 2.16: Period 6 (p6) oscillation examples. A shows a p6 signal that looks similar to a $\mathrm{p} 3$ but has a repeated pattern of groups of 6 peaks. B shows the most dominate occurrence of $\mathrm{p} 6$ oscillation with some $\mathrm{p} 4$ oscillations in this signal. C shows a signal with the only recurring oscillation is p6.
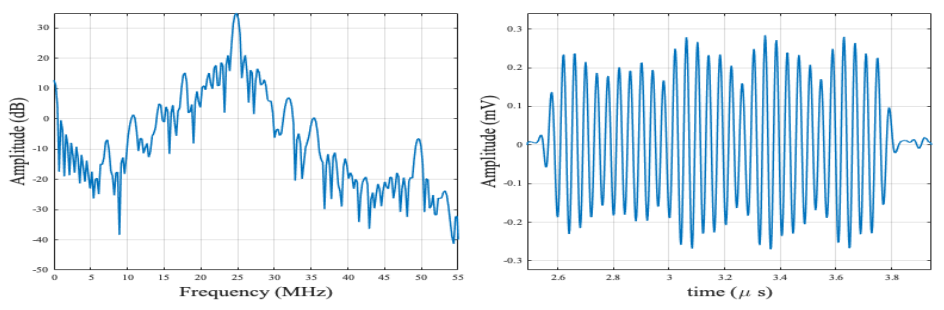

FIGURE 2.17: Period 7 (p7) oscillation example. Two groups of 7 peaks are in this signal. 

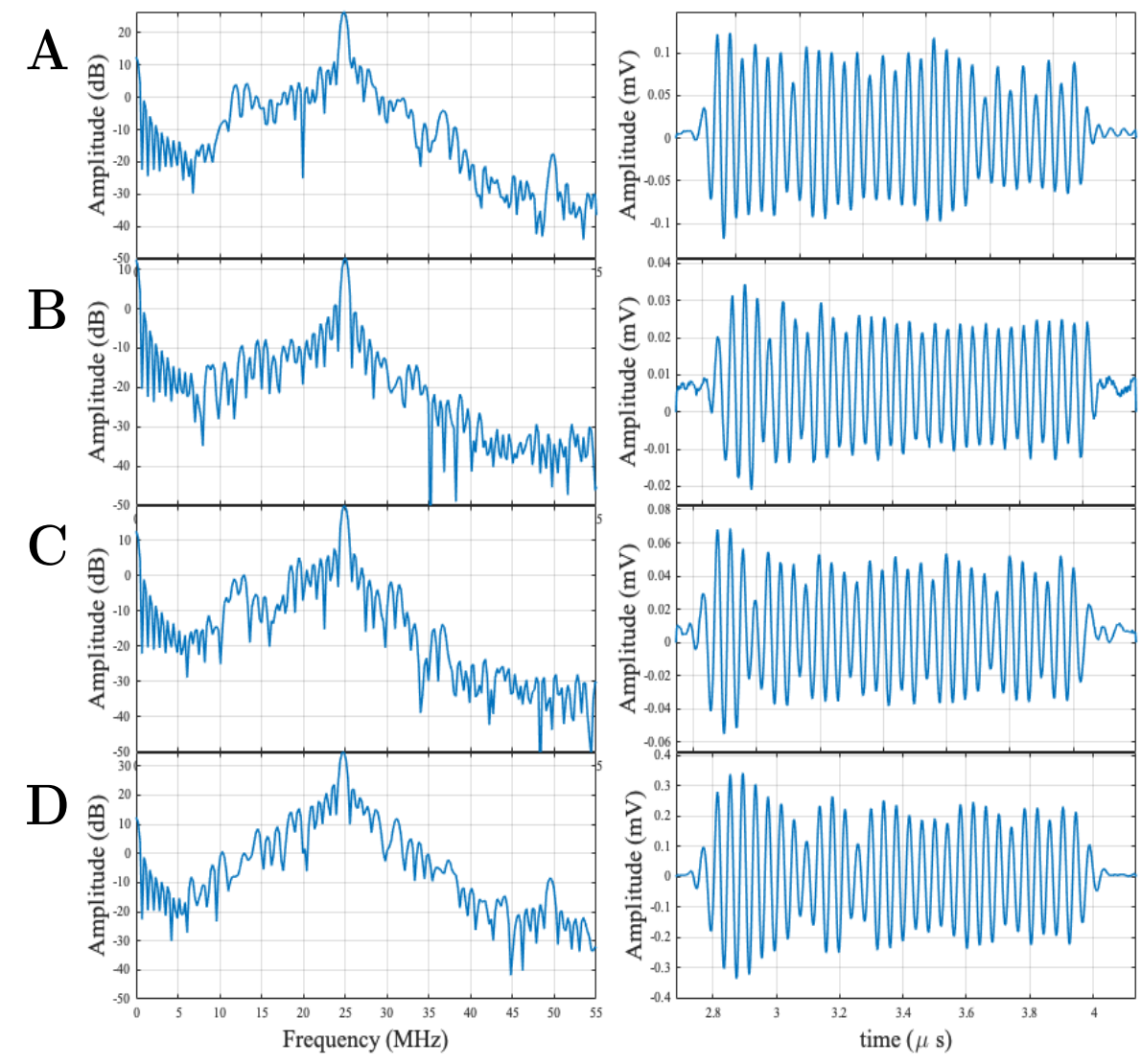

FIGURE 2.18: Non-linear oscillations that cannot be easily classified are called px signals. 


\subsubsection{Rejected Signals}

Some signals that pass all the criteria within the algorithm described in section 2.4 are still rejected in the classification process. This could be for one of two reasons. The most common reason a signal is rejected during the naming process is that it clearly demonstrates a bubble that does not complete all 30 oscillations and thus, bursts. This can be seen in Figure 2.19. These signals were classified as Burst and analyzed separately. The code is purposefully designed to show all signals that include a bubble undergoing at least one cycle of oscillation so that the burst signals would be included.
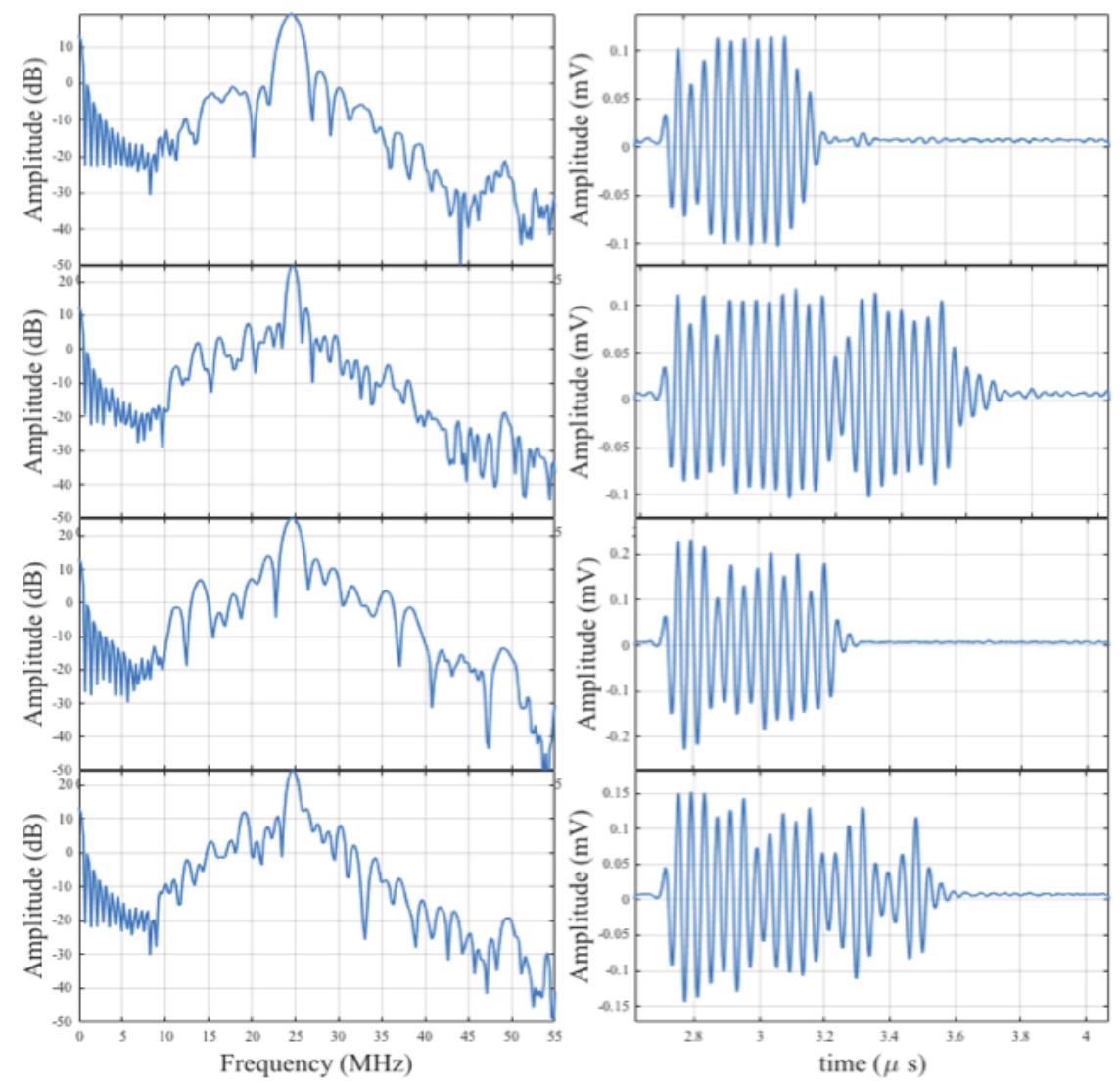

FIGURE 2.19: Examples of bubbles that burst before completing 30 oscillations.

Another reason that a signal may be rejected at the classification section of the algorithm is the interference of another bubble within the gated bubble signal. This signal 
was not observed to have more than one bubble during the algorithm and therefore was not rejected. This is largely due to the requirement to set a gate around the 30 cycle bubble oscillation which for simplicity during coding and optimal visualization extends slightly beyond the 30 cycles and can thus sometimes contain more than one bubble within it without the algorithm detecting it.

\subsubsection{Analysis of Classified Signals}

The first step in the analysis was on the initial threshold in our algorithm which looks at the percentage of the total signals that are considered once signals with no bubbles present have been removed.

Once the signals have been isolated, the classified signals are analyzed further through multiple methods. In our analysis we examine the total number of signals that are classified into linear, non-linear and burst signals. We also consider each of these subcategories as a percentage of occurrence. Furthermore, we investigated the prevalence of the different types of non-linear oscillations (p2, p3, p4, etc.). Finally, we analyzed the amplitudes of oscillation of the fundamental, subharmonic and ultra harmonic frequencies. 


\section{Chapter 3}

\section{Results}

\subsection{Size Measurements}

\subsubsection{Microbubbles}

TABLE 3.1: Microbubble experimentally measured diameters and standard deviation.

\begin{tabular}{lcc} 
Shell & \multicolumn{2}{c}{ Diameter $(\mu \mathrm{m})$} \\
\hline & Micro \#1 & Micro \#2 \\
\hline Lipid & $1.5 \pm 0.65$ & $1.4 \pm 0.74$ \\
PG & $1.7 \pm 0.80$ & $1.6 \pm 0.72$ \\
G & $1.5 \pm 0.56$ & $1.6 \pm 0.72$ \\
PGG & $1.1 \pm 0.75$ & $1.6 \pm 0.70$ \\
Xlink & $1.8 \pm 0.63$ & $1.8 \pm 0.69$ \\
\hline
\end{tabular}

The measurements that were done with the Beckman Coulter containing the mean diameter with standard deviation for the microbubble experiments are given in table 3.1. The size distribution graphs are included in Appendix A.

\subsubsection{Nanobubbles}

Table 3.2 shows the mean sizes and standard deviations for the diameter measurements of the nanobubbles. These measurements were completed using Archimedes and 
TABLE 3.2: Nanobubble experimentally measured diameters and standard deviation.

\begin{tabular}{lcc} 
Shell & \multicolumn{2}{c}{ Diameter $(\mu \mathrm{m})$} \\
\hline & Nano \#1 & Nano \#2 \\
\hline Lipid & $0.17 \pm 0.06$ & $0.39 \pm 0.11$ \\
PG & $0.19 \pm 0.10$ & $0.26 \pm 0.15$ \\
G & $0.19 \pm 0.11$ & $0.27 \pm 0.12$ \\
PGG & $0.25 \pm 0.12$ & $0.37 \pm 0.11$ \\
Xlink & $0.23 \pm 0.10$ & $0.21 \pm 0.15$ \\
\hline
\end{tabular}

they show the sizes for buoyant and non-buoyant particles. The size distribution graphs are included in Appendix A.

\subsection{Signal Count Statistics}

\subsubsection{Initial Criteria Threshold}

In presenting the first results, it is important to note that the counts represent the total sum of valid measurements within a specific category. The number of classified bubbles does provide a baseline measurement showing the minimum total responses from which the data is compiled. Figure 3.1 shows the total accepted signal count for each type of shell and each pressure collected for the microbubble experiments and Figure 3.2 shows the same data for the nanobubble experiments. The smallest number of signals accepted is 15 for the nanobubbles and 29 for the microbubbles. The graphs show the lowest number of signals recorded for a specific category. The bubble shell type is provided in the graph caption.

Figure 3.3 demonstrates the percentage of total signals for microbubbles that pass the initial criteria explained in section 2.4.1 (as opposed to the absolute number in 3.1). Figure 3.4 shows the percentage of signals that pass the initial criteria for nanobubbles. The colour represents each type of bubble shell. Each percentage shown for each pressure (percent power) for each bubble shell type is out of a total number of 2500 RF lines, therefore, each $1 \%$ represents $25 \mathrm{RF}$ lines (signals). 


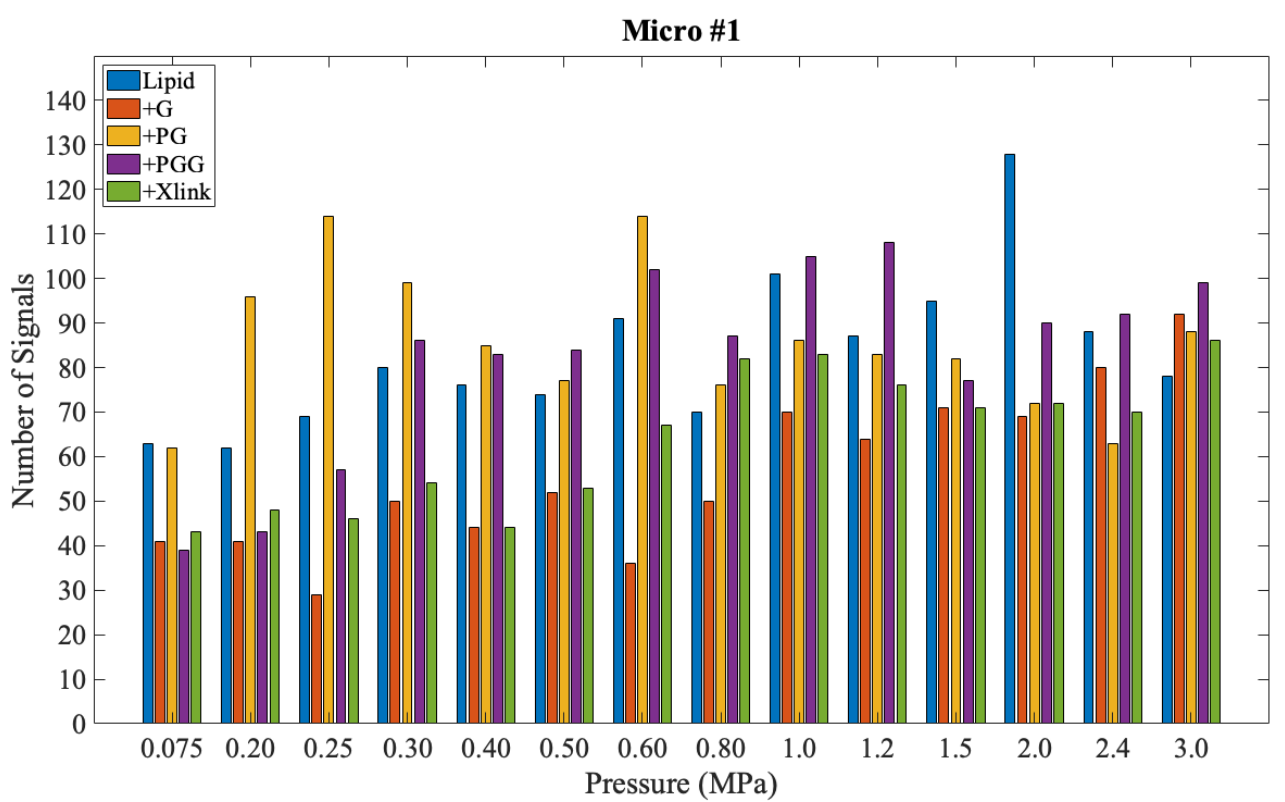

(A) 29 signals ( $G$ at $8 \%$ power)

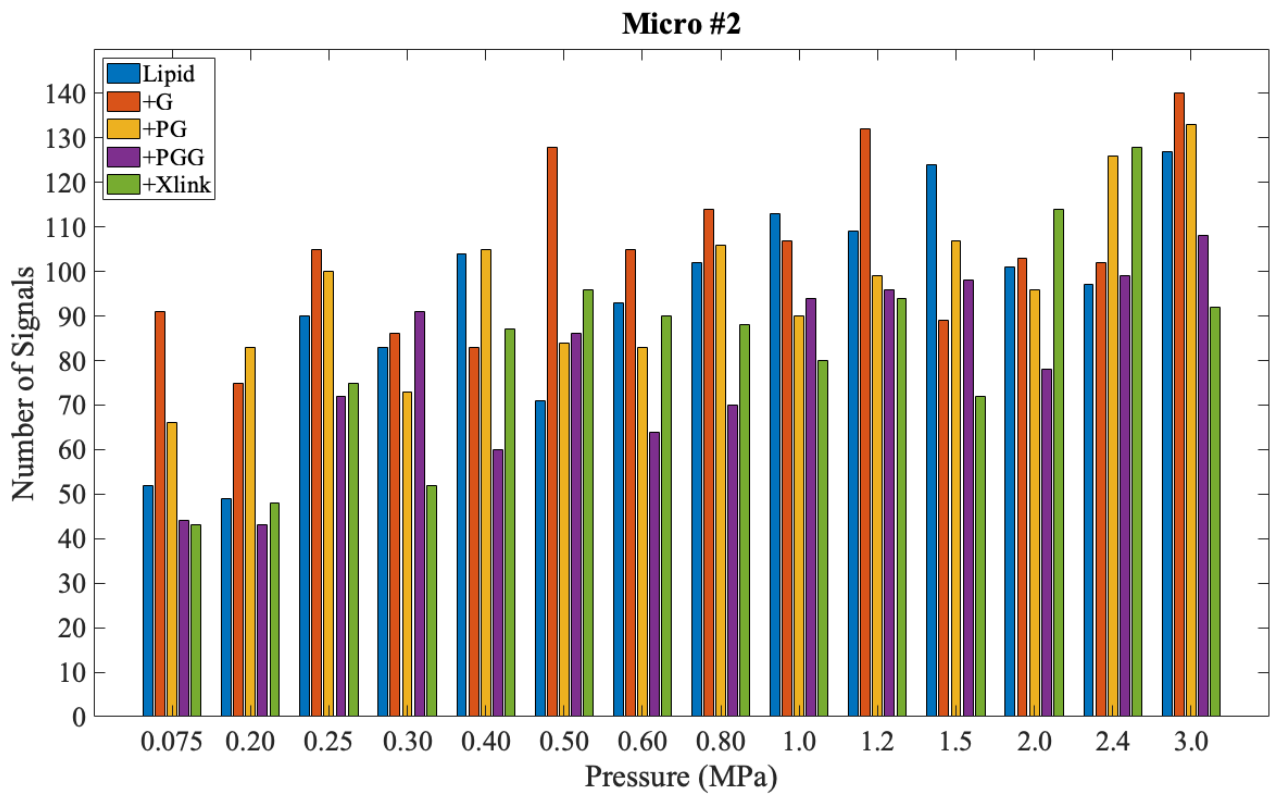

(B) 43 signals (Xlink at 3\% power and PGG at 6\% power)

FIGURE 3.1: Total number of classified bubbles across pressure for both of the microbubble experiments. The sub-caption below each graph shows the lowest number of classified signals for each experiment. 


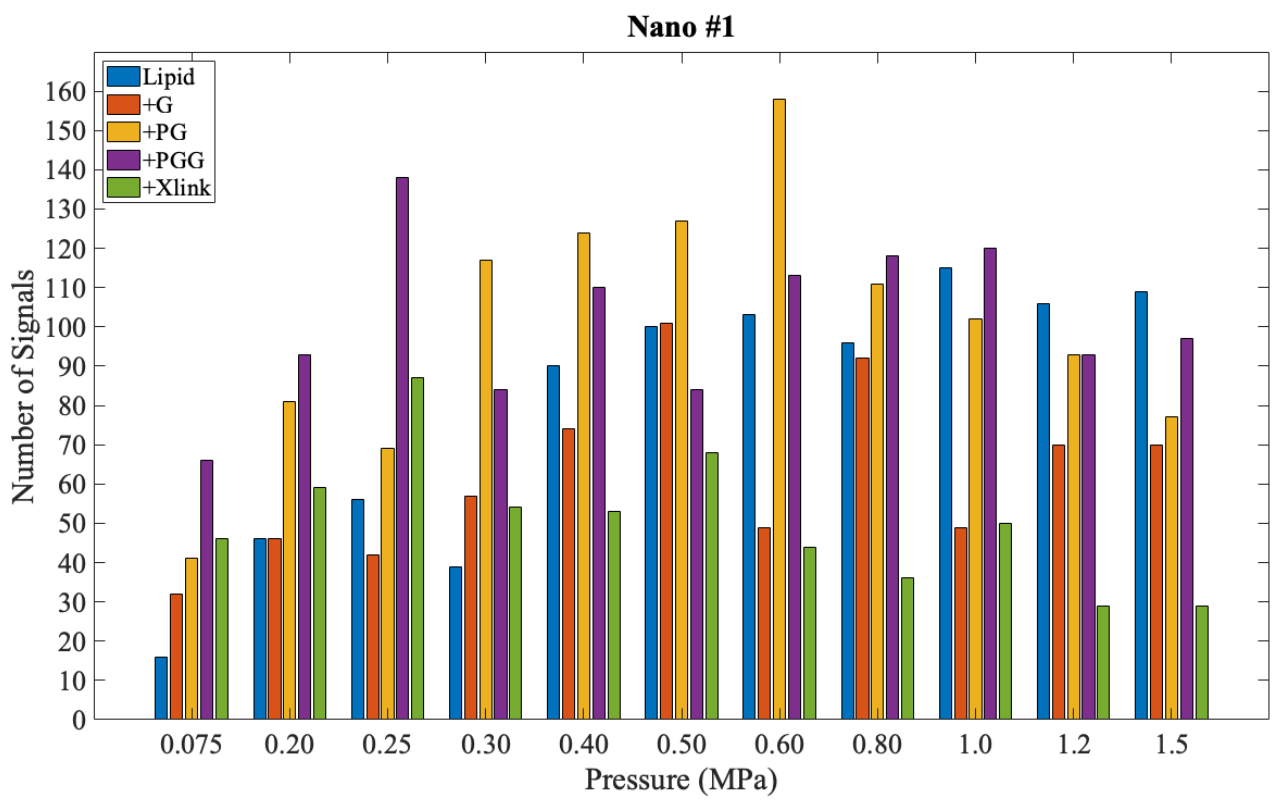

(A) 16 signals (lipid at $3 \%$ power)

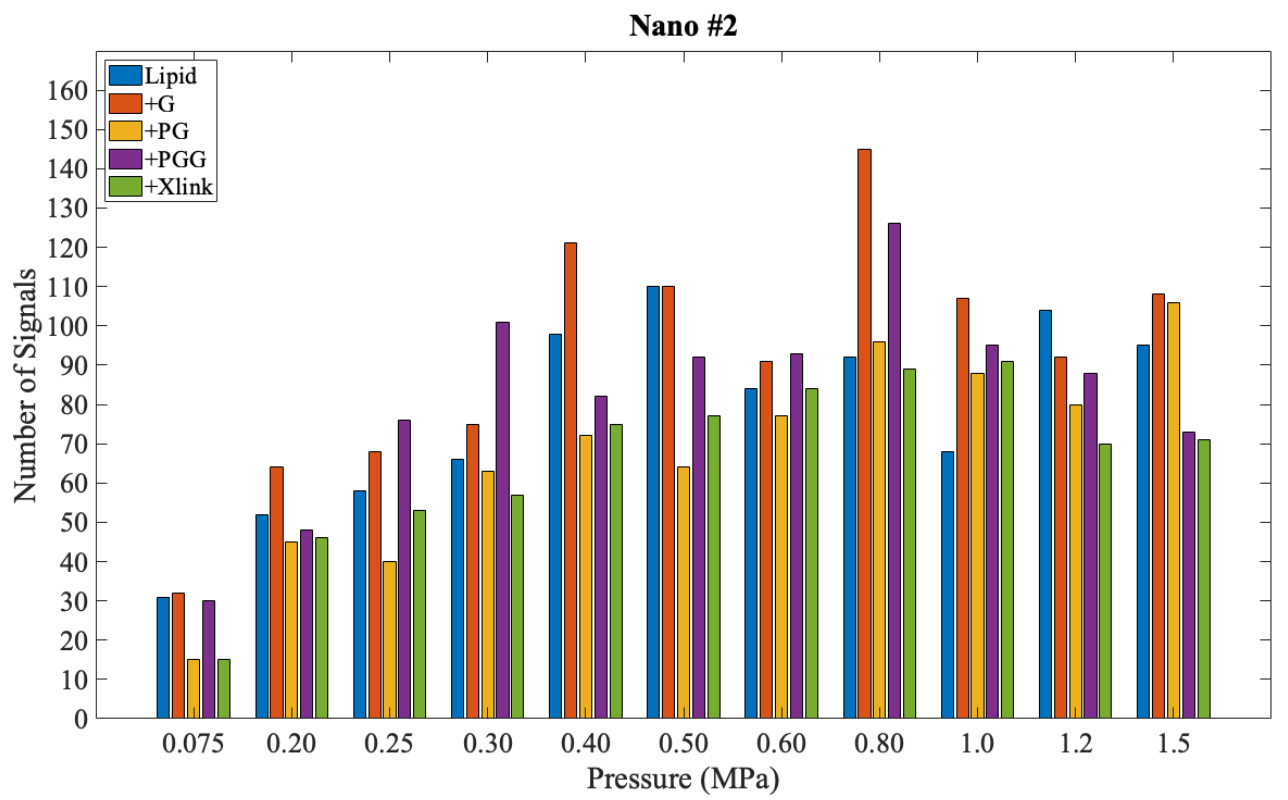

(в) 15 signals (PG and Xlink 3\% power)

FIGURE 3.2: Total number of classified bubbles across pressure for both of the nanobubble experiments. The sub-caption below each graph shows the lowest number of classified signals for each experiment. 

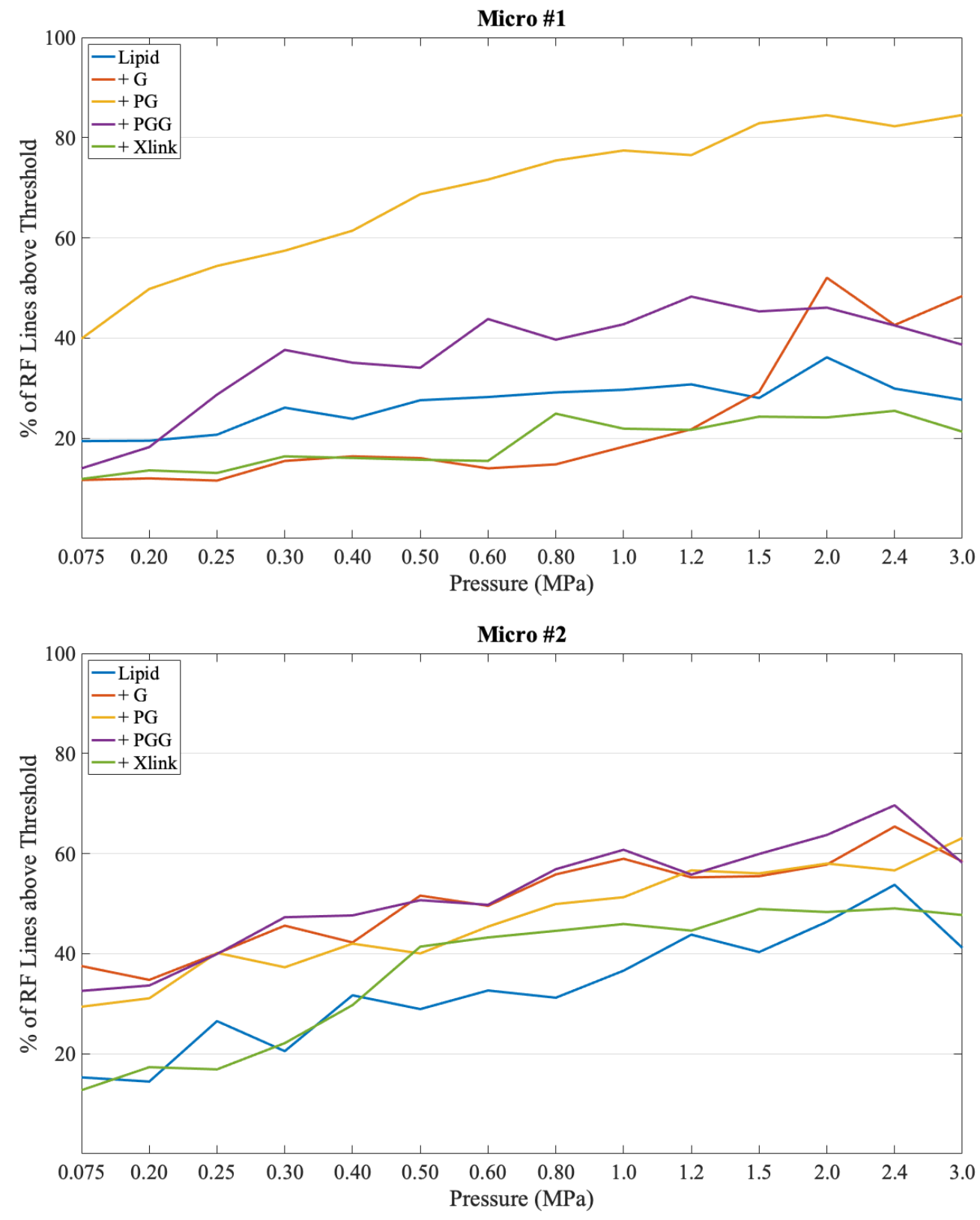

FIGURE 3.3: The percentage of RF lines for both of the microbubble experiments that pass the initial criteria of a maximum power spectrum greater than $0 \mathrm{~dB}$ in the algorithm for signal selection. 

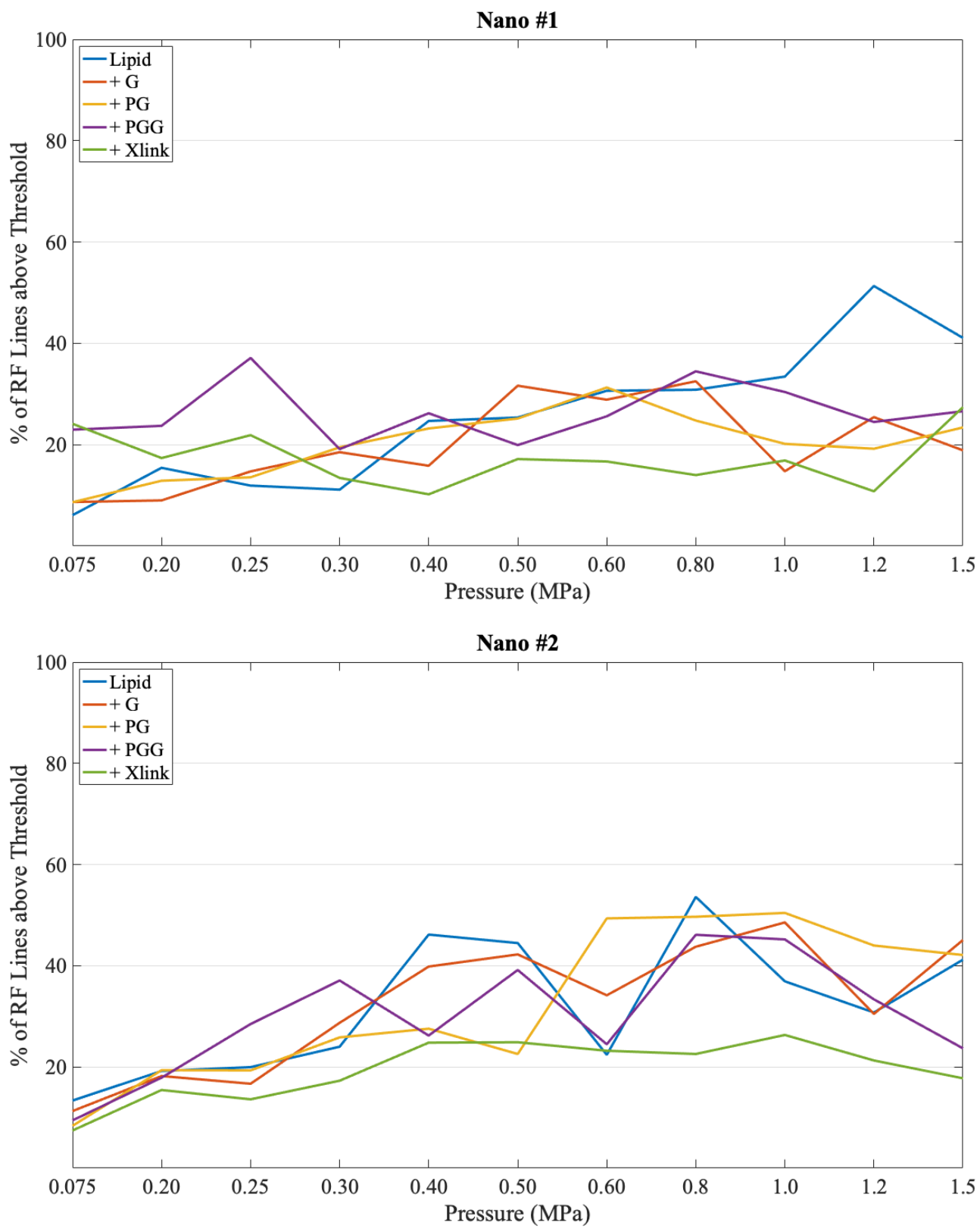

FIGURE 3.4: The percentage of RF lines for the nanobubble experiments that pass the initial criteria of a maximum power spectrum greater than $0 \mathrm{~dB}$ in the algorithm for signal selection. 


\subsubsection{Signal Counts}

Figure 3.5 shows the total count of signals of accepted, split into linear (p1) oscillations and non-linear oscillations. This data considers all pressures and shows that the counts for each experiment are not all the same. Figure 3.6 shows a breakdown of the types of non-linear signals that are included in the total in figure 3.5. Again there are considerable differences in the frequency of the detection of different bubble oscillations.

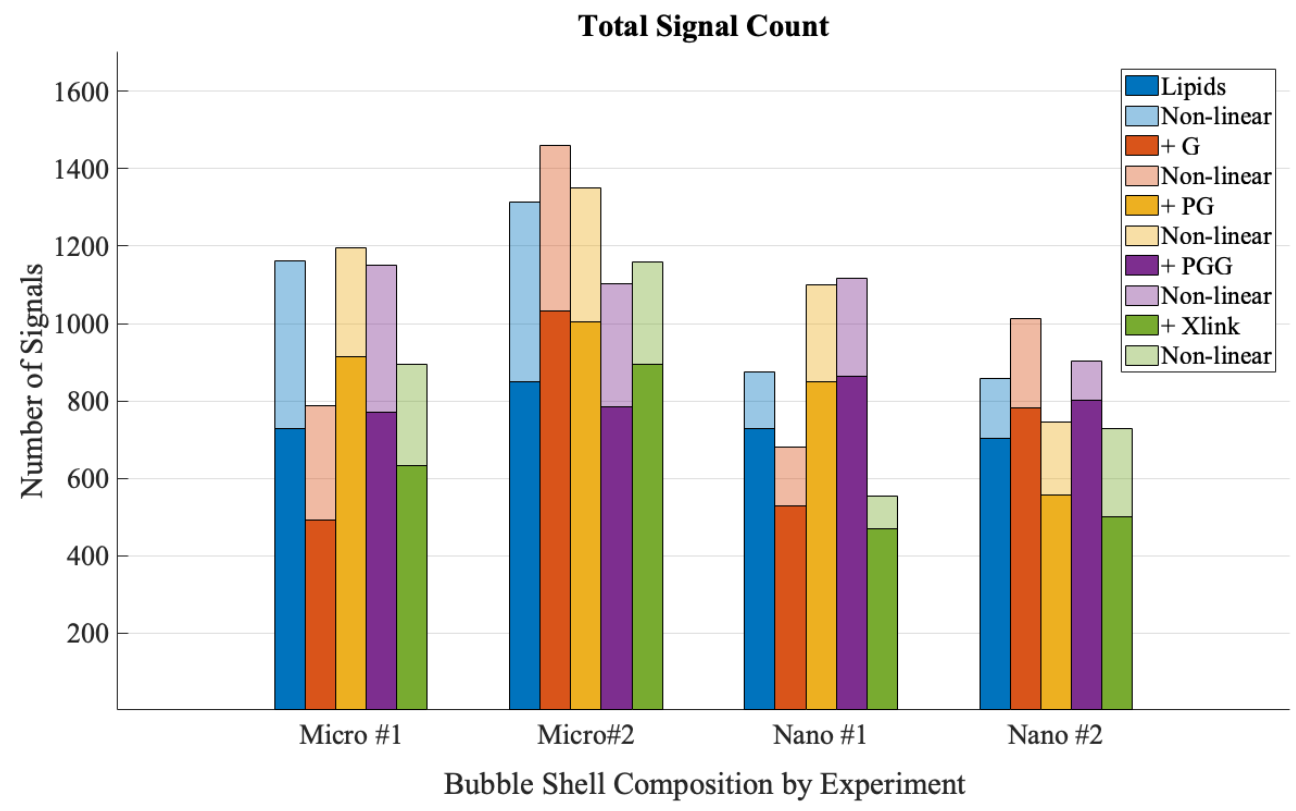

FIGURE 3.5: The total number of all signals for each experiment including all classified signals summed together, separated by the linear total (on the bottom) and the non-linear total (on the top)

\subsubsection{Percent Total Count}

The total count of signals accepted is informative but is not necessarily comparable from sample to sample as there can be external factors effecting bubble samples that could alter the bubble count from one experiment to the next. A more informative approach is to consider the percentage of signals in each category compared to all accepted signals. This method of analysis offers a better understanding of the specific non-linear behaviour in a sample of bubbles that can be compared across the different shells. This is shown 
Micro \#1

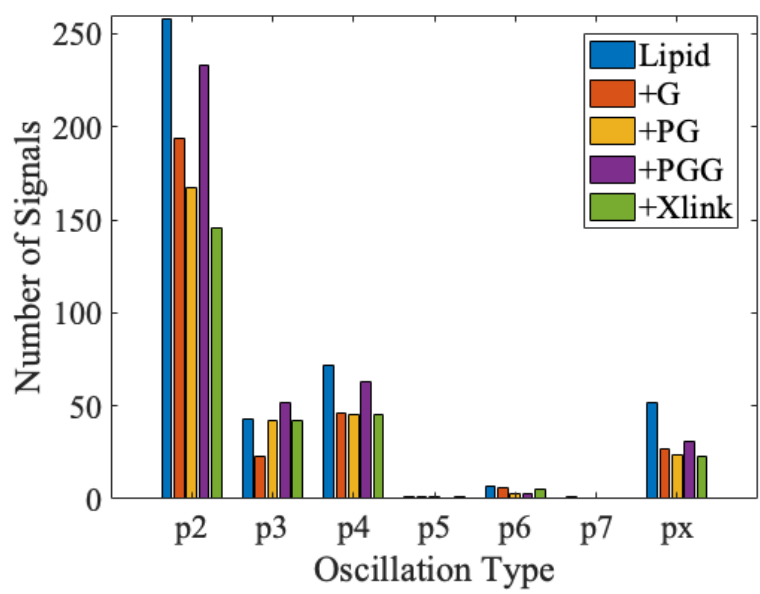

Nano \#1

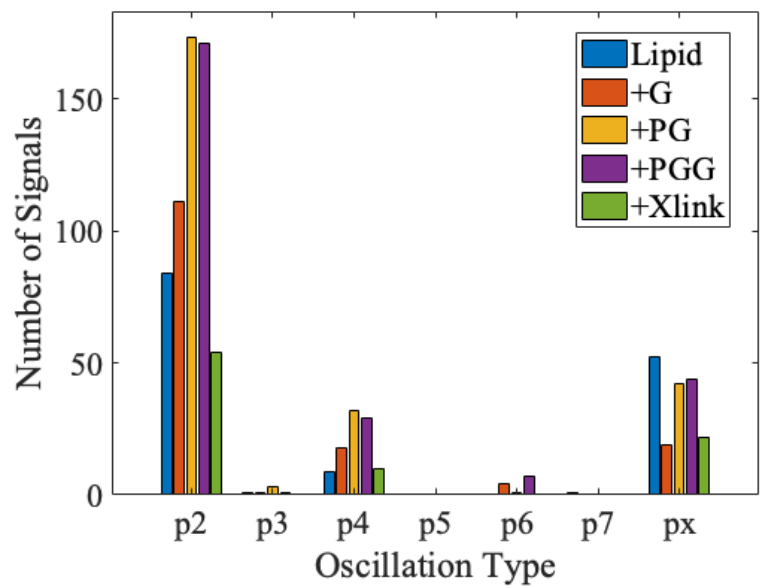

Micro \#2

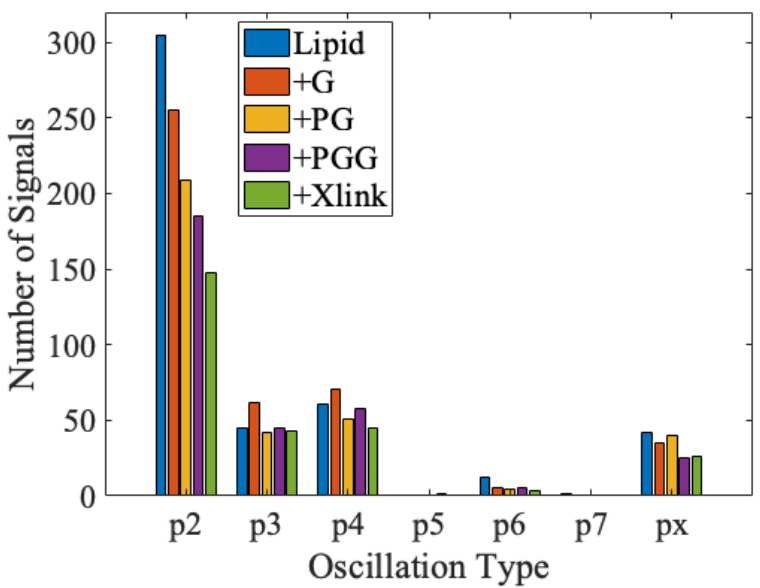

Nano \#2

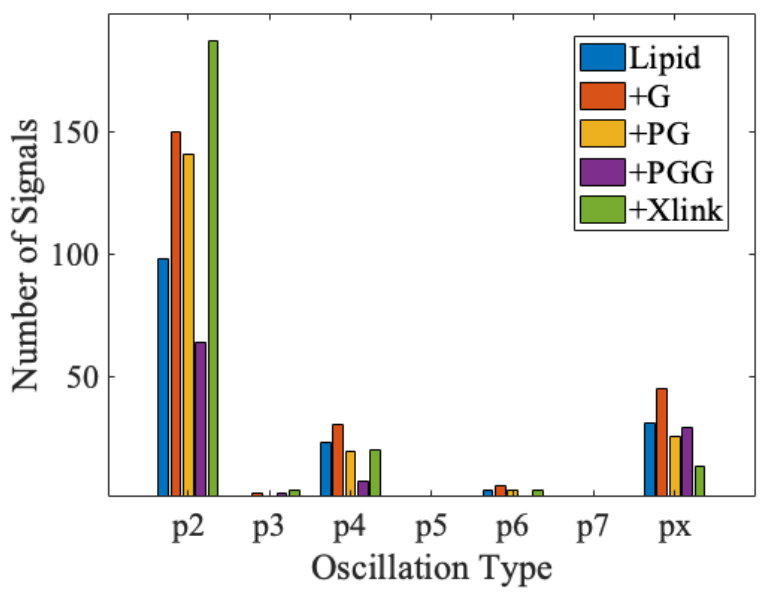

FIGURE 3.6: Total number of non-linear signals for each type of non-linear classification shown by experiment. 


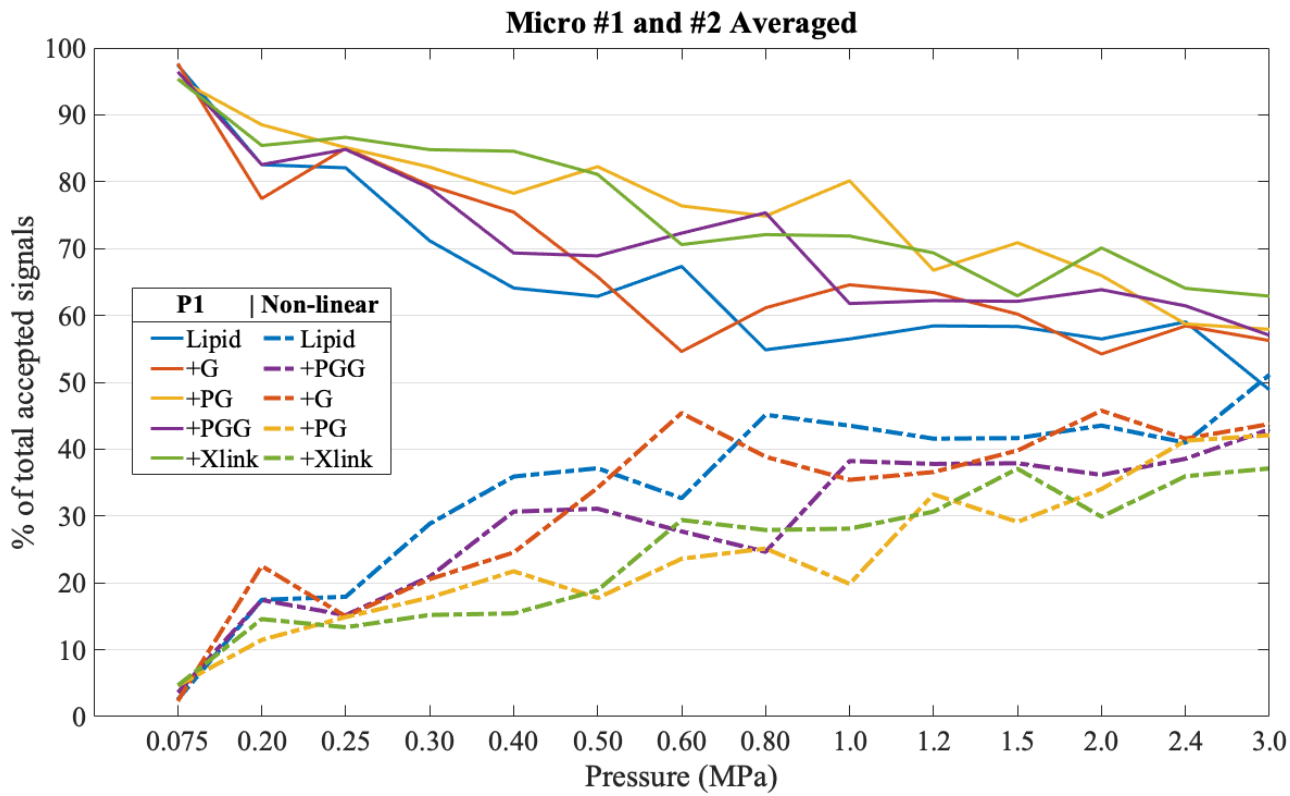

FIGURE 3.7: Average percentage of non-linear signals for microbubbles over both experiments.

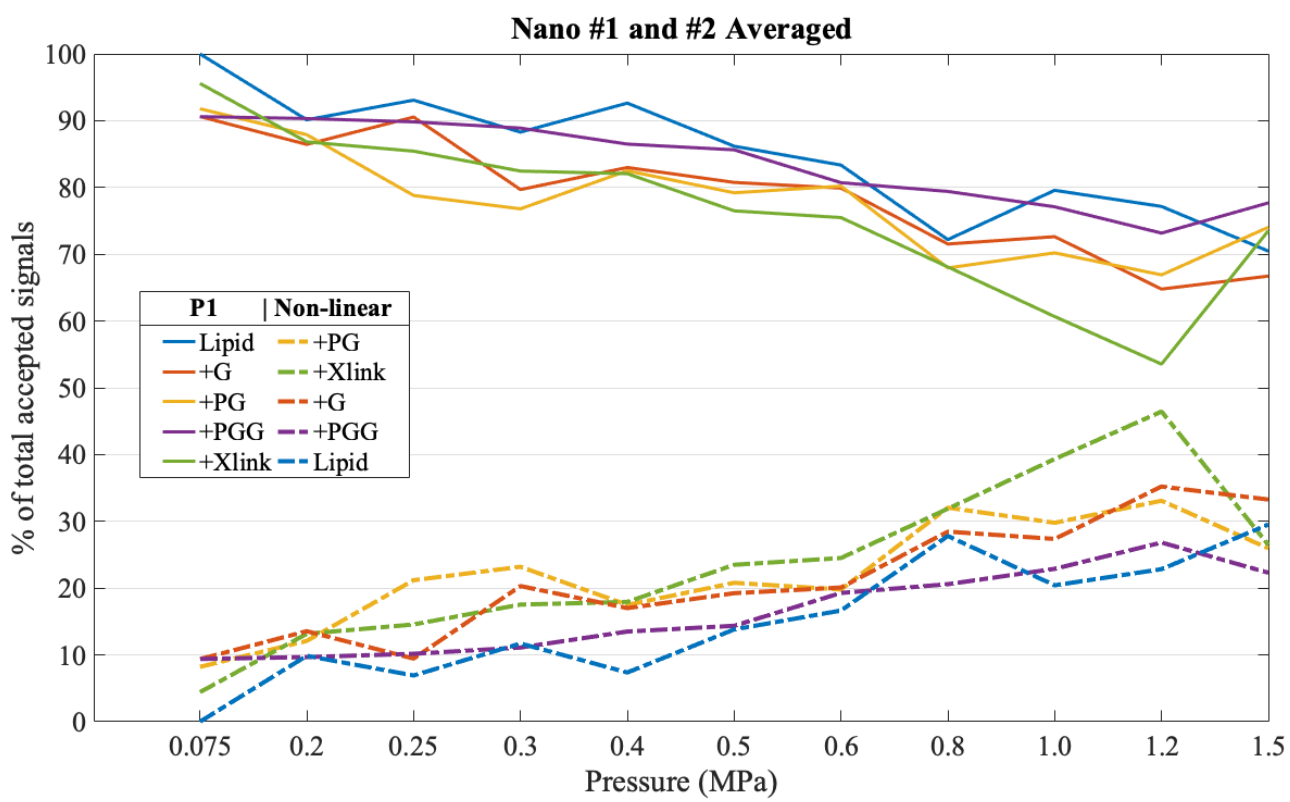

FIGURE 3.8: Average percentage of non-linear signals for nanobubbles over both experiments. 
for the microbubbles in Figure 3.7 and for the nanobubbles in Figure 3.8. These graphs show the percentage of accepted bubble signals that are undergoing p1 (linear) oscillations and non-linear oscillations for each different bubble shell type and pressure. The data in Figures 3.7 and 3.8, for microbubbles and nanobubbles respectively, averaged over both experiments. Since any signal that has been accepted that has not been labeled p1 is nonlinear, it is expected that these plots are mirror images of each other, offering a quick visual understanding of the how non-linear oscillations depend on incident pressure.

\subsection{Amplitude Analysis}

\subsubsection{Fundamental Amplitude Analysis}

Next, the fundamental amplitude of the linear and non-linear signals were analyzed by finding the max peak of the power spectrum in $\mathrm{dB}$ and averaging across all $\mathrm{p} 1$ and all non-linear signals separately, for each pressure, for each bubble shell type. These graphs can be seen in arranged by size and experiment in Figure 3.9 and 3.10 for the linear signals and in Figure 3.11 and 3.12 for the non-linear signals. The error bars represent the standard deviation for all signals collected (whether $\mathrm{p} 1$ or all non-linear signals) to determine the mean for the maximum amplitude.

\subsubsection{Subharmonic and Ultra Harmonic Amplitude Analysis}

After considering the maximum amplitude of the fundamental, the amplitudes of the sub harmonic and ultra harmonic frequencies were evaluated. This was done in a similar way to the fundamental taking the max $\mathrm{dB}$ in the power spectrum for the specified peak. Figure 3.13 offers a visual explanation as to how these peaks were identified and measured. Since we know where the subharmonic and ultra harmonic peaks can be found in position relative to the fundamental their maxima were located by searching for peaks in a frequency 

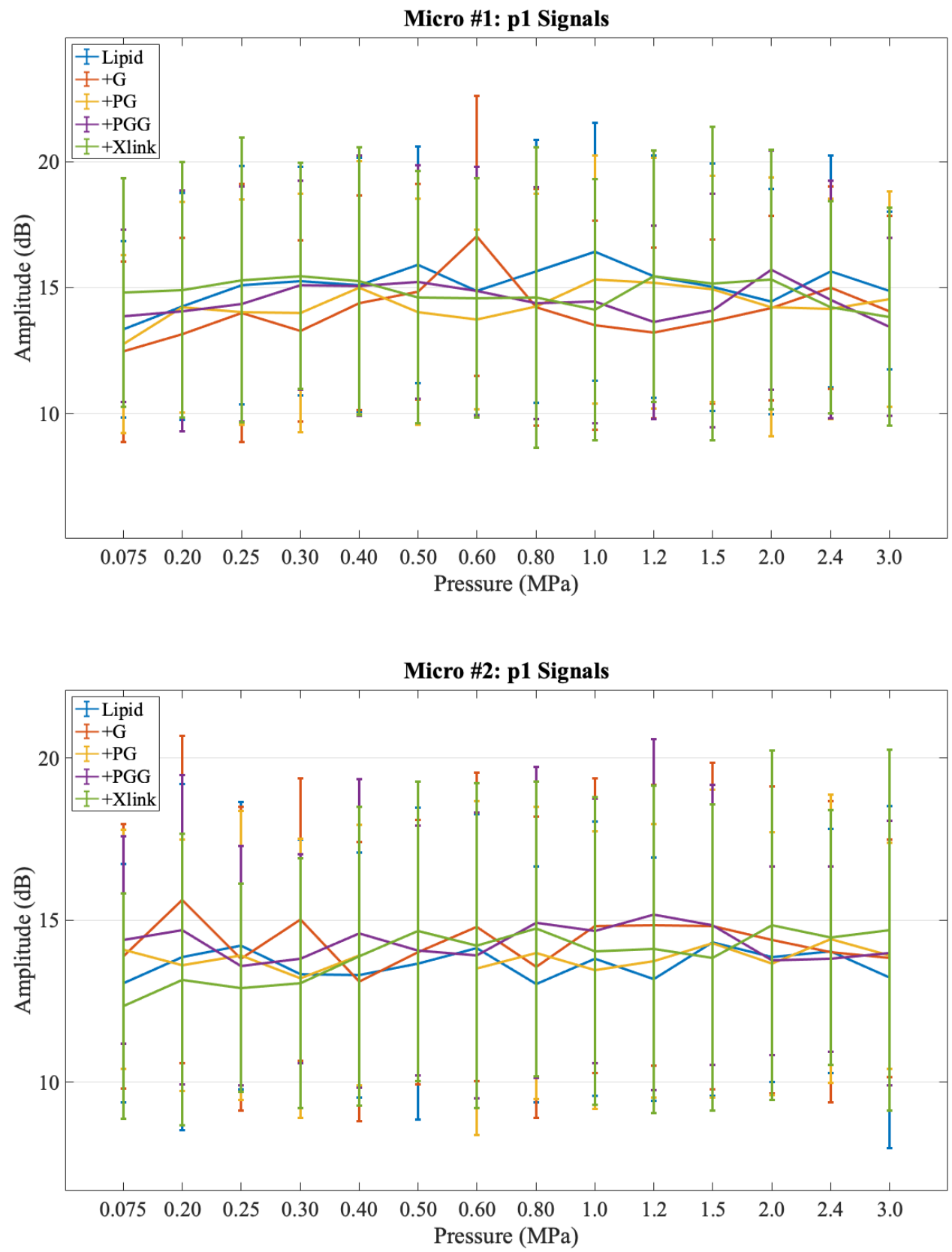

FIGURE 3.9: Mean fundamental amplitude of p1 signals for microbubbles \#1 (top) and \#2 (bottom) 

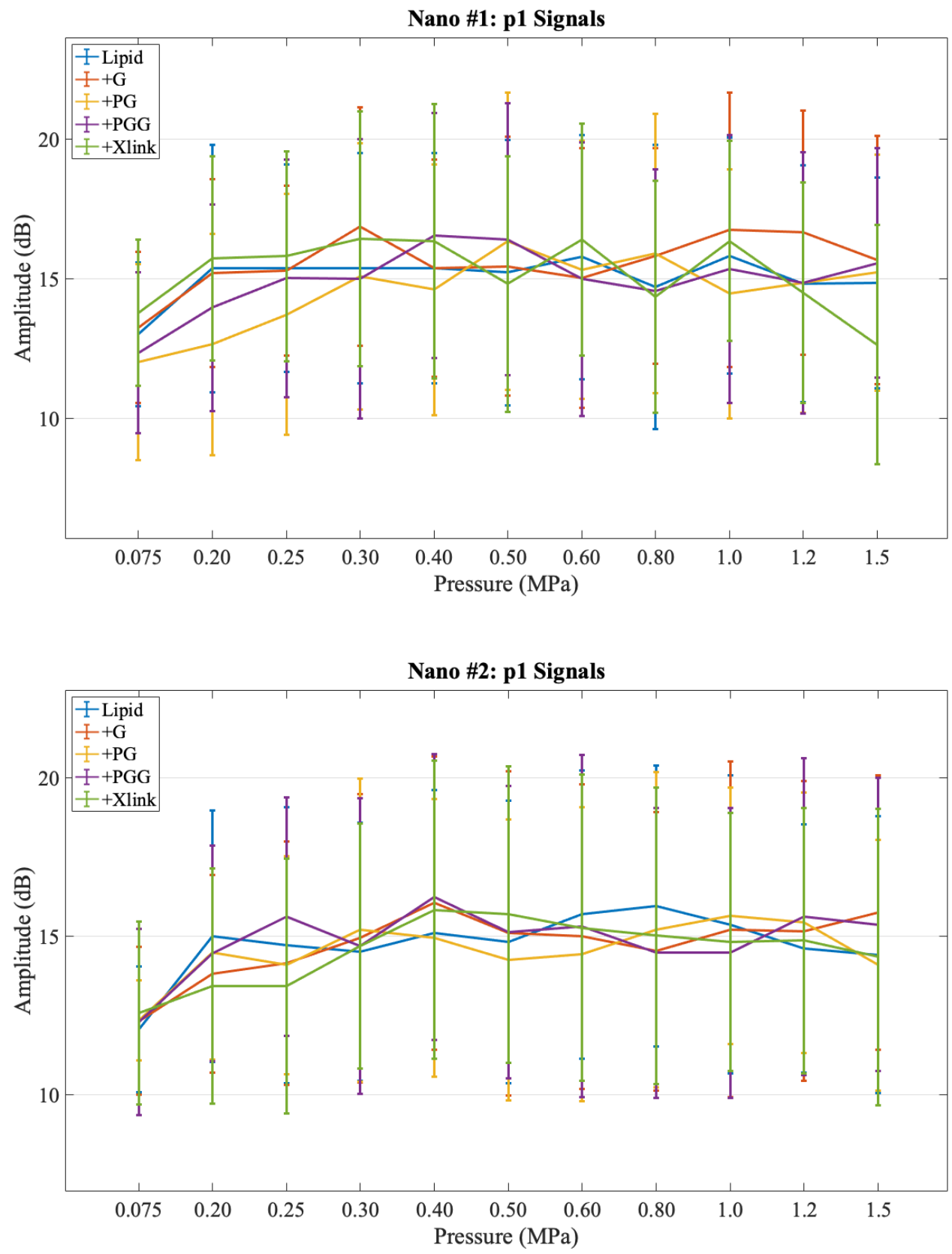

FIGURE 3.10: Mean fundamental amplitude of $\mathrm{p} 1$ signals for nanobubbles \#1 (top) and \#2 (bottom) 

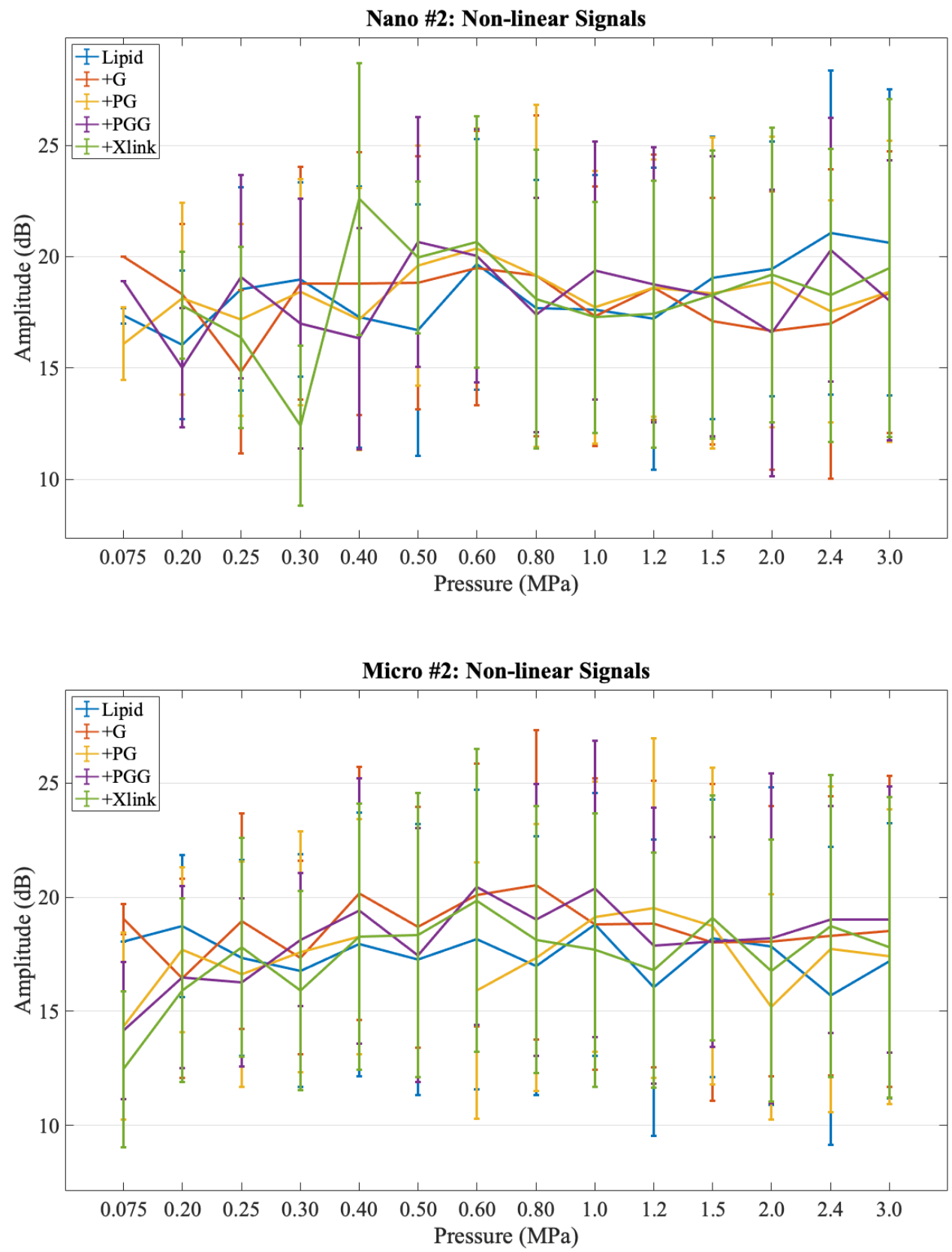

FIGURE 3.11: Mean fundamental amplitude of non-linear signals for microbubble experiments \#1 (top) and \#2 (bottom) 

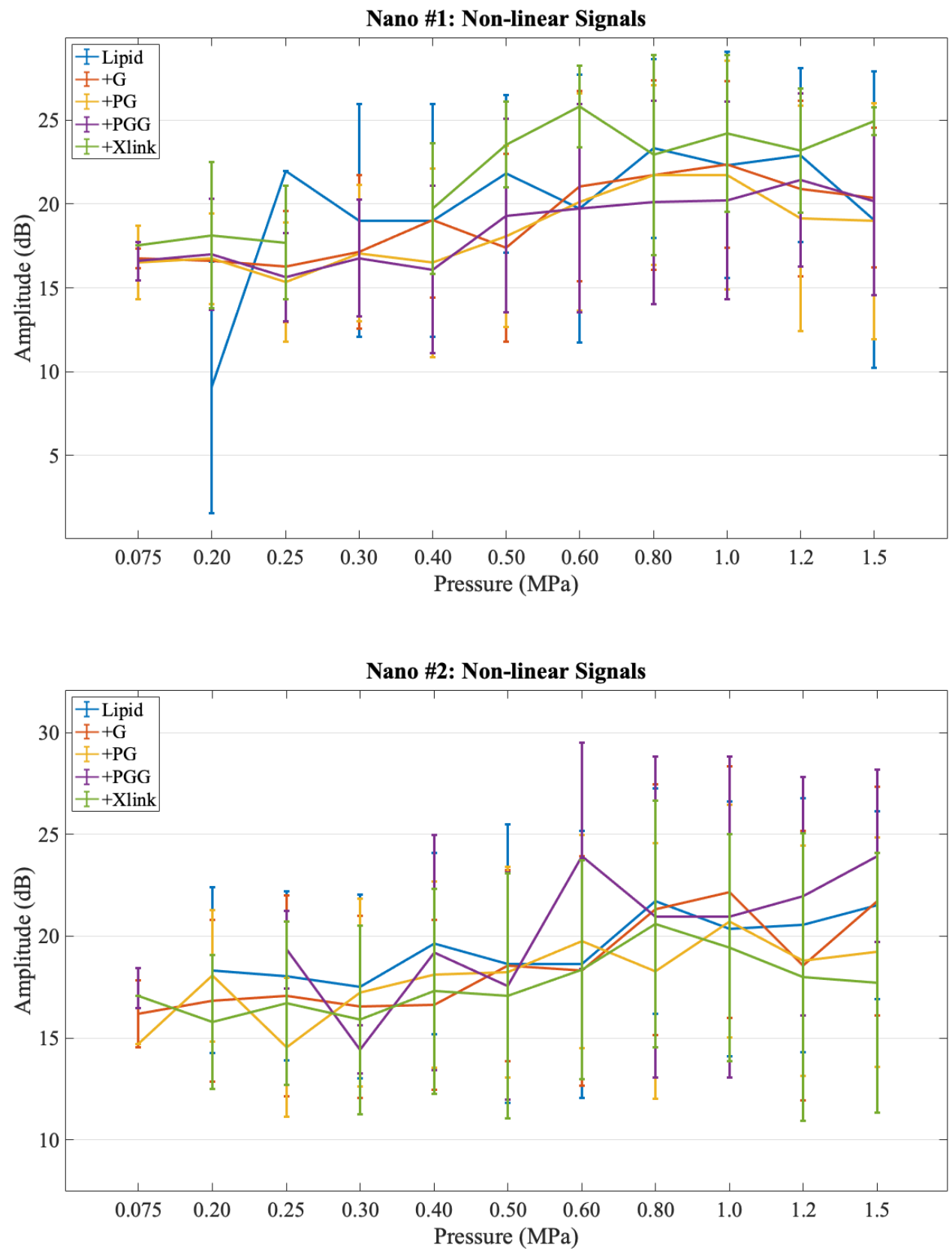

FIGURE 3.12: Mean fundamental amplitude of non-linear signals for nanobubble experiments \#1 (top) and \#2 (bottom) 


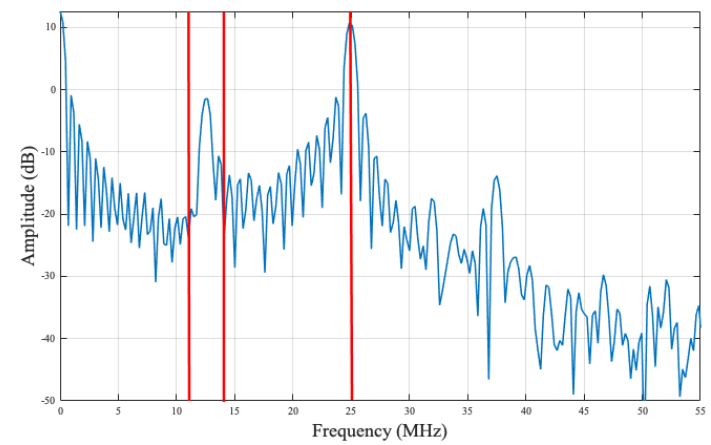

FIGURE 3.13: A demonstration of how subharmonic and ultra harmonic peaks in the power spectrum are located by using fractions of the fundamental peak position.

range relative to the fundmentals' position. For example, the subharmonic of both p2 and the strongest subharmonic of $\mathrm{p} 4$ is located at $\frac{1}{2}$ of the frequency of the fundamental.

The peaks that were considered for the analysis of the sub-and ultra harmonics are highlighted by red circles in Figure 3.14 (for p2, p3, and p4 signals).

The graphs in Figures 3.15 and 3.16 show the fundamental, subharmonic, and ultra harmonic maximum amplitudes all represented in one graph each for the p2 and p4 nonlinear scattering cases. The same data can be seen for the Nano \#1 experiments in Figures 3.17 and 3.18 .

To determine their relative strength the subharmonic and ultra harmonic average amplitudes were subtracted from that of the fundamental. The y-axis represents the difference between the fundamental and subharmonic amplitudes (shown in Figure 3.19) and between the fundamental and ultra harmonic amplitudes (shown in Figure 3.20) for the Micro \#1 experiment. The Nano \#1 experiment is shown in Figure 3.21 for the fundamental and subharmonic difference and in Figure 3.22 for the fundamental and ultra harmonic difference. 

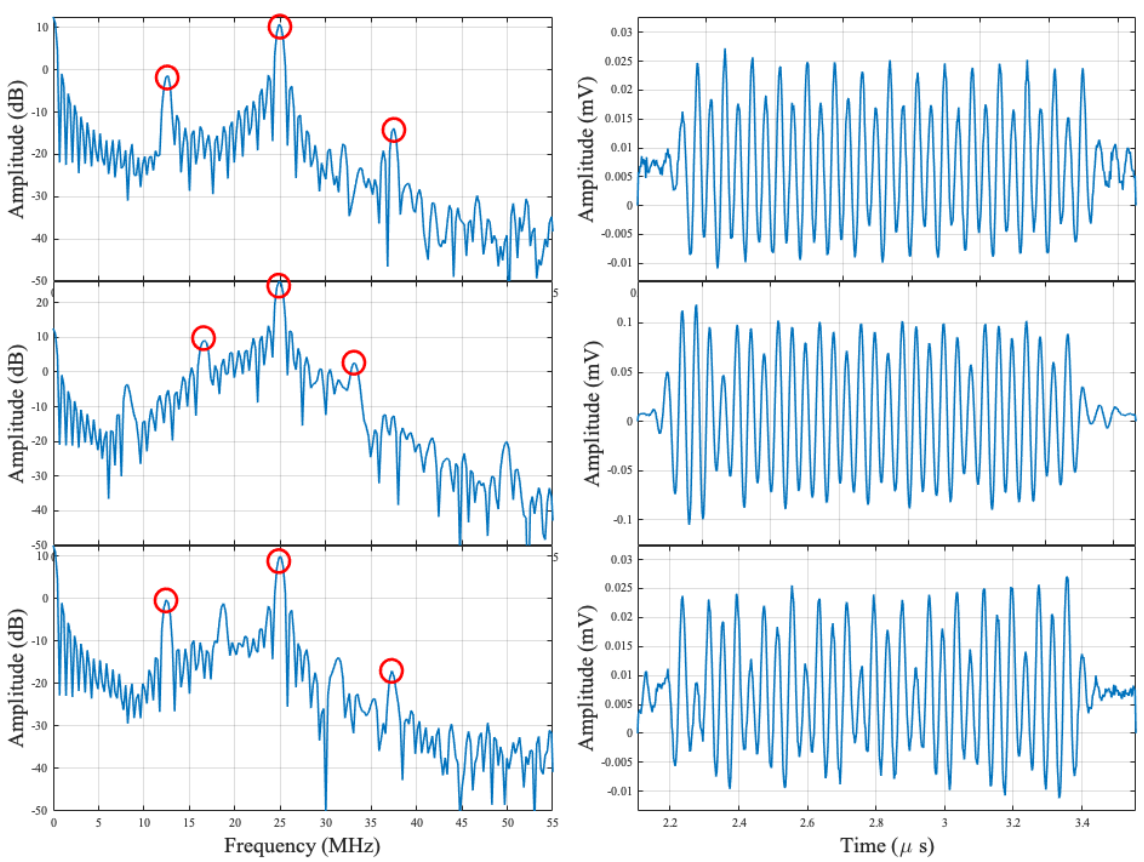

FIGURE 3.14: Sample power spectra and RF data for p2 (top), p3 (middle) and p4 (bottom) demonstrating the harmonic peaks that were analyzed with a circle highlighting them.

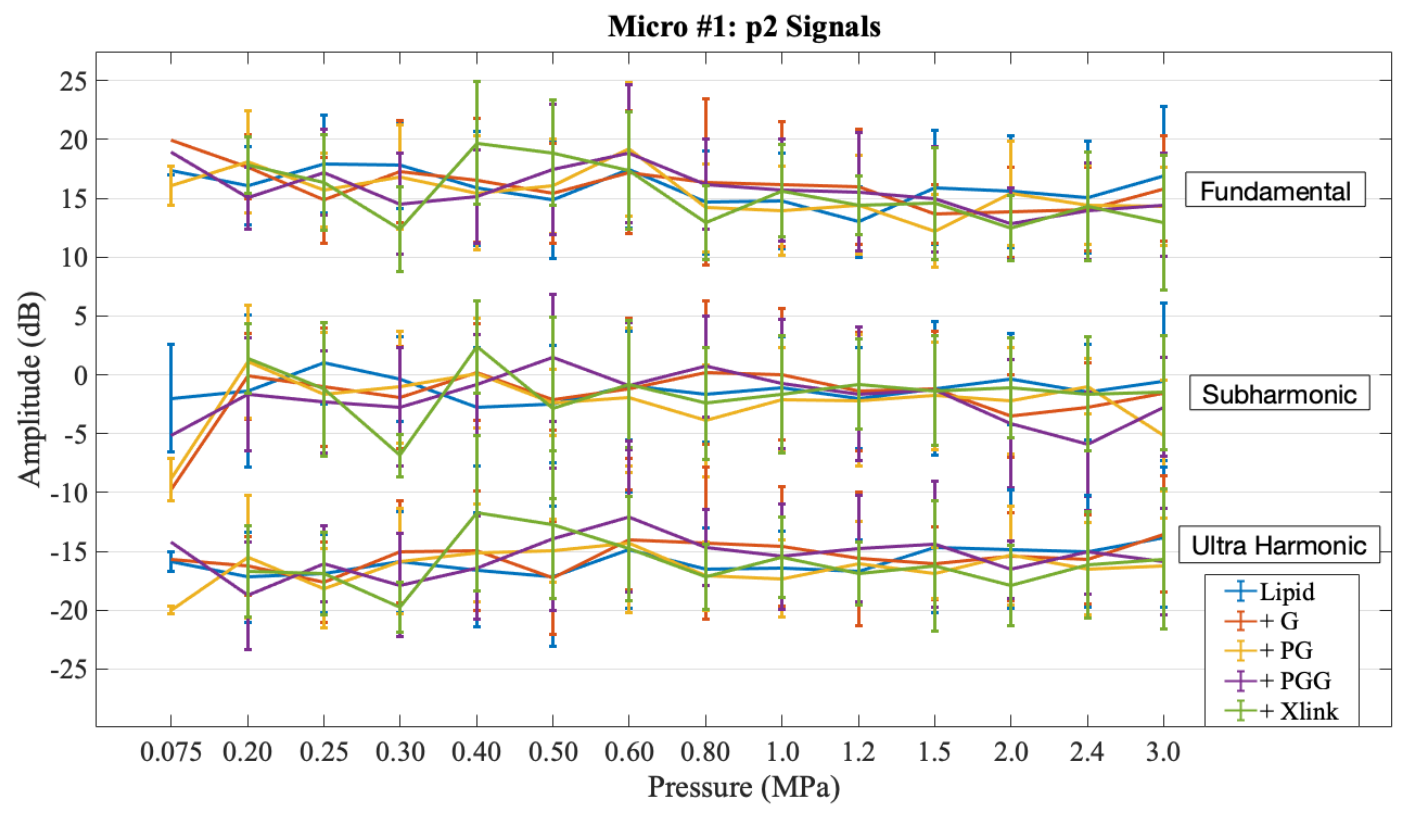

FIGURE 3.15: Micro \#1: fundamental, subharmonic and ultra harmonic average amplitudes for $\mathrm{p} 2$ signals as a function of pressure for each shell type 


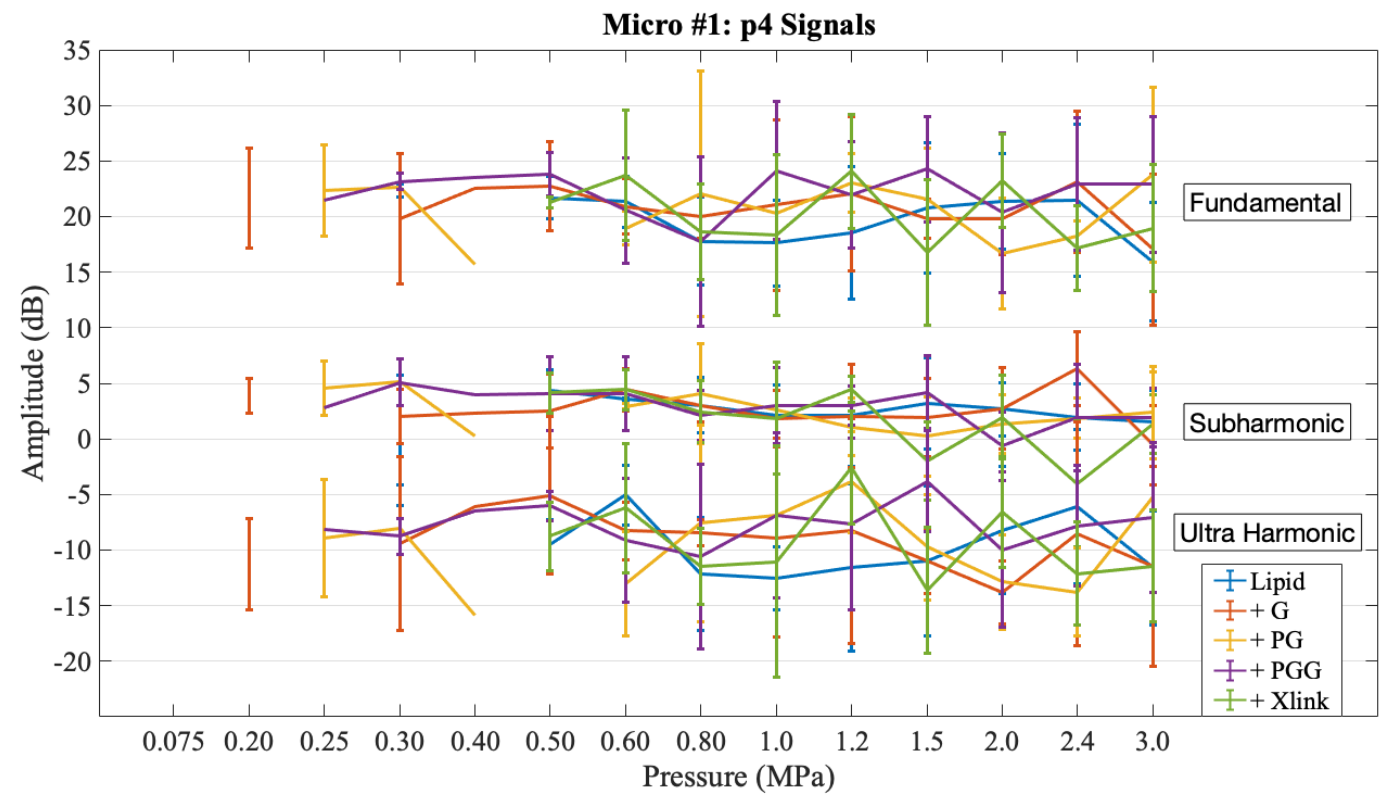

FIGURE 3.16: Micro \#1: fundamental, subharmonic and ultra harmonic average amplitudes for $\mathrm{p} 4$ signals as a function of pressure for each shell type

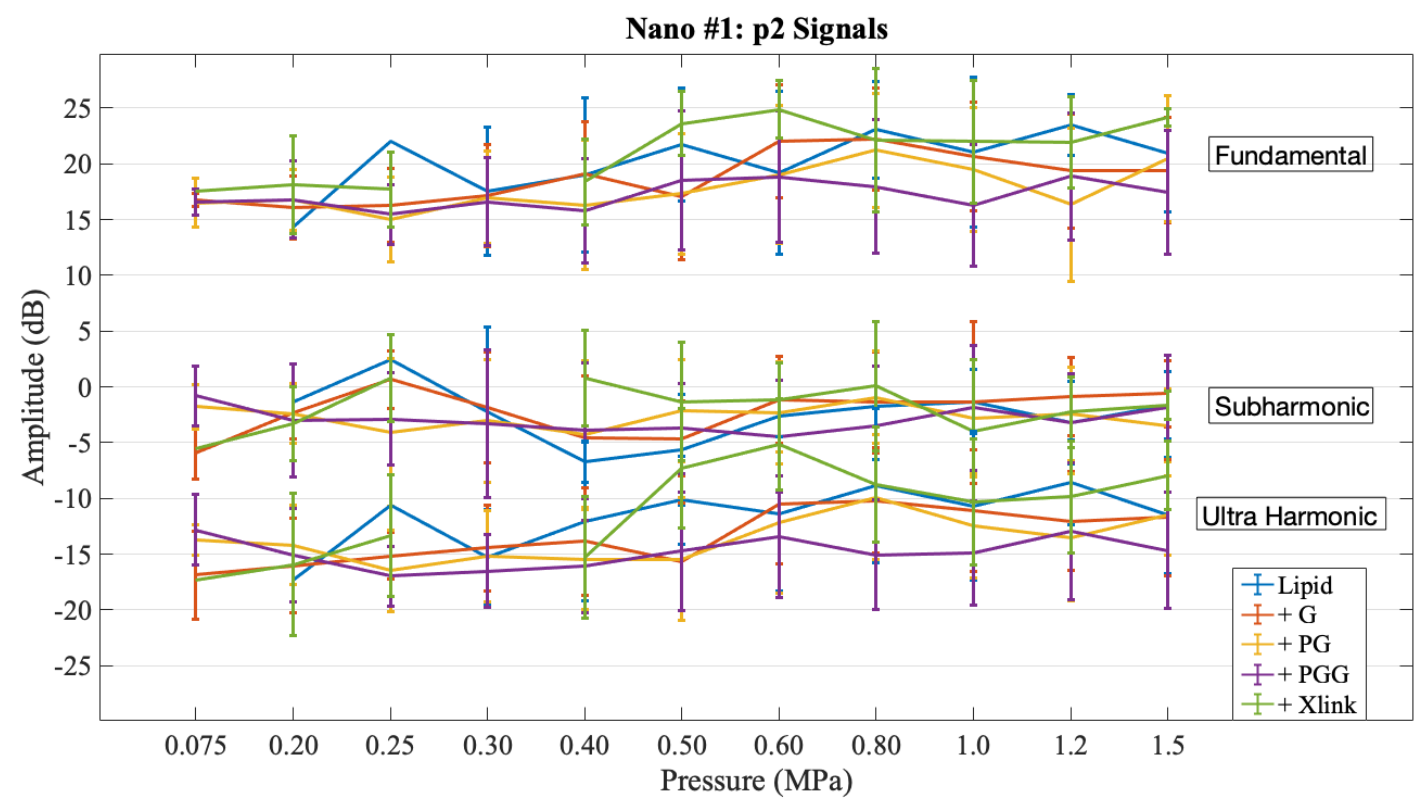

FIGURE 3.17: Nano \#1: Fundamental, Subharmonic and Ultra Harmonic average amplitudes for $\mathrm{p} 2$ signals as a function of pressure for each shell type. 


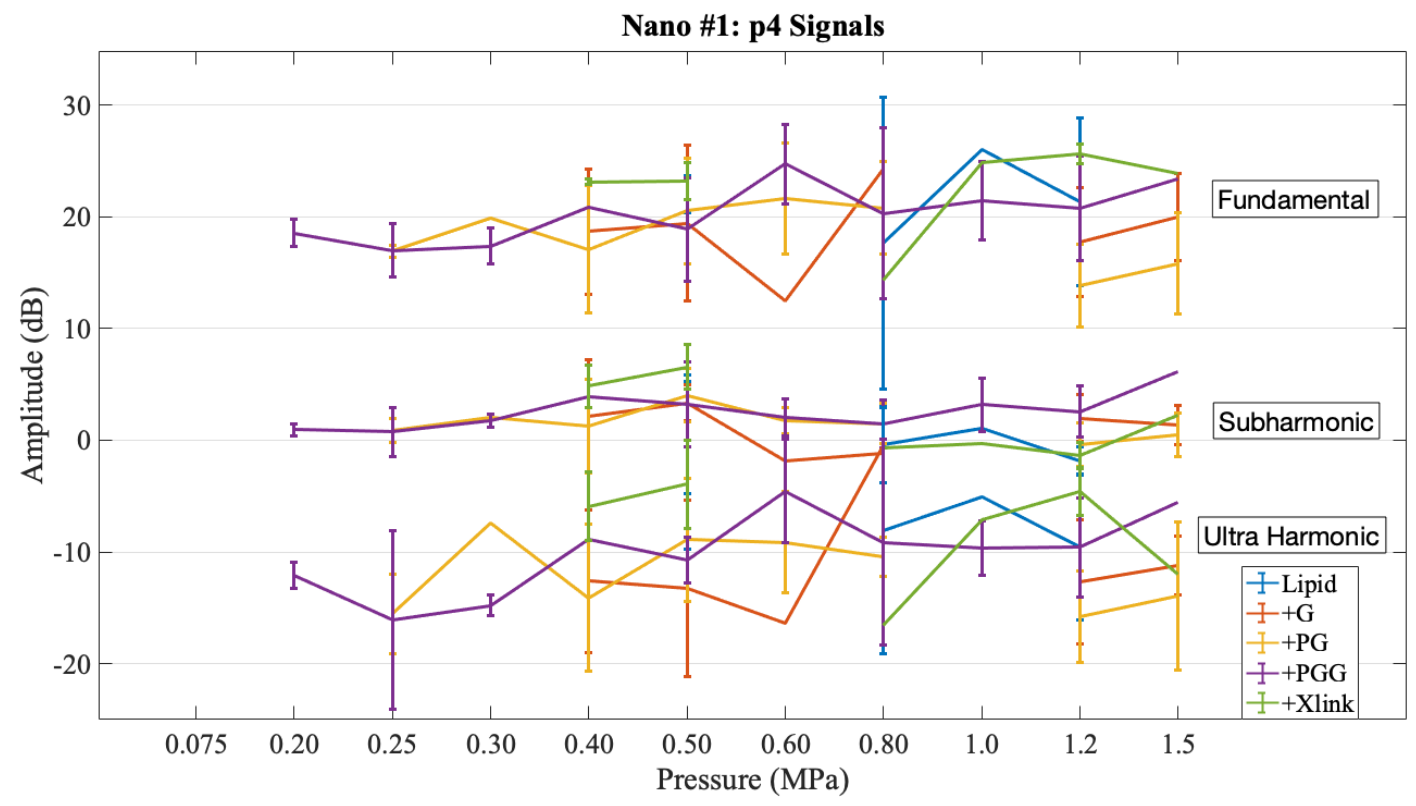

FIGURE 3.18: Nano \#1: fundamental, subharmonic and ultra harmonic average amplitudes for $\mathrm{p} 4$ signals as a function of pressure for each shell type. 

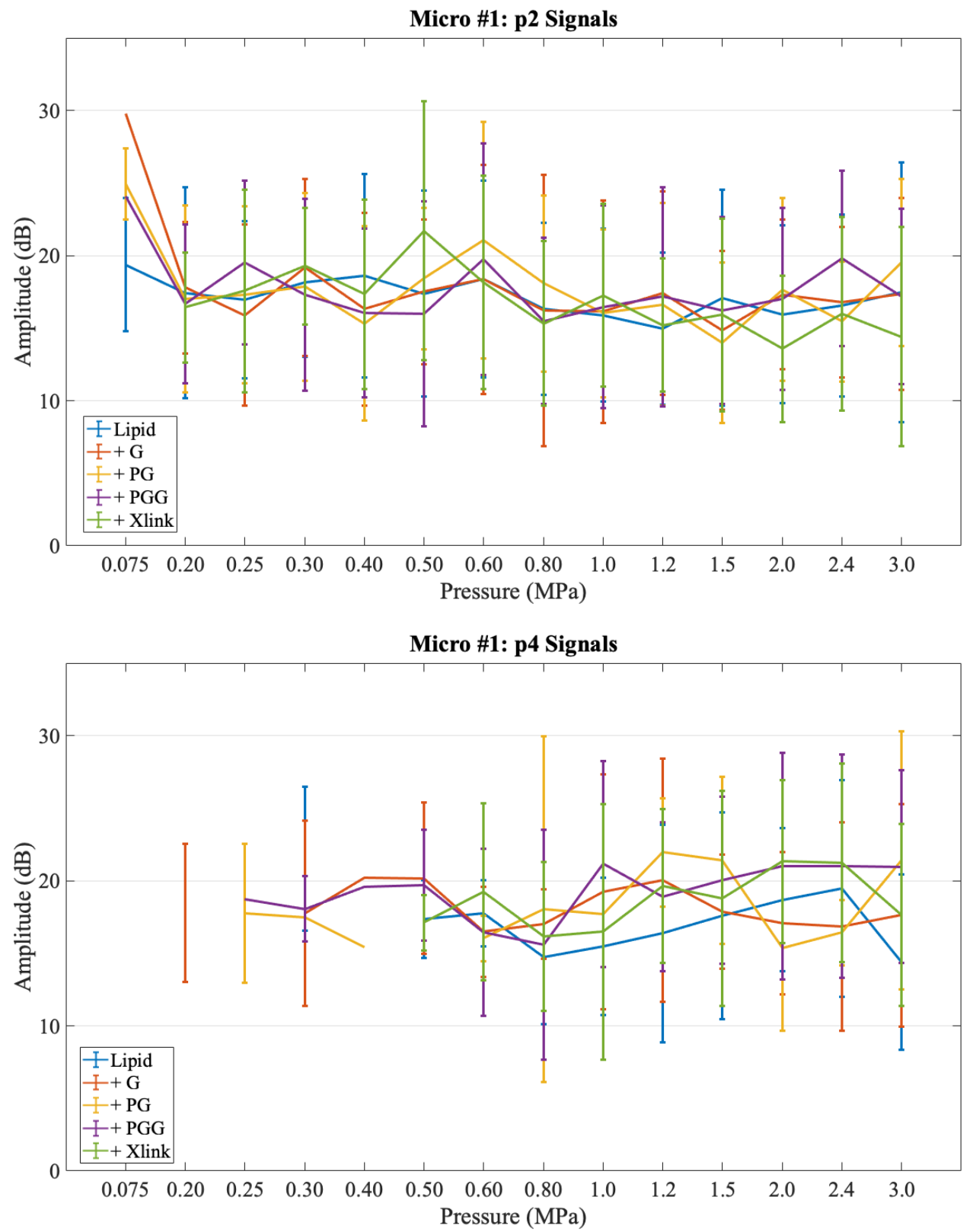

FIGURE 3.19: Difference between fundamental to subharmonic as a function of pressure for microbubbles for p2 (top) and p4 (bottom) signals 

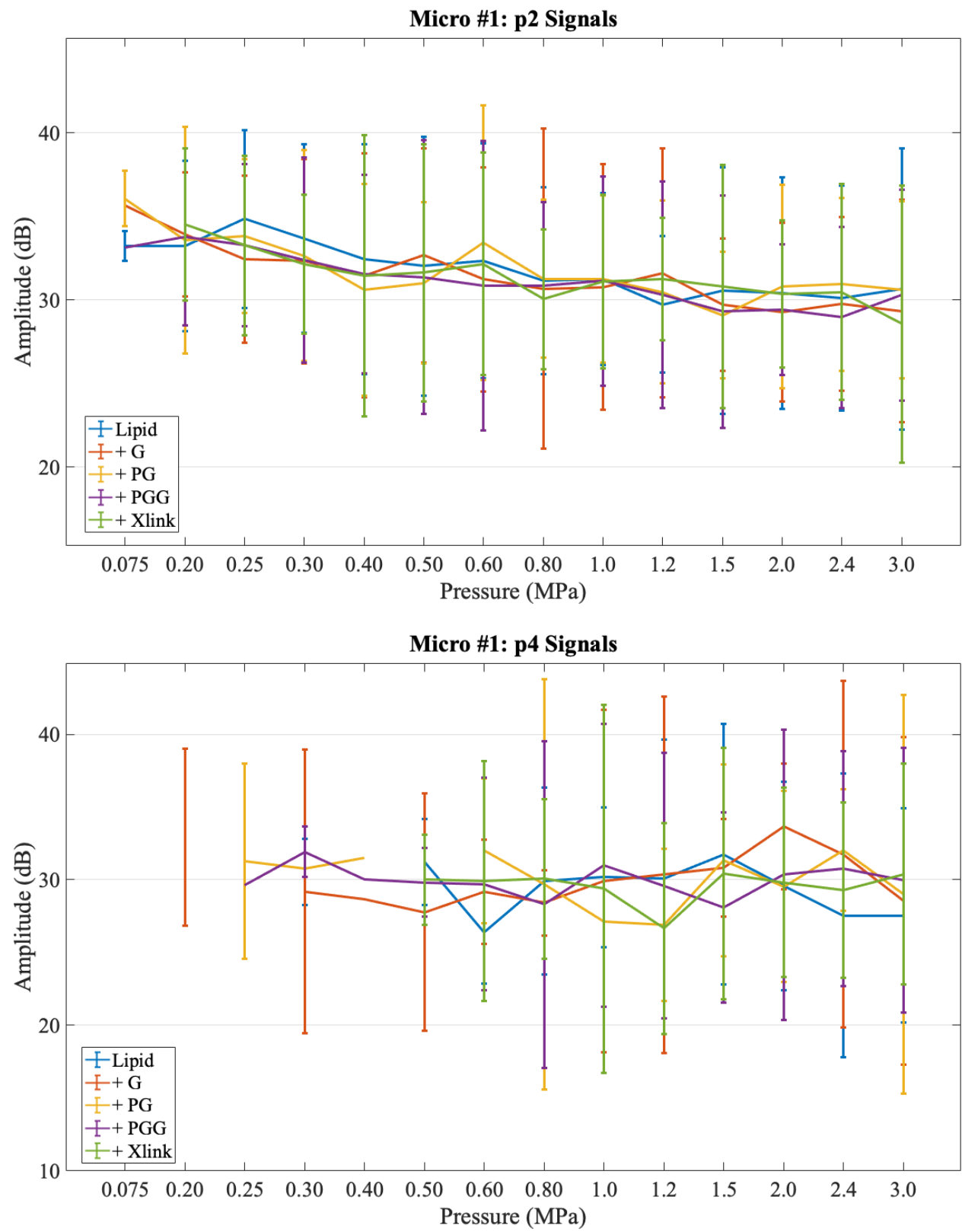

FIGURE 3.20: Difference between fundamental to ultra harmonic as a function of pressure for microbubbles for p2 (top) and p4 (bottom) signals 

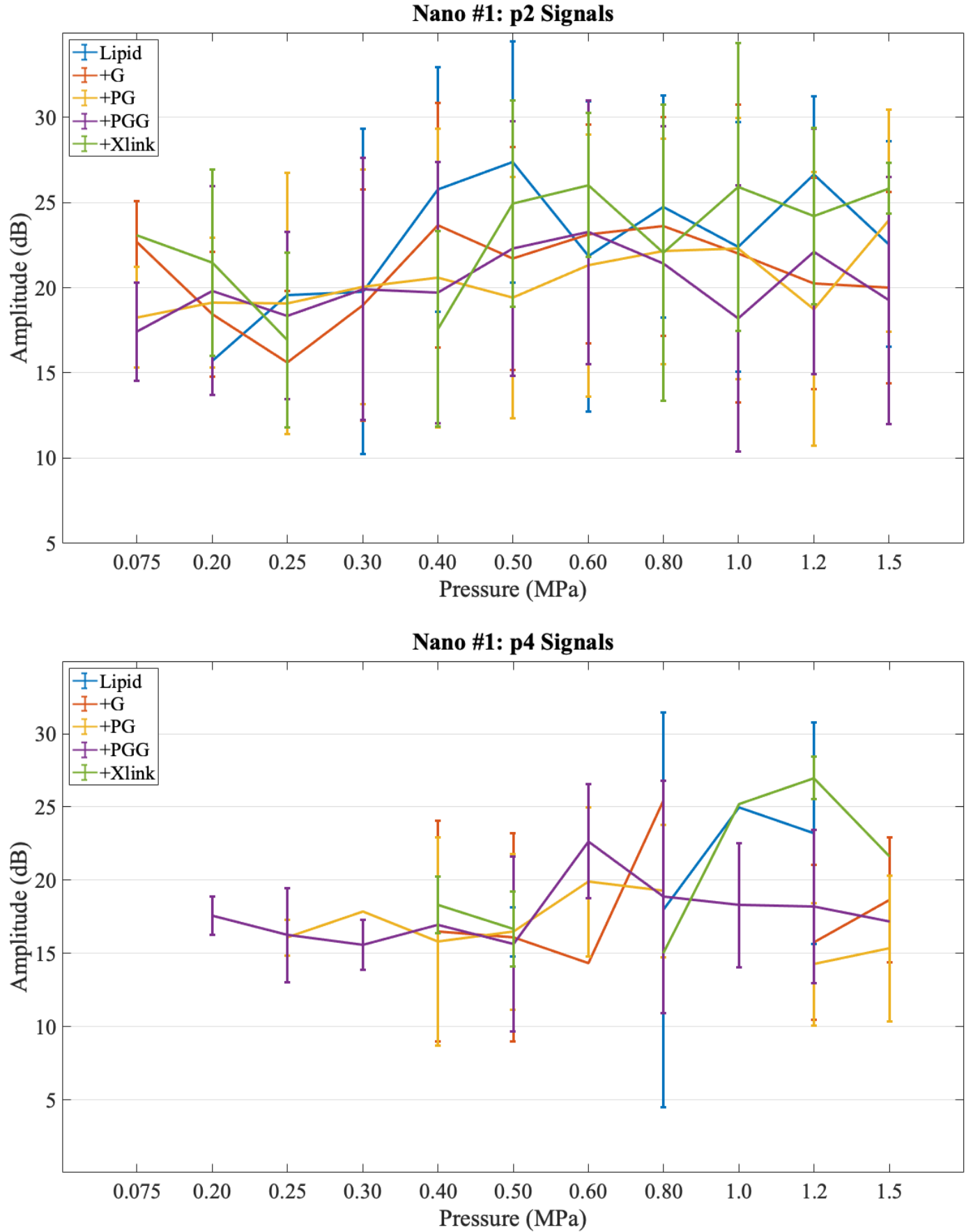

FIGURE 3.21: Difference between fundamental and subharmonic as a function of pressure for nanobubbles for $\mathrm{p} 2$ (top) and $\mathrm{p} 4$ (bottom) signals. 
Nano \#1: p2 Signals
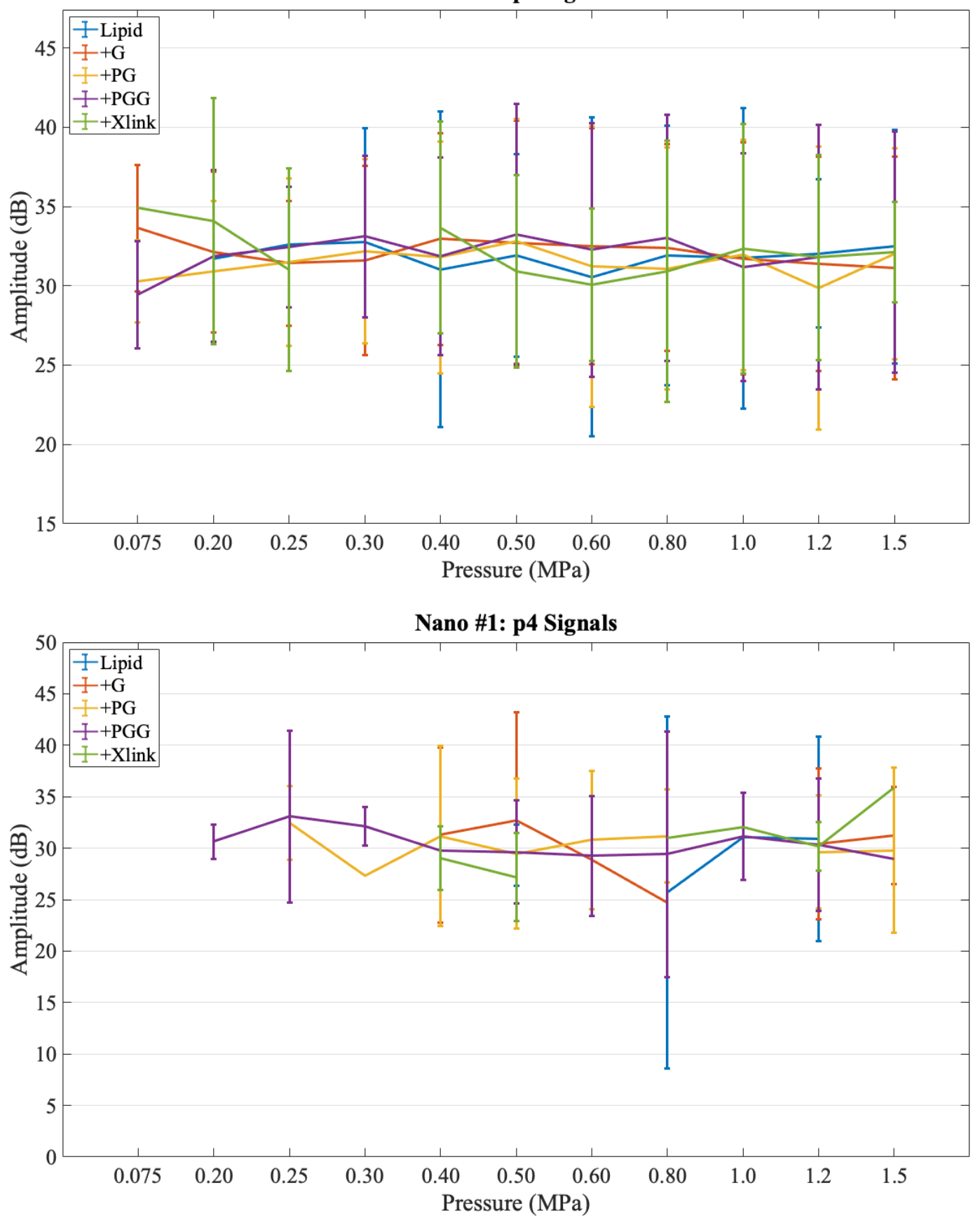

FIGURE 3.22: Difference between fundamental and ultra harmonic as a function of pressure for nanobubbles for $\mathrm{p} 2$ (top) and $\mathrm{p} 4$ (bottom) signals. 


\section{Chapter 4}

\section{Discussion}

\subsection{The Effect of Bubble Shell Composition on UCA Behaviour}

The signal statistics show variation in section 3.2.2 across the different shell compositions. The results found are reproducible nevertheless, we did not see a trend in comparing shell composition across pressure and oscillation type. However, there does appear to be a trend in the differences between microbubbles and nanobubbles across pressure and oscillation type.

\subsubsection{Fundamental and Harmonic Amplitude Analysis}

The fundamental, subharmonic and ultra harmonic amplitudes were examined for p2, p3 an p4 signals as they are the most abundantly occurring non-linear signals. Through this analysis, no difference in the fundamental and harmonic amplitudes were measured for the bubbles of different shell composition. The difference between the fundamental and the subharmonic and the fundamental and the ultra harmonic was averaged across p2, p3, and $\mathrm{p} 4$ to determine if there were any difference evident across different non-linear signals. There was no trend shown through this method of analysis. The graphs that show this are in Appendix B, Figures B.3 and B.4. 


\subsubsection{Size Distribution Dependence}

One reason there may be detectable differences in non-linear oscillation patterns for the different shell compositions is the bubble size distribution. The graphs showing the size distribution for each shell type for each size and experiment are shown in Appendix A. The size distribution may not be narrow enough to produce an observed difference in shell behaviour. The width of the size distribution translates to approximately a $4-5 \mathrm{MHz}$ shift in the resonance frequency of the UCAs used. Although the additional shell components alter the bubbles' elasticity and shell wall viscosity, this is likely not enough of a change to overcome the effects of the size distribution, as the non-linear behaviour of bubbles is highly dependent on size. For each RF line collected the exact size of the microbubble or nanobubble that gave rise to it is unknown.

The shell composition may also effect the bubble sizes and provide challenges for creating bubbles of equal size. For example, as stated in section 1.4.1, the addition of propylene glycol alters the distance between phospholipids within the shell. The shell compositions may not be created equally and perhaps have different size distribution or concentrations.

Additionally, the buckling of the shell as discussed in section 1.3.4 may dominate bubble behaviour. During the compression phase of a bubbles' oscillation the shell may buckle, causing the surface tension of the shell to drop to zero. However, the difference in shell composition may change when and if buckling occurs.

\subsection{A Comparison of Microbubbles and Nanobubbles}

\subsubsection{Initial Criteria Threshold}

The data in Figure 3.3 and Figure 3.4 shows the percentage of signals that pass the initial criteria in the algorithm for signal selection. Figure 4.1 has the comparison of 
the average of the two independent experiments for the microbubbles and nanobubbles. It can be seen that a larger percentage of the microbubbles pass the initial criteria than nanobubbles. There is approximately a $10 \%$ difference in signals that pass this first criteria between microbubbles and nanobubbles. As each $1 \%$ represents $25 \mathrm{RF}$ lines the microbubble samples on average contain 250 more signals per pressure than nanobubbles. This trend is confirmed further in the accepted signals depicted in Figure 3.5. The overall signal count as well as the non-linear signal count is higher for microbubbles. Additionally, the burst count is greater for nanobubbles, with no signal data for pressures greater than $1500 \mathrm{kPa}$.

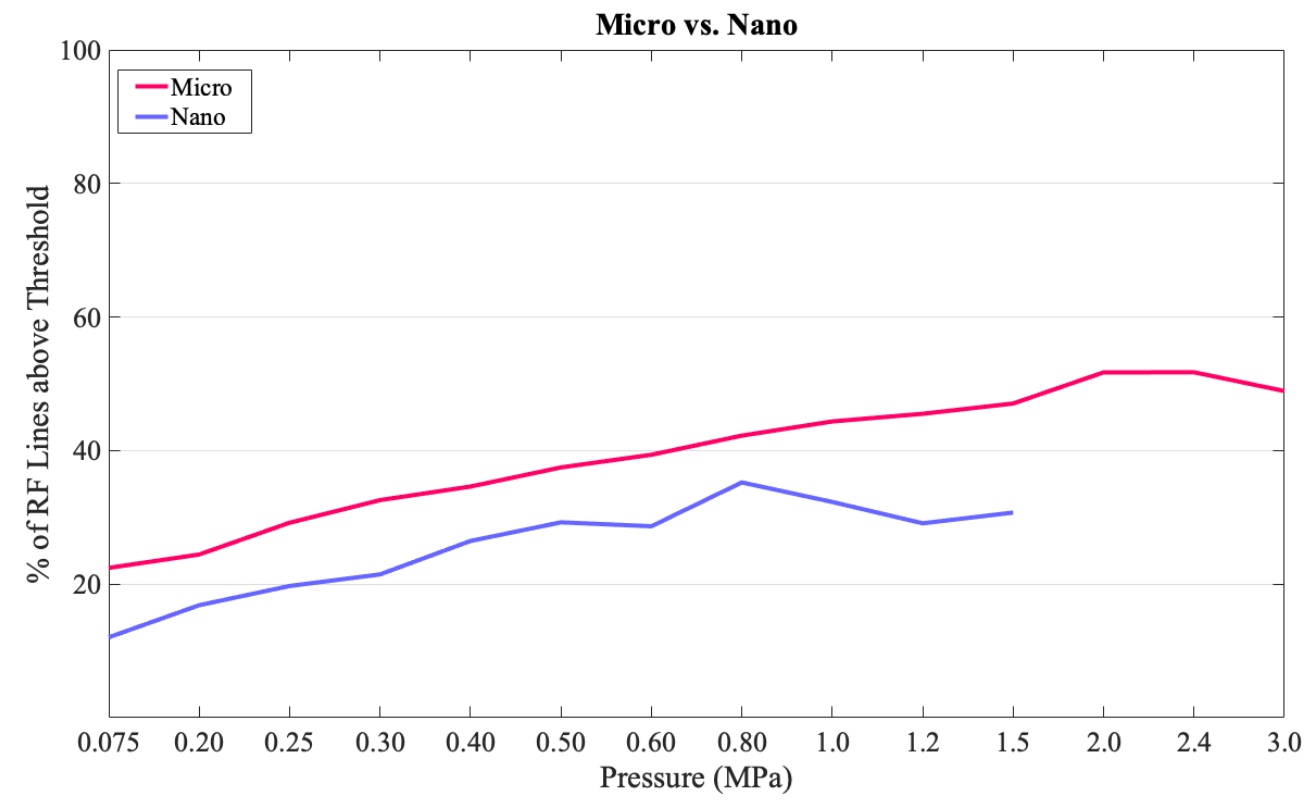

FIGURE 4.1: Percentage of signals that pass the initial criteria of a maximum power spectrum peak of greater than $0 \mathrm{~dB}$ in the algorithm for signal selection. This was averaged for all microbubbles and nanobubbles across both experiments.

\subsubsection{A Statistical Comparison of Microbubbles and Nanobubbles}

\section{Non-linear Behaviour of Microbubbles and Nanobubbles}

Average percentages were taken across all 5 shell types and compared for microbubbles and nanobubbles. Inspection of Figure 3.7 and Figure 3.8 shows a difference in 
the percentage of non-linear signals compared to the linear signals between the microbubble population and the nanobubble population. There are more microbubbles exhibiting nonlinear behaviour compared to nanobubbles. This is more clearly represented in Figure 4.3. This is consistent with the total count of non-linear signals shown in Figure 4.2 where it becomes evident there are not only more microbubbles signals that are behaving non-linearly, but also a higher percentage of total signals are undergoing non-linear oscillations.

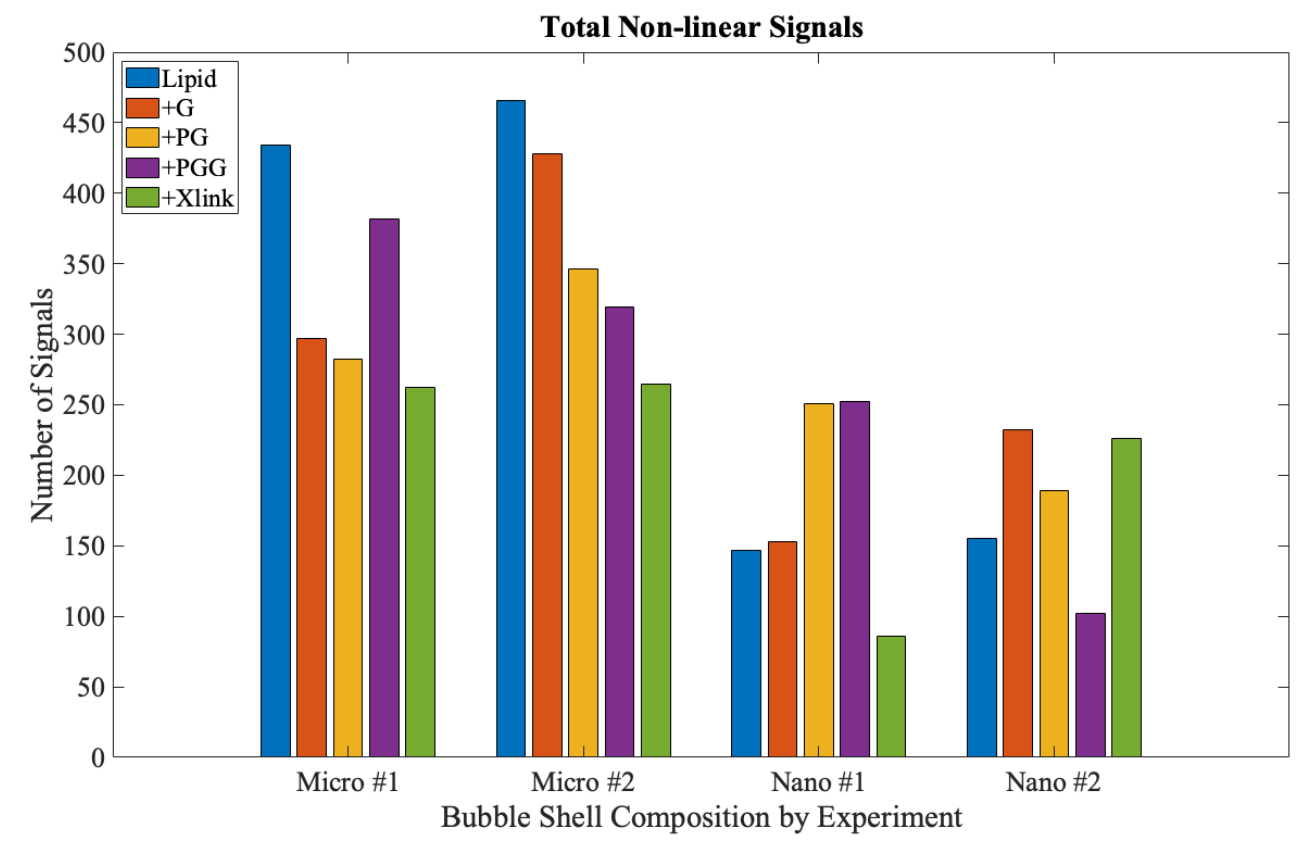

FIGURE 4.2: This is the total count of signals undergoing non-linear oscillations for each shell composition by experiment.

\section{Bubble Bursting}

The total number of burst signals for each type of shell and for each experiment are shown in Figure 4.4. Figure 4.5 shows the percentage of burst signals compared to all of the signals that pass the criteria described in subsection 2.4.1. There is a difference between the stability of the microbubbles compared to the nanobubbles for the frequency and pressures used in these experiments. No stable bubble signals were detected for nanobubbles exposed to pressures greater than $1500 \mathrm{kPa}$. 


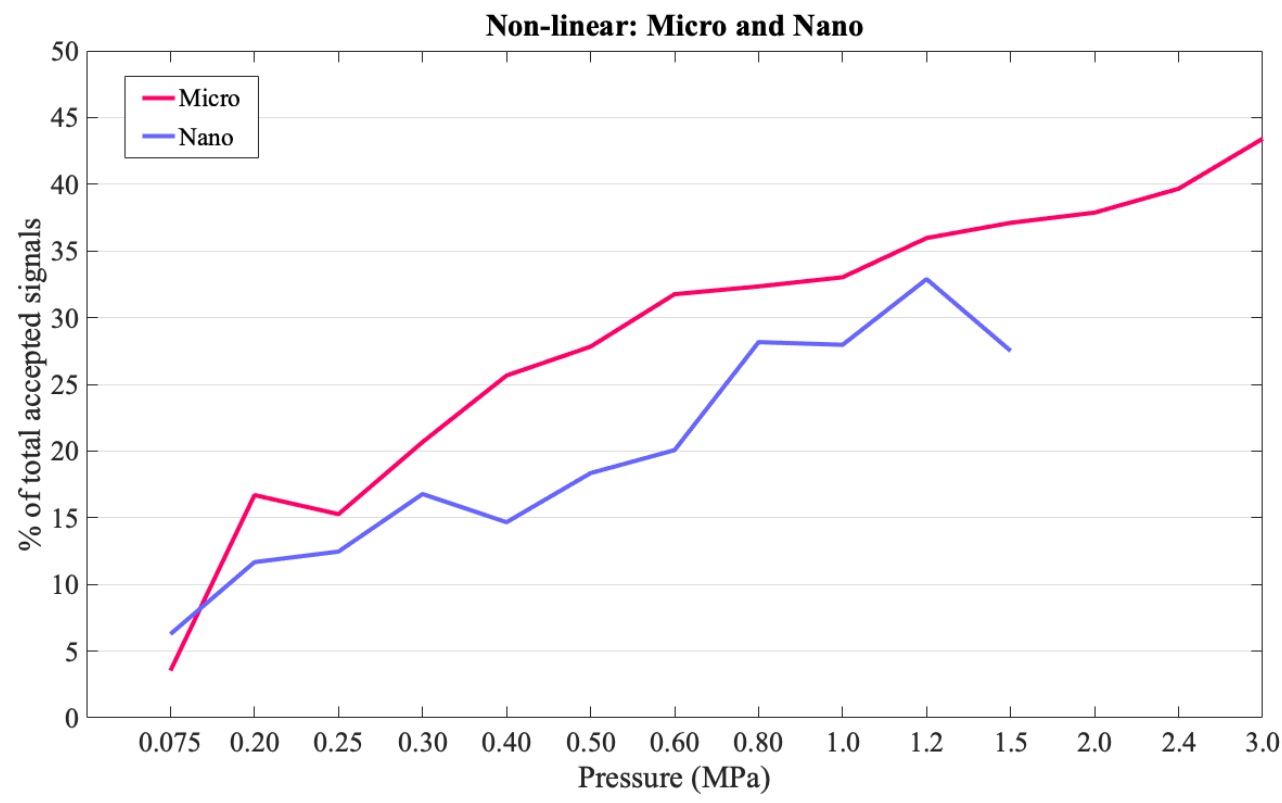

FIGURE 4.3: The percentage of signals undergoing non-linear behaviour from the total classified signals for microbubbles and nanobubbles.

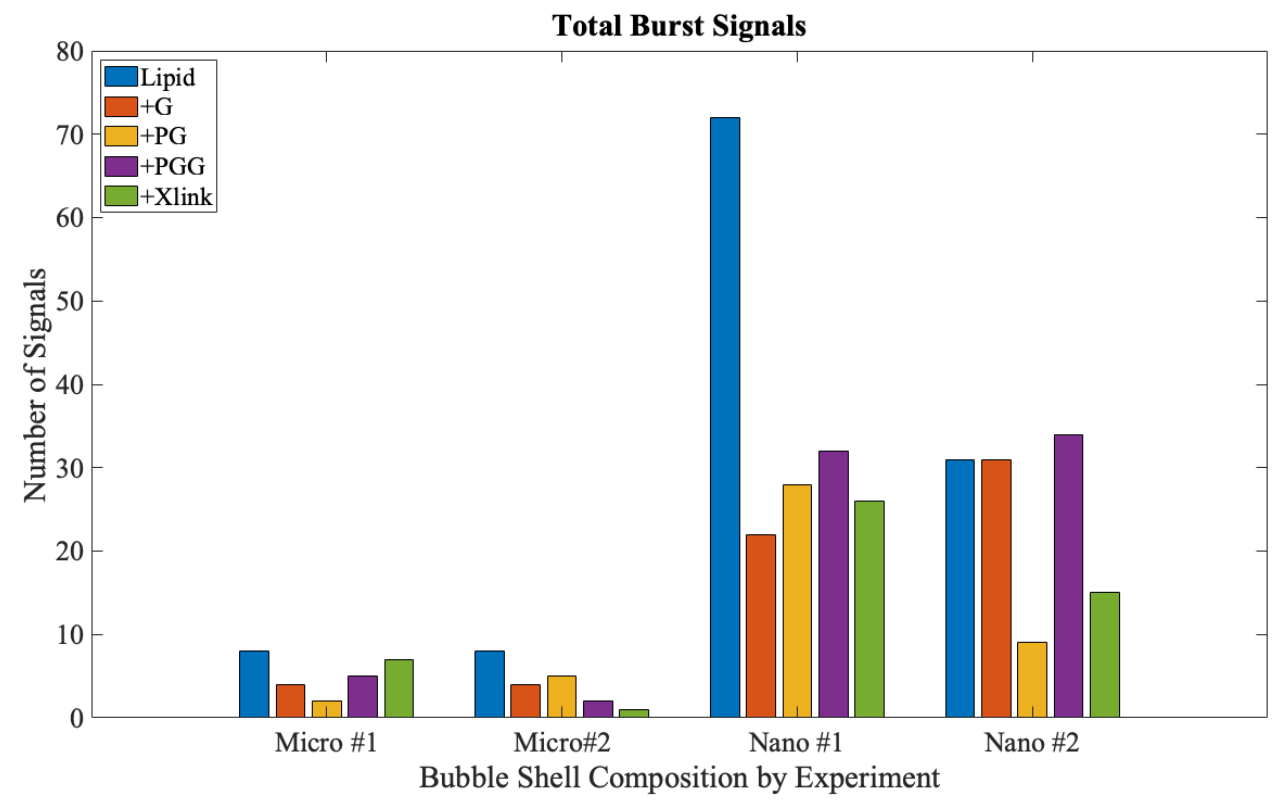

FIGURE 4.4: Total count of burst signals by each experiment. 


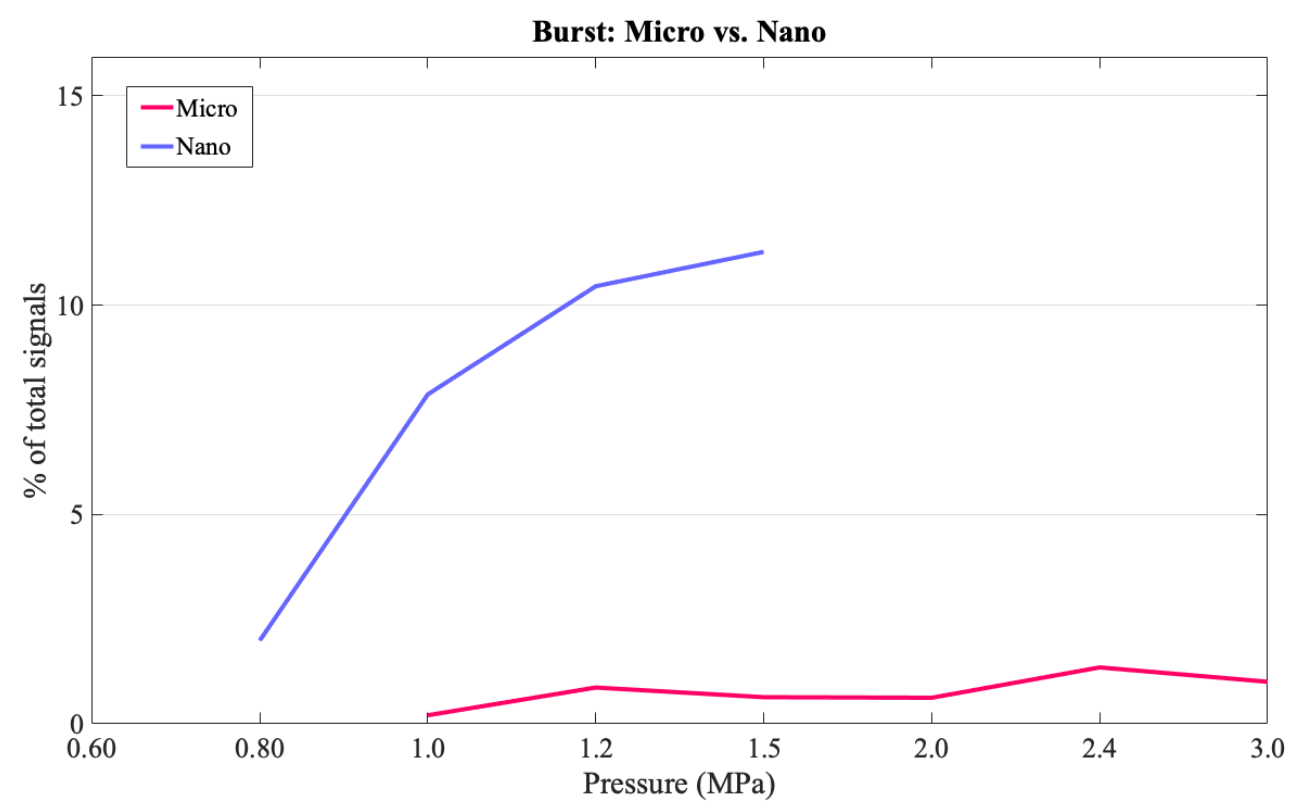

FIGURE 4.5: The percentage of total signals that have burst for microbubbles and nanobubbles.

As shown in figure 4.6, in bifurcation simulations performed by another graduate student in the laboratory, Amin J. Sojahrood, nanobubbles are more likely to undergo destruction, compared to microbubbles because nanobubbles have the largest p1 oscillation peak amplitude. The accepted destruction threshold for a bubble is $\frac{R \max }{R_{0}}<2$. Over this threshold the bubble is highly susceptible to destruction and under it destruction will be minimal [36].

\section{Period 3 Oscillations}

The number of $\mathrm{p} 3$ signals measured for microbubbles is much greater than the p3 signals measured for nanobubbles (which is closed to zero). The total counts of p3 signals observed over all pressures for each experiment are represented in Figure 4.7. The number of p3 signals counted for all samples of microbubbles are similar across all of the bubble shell types.

The abundance of $\mathrm{p} 3$ signals for microbubbles and the scarcity of p3 signals for 

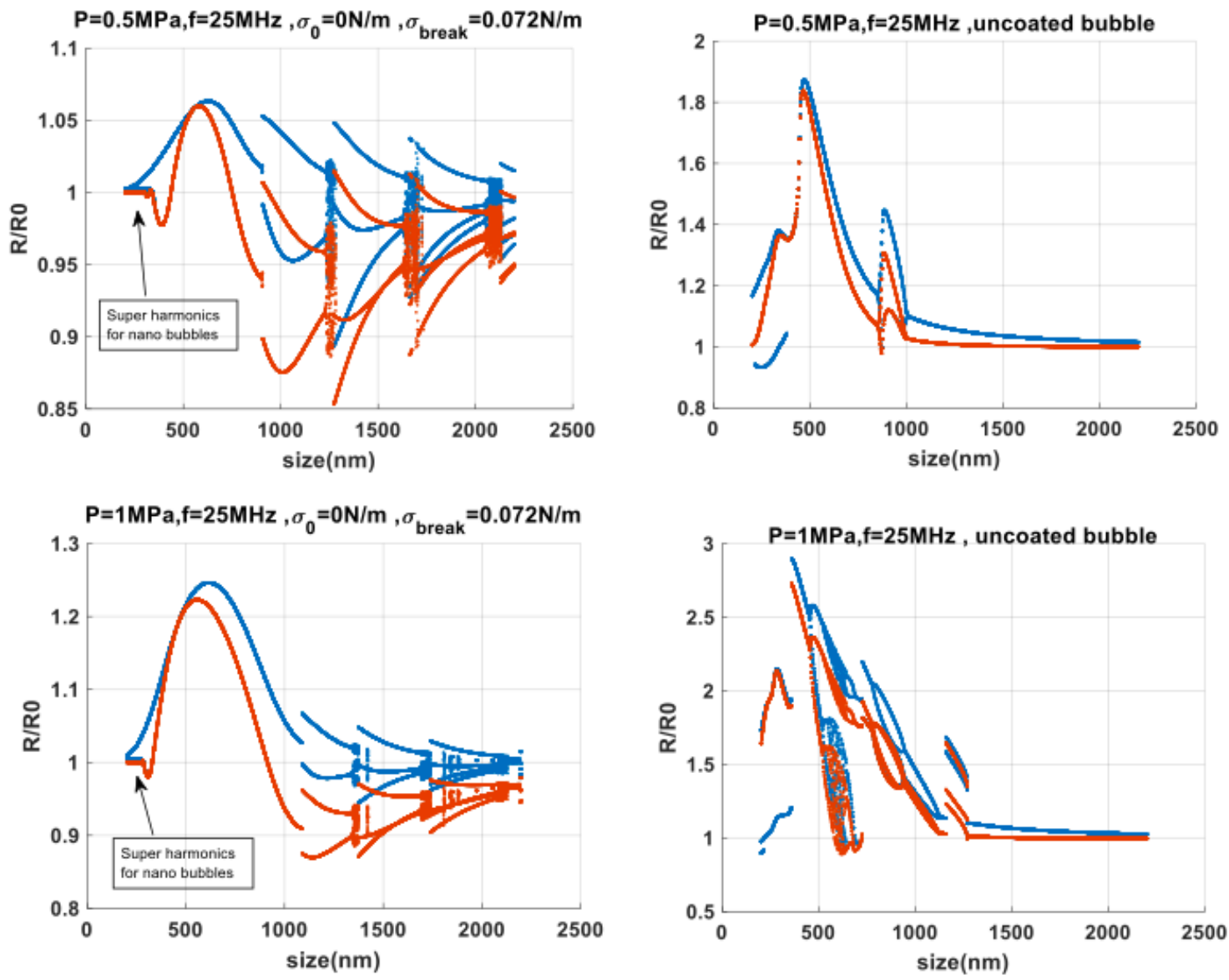

FIGURE 4.6: Bifurcation diagram of lipid and uncoated bubbles as a function of initial bubble size, where the initial surface tension is zero, at $25 \mathrm{MHz}$ for $500 \mathrm{kPa}$ (top) and $1 \mathrm{MPa}$ (bottom). The blue curve uses the method of maxima and the red curve uses the conventional method. 


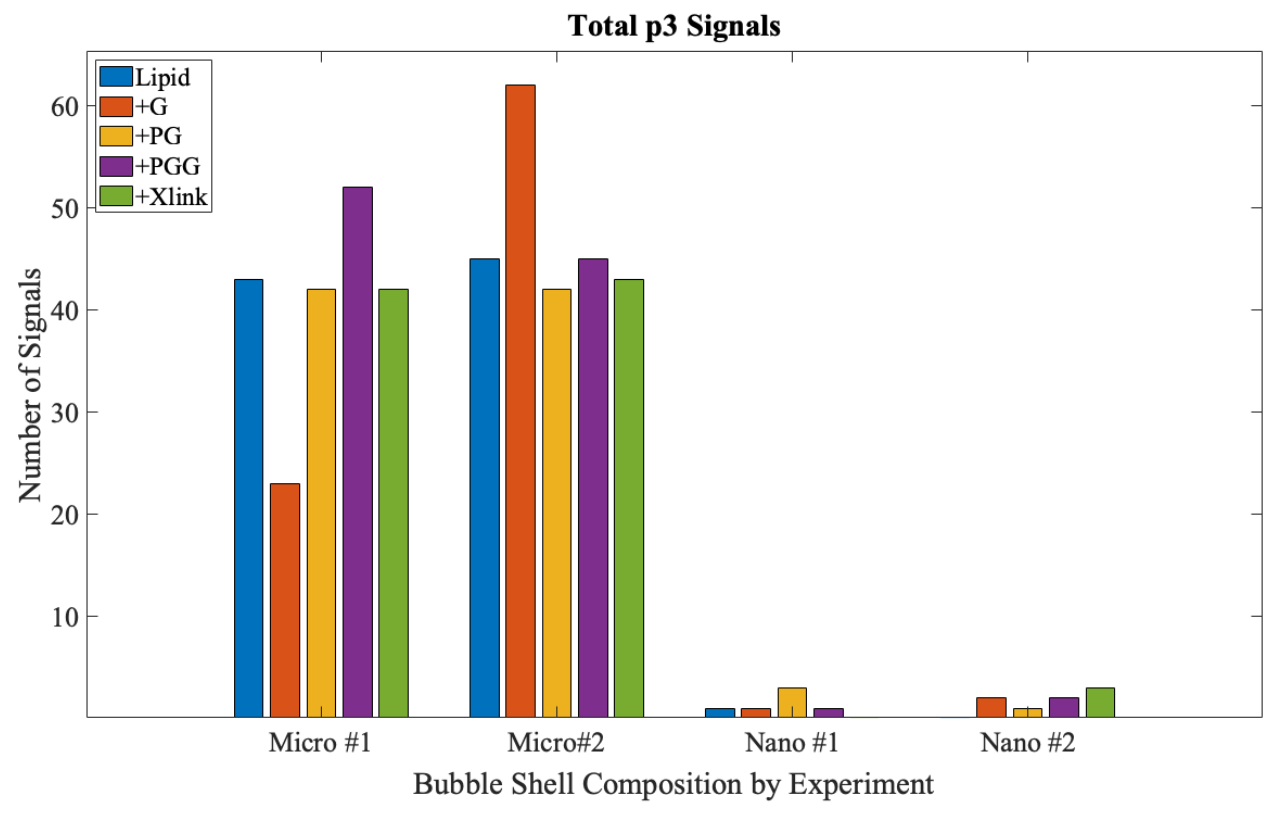

FIGURE 4.7: This is the total count of $\mathrm{p} 3$ signals for all experiments.

nanobubbles is related to how the bubbles respond to the US sonication frequencies and pressures. Simulations based on these exposure parameters are shown in Figure 4.6. Nanobubbles will mostly undergo p1 oscillations and some p2 oscillations, which is what was observed experimentally. Super-harmonic oscillations will occur but at frequencies that wouldn't have been detectable in these experiments considering the bandwidth limitations of the transducer with a centre frequency of $25 \mathrm{MHz}$ and $\sim 100 \%$ bandwidth ( $12.5 \mathrm{MHz}$ to 37.5 $\mathrm{MHz}$. Also, p3 oscillations will typically occur at approximately three times the resonant frequency of the bubble.

\subsubsection{Fundamental and Harmonic Amplitude Comparison}

No observable differences were discovered in the non-linear behaviour between the bubbles with different shells when comparing the amplitude of the harmonics compared to the fundamental. Differences between microbubbles and nanobubbles were observed in the analysis of the p2 fundamental and ultra harmonic amplitudes. These differences are 
displayed in Figure 4.8 which show the fundamental amplitude comparison between microbubbles and nanobubbles and also in Figure 4.9 which shows the ultra harmonic amplitude comparison. These is an increase in amplitude for the nanobubbles beginning at 500 $\mathrm{kPa}$ and continuing through to $1500 \mathrm{kPa}$ (the limit of the data collected for the nanobubble experiments due to increased destruction at higher pressures).

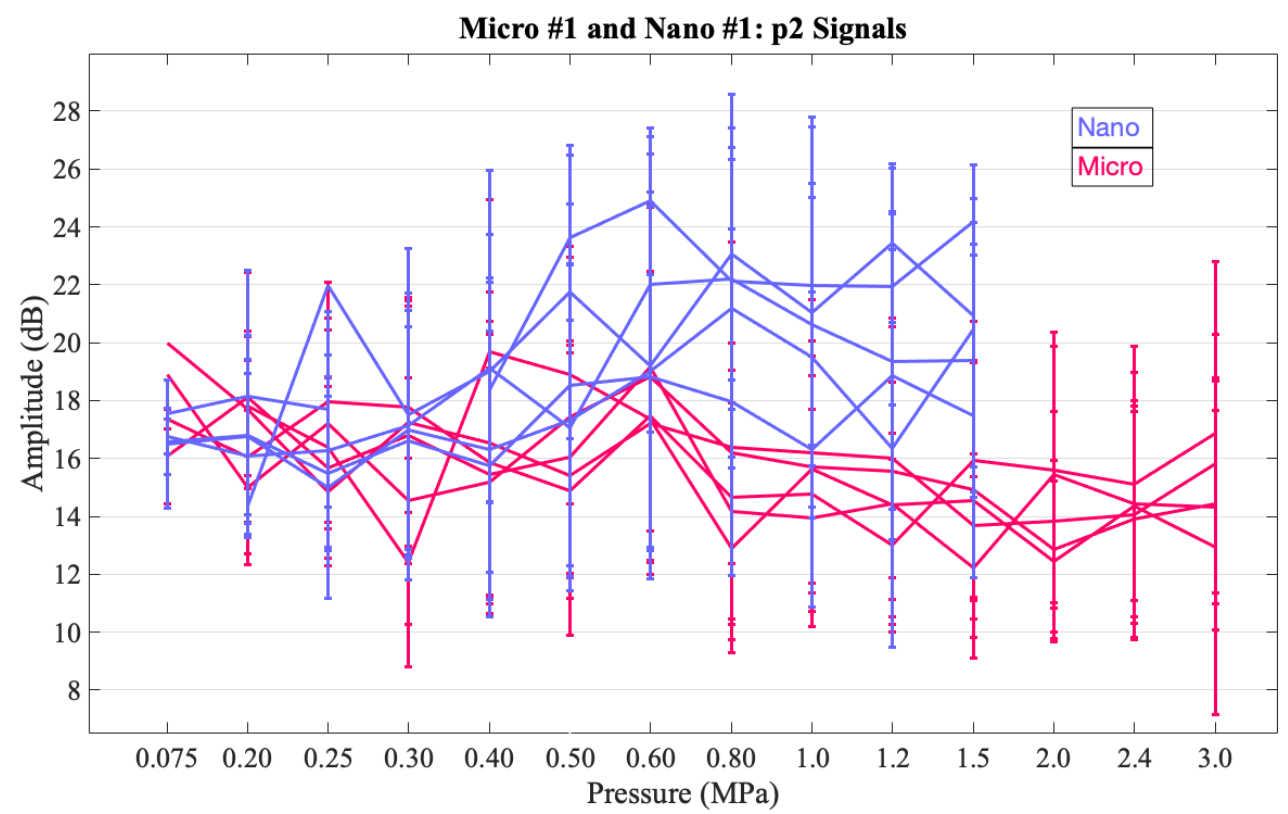

FIGURE 4.8: Average fundamental amplitude measured and averaged across microbubbles in experiment \#1 and nanobubbles in experiment \#1. 


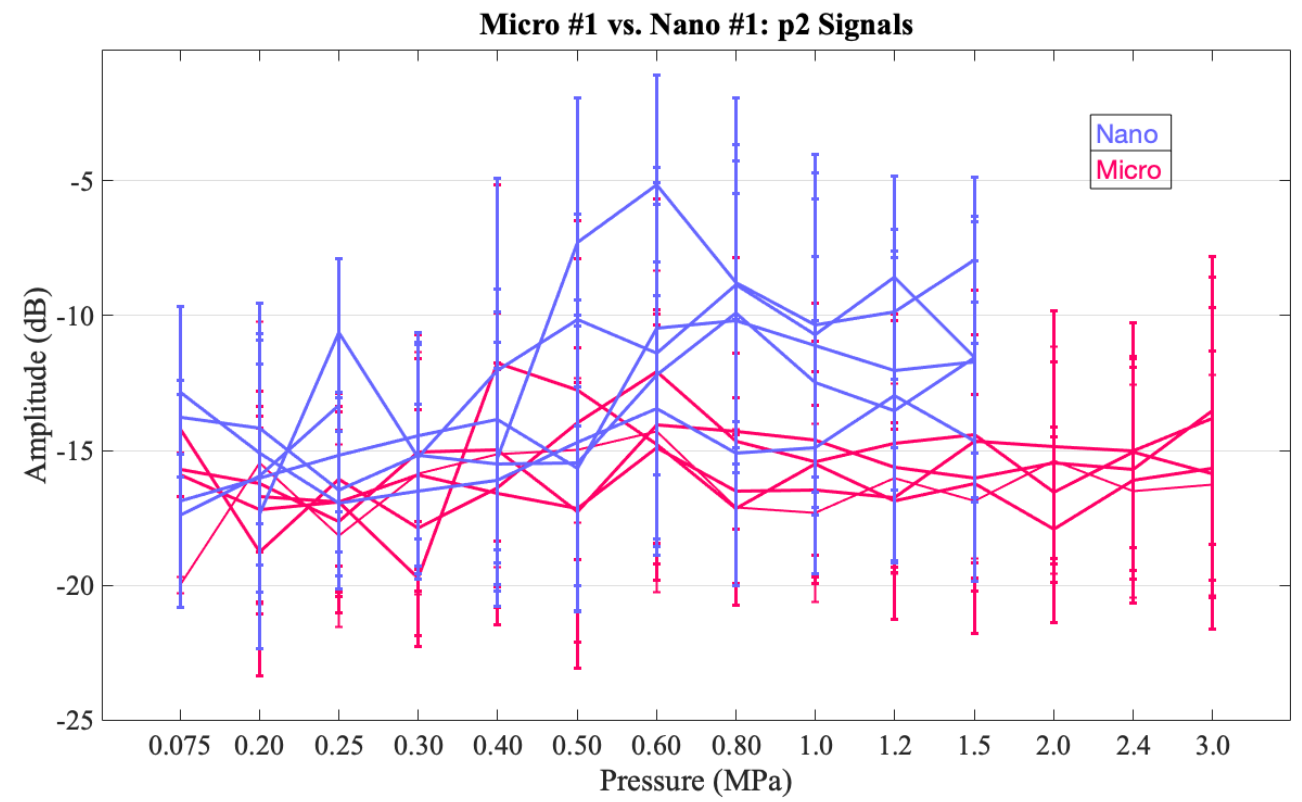

FIGURE 4.9: Average ultra harmonic amplitude measured and averaged across microbubbles in experiment \#1 and nanobubbles in experiment \#1.. 


\section{Chapter 5}

\section{Conclusion and Future Work}

\subsection{Conclusion}

The results show that for the given frequency and pressure range there is not an observable difference between the behaviour of the UCA's that were made with different shell components. Using the methods of analysis chosen, this conclusion is consistent for both the microbubble and nanobubble experiments. While the additive shell components that make up different bubbles have been shown under clinically relevant ultrasound parameters to have different stability in vivo, the results of this work based on an individual analysis of their oscillations does not show observable differences in their non-linear response to ultrasound. The polydispersity of the bubble size distribution and variation in shell uniformity could account for this.

The understanding of how an isolated microbubble or nanobubble behaves with different shell composition is vital to building a thorough understanding of how many microbubbles or nanobubbles with different shell compositions will behave under US.

Although there was not a trend between the different shell compositions, there is a difference between microbubbles and nanobubbles. Microbubbles and nanobubbles were compared using analysis that considered their non-linear behaviour, occurrence of p3 signals, threshold of destruction and fundamental and harmonic amplitudes of oscillation. 
It was determined that there are trends that differentiate microbubbles from nanobubbles in the single bubbles scattering experiments.

\subsection{Future Work}

The next step should be to create a narrower size distribution for the microbubbles and nanobubbles. As mentioned in the discussion, a more narrow size distribution with which to repeat these experiments with would provide greater sensitivity to how the shell compositions affect non-linear oscillatory behaviour of the bubbles. It is expected that results from a more monodisperse (same size) and homogeneous (shell thickness and composition uniformity) would reveal a greater difference in the pressure dependence of the scattering.

Additionally the experiments could be repeated with a different type of shell. All bubble shells used in this work were made with lipids. A more drastic change in shell composition would have a greater effect on the bubble behaviour analyzed through these methods. Furthermore, the gas inside the bubbles could be changed to determine how much of an effect it has on the compressibility of a bubble and its' non-linear behaviour. 


\section{Appendix A}

\section{Size Distribution Data}

\section{A.1 Microbubble Size Distribution}

This appendix contains the graphs which show the size distribution for the experiments done in this work. Figure A.1 shows the size distribution for all five types of shell compositions for Micro \#1 (the first experiments using microbubbles). Figure A.2 shows the size distribution for all five types of shell compositions for Micro \#2 (the repeat experiment for microbubbles). These graphs were acquired from size measurements done using the Beckman Coulter (Beckman Coulter, IN, USA).

\section{A.2 Nanobubble Size Distribution}

The nanobubble size distribution graphs were acquired from measurements done using Archimedes (Malvern Panalytical, MA, USA). Archimedes measures both buoyant (bubbles) and non-buoyant particles (micelles) which are both included in the graphs. Figure A.3 shows the size distribution for all five types of shell compositions for Nano \#1 (the first experiments using nanobubbles). Figure A.4 shows the size distribution for all five types of shell compositions for Nano \#2 (the repeat experiment for nanobubbles). 

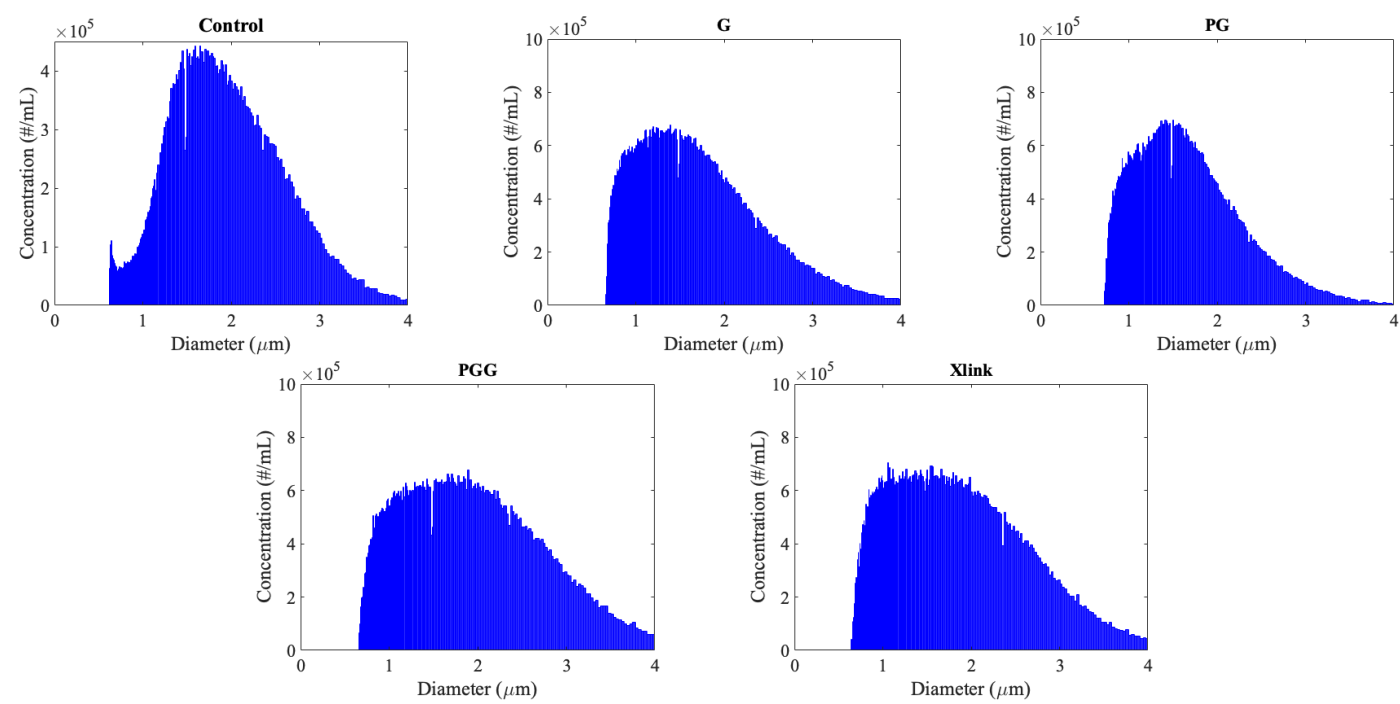

FIGURE A.1: Microbubble experiment \#1 size distribution graphs for each type of shell composition. These were acquired using the Beckman Coulter.
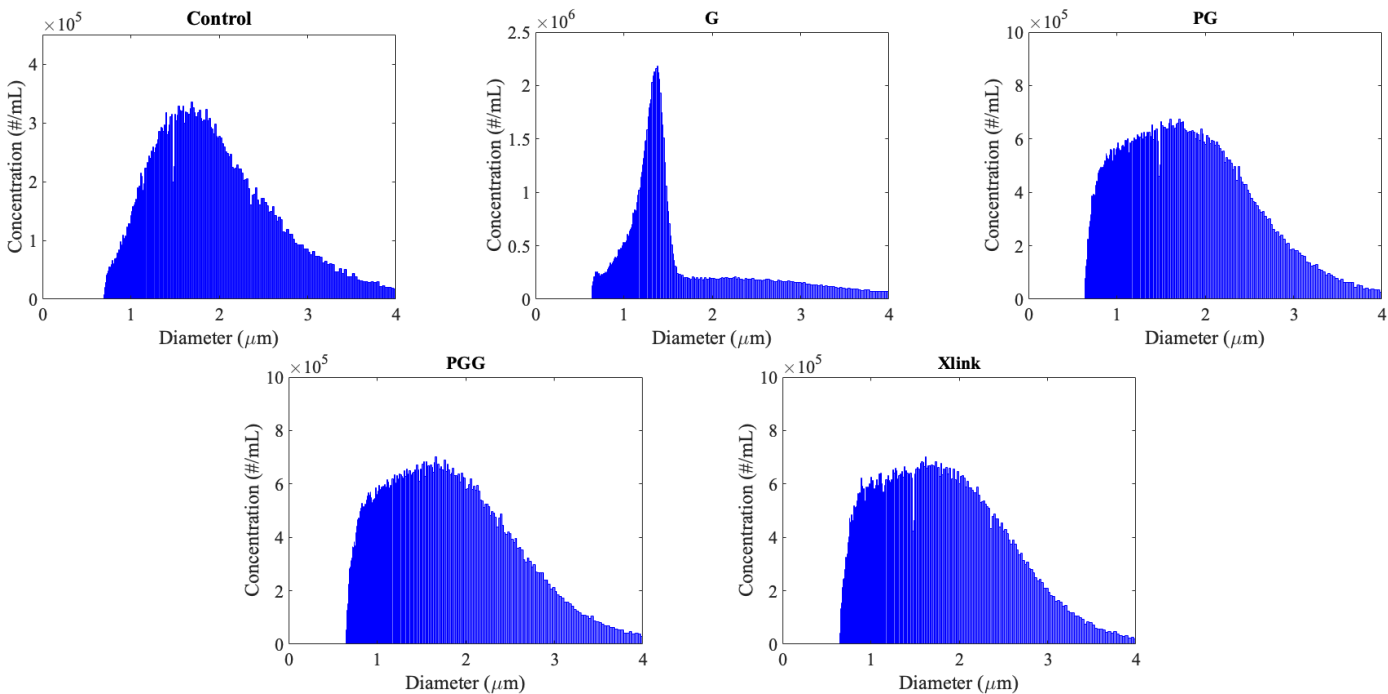

FIGURE A.2: Microbubble experiment \#2 size distribution graphs for each type of shell composition. These were acquired using the Beckman Coulter. 

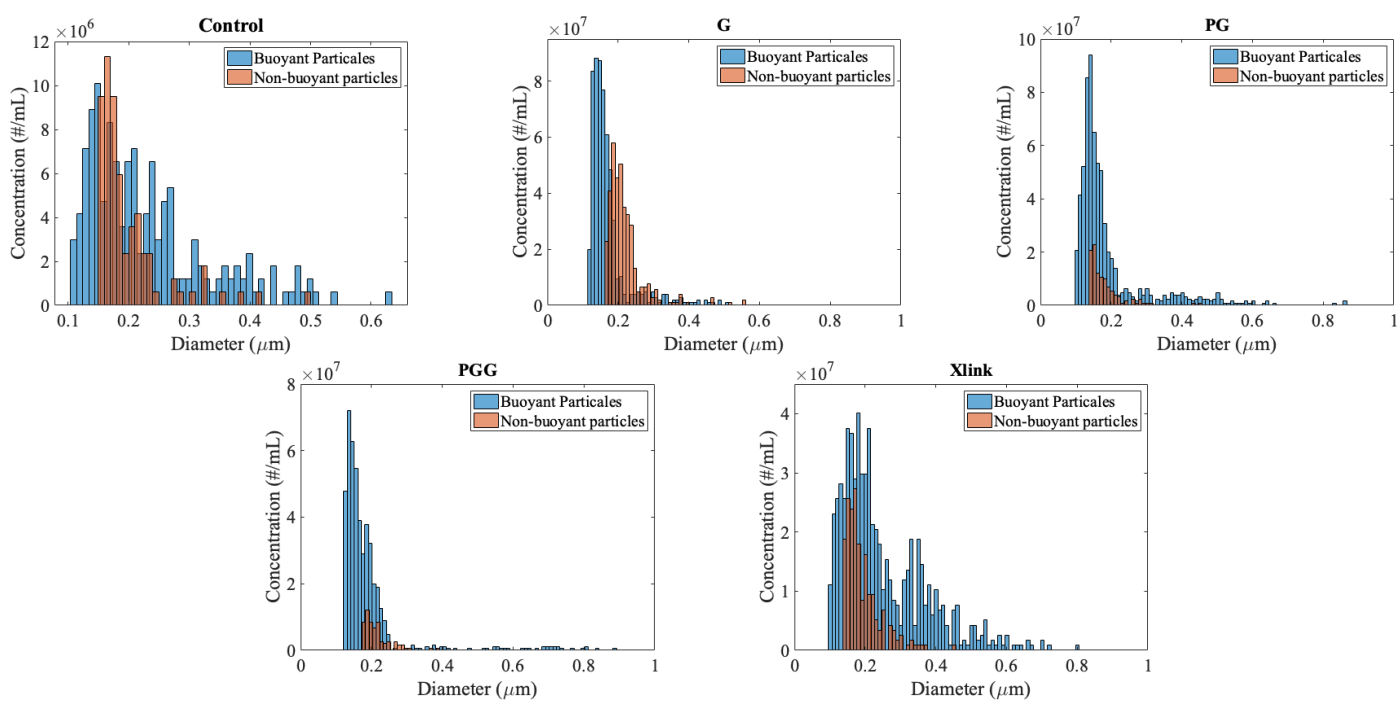

FIGURE A.3: Nanobubble experiment \#1 size distribution graphs for each type of shell composition. These were acquired using Archimedes.
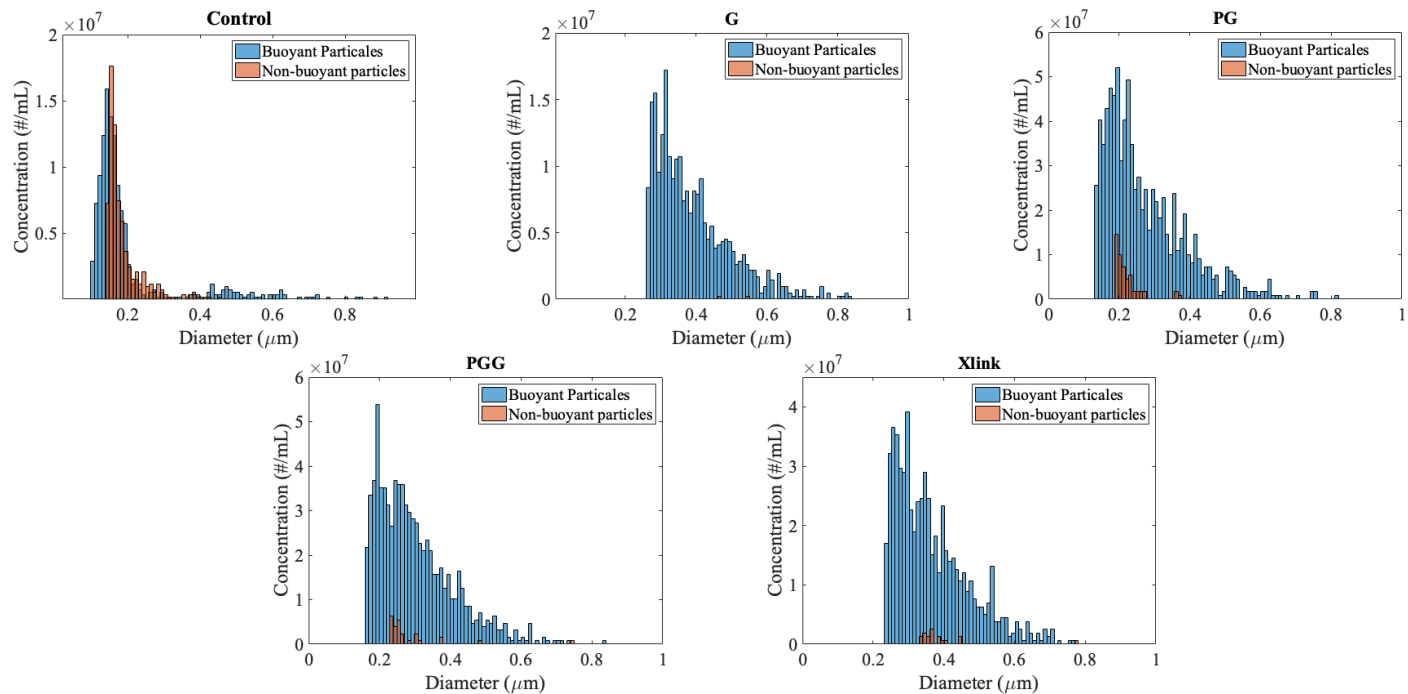

FIGURE A.4: Nanobubble experiment \#2 size distribution graphs for each type of shell composition. These were acquired using Archimedes 


\section{Appendix B}

\section{Additional Data}

\section{B.1 Count of Burst Signals}

The graphs that show, by experiment, the total number of burst signals for each shell composition type are shown for microbubbles in Figure B.1 and for nanobubbles in Figure B.2.

\section{B.2 Average Difference between the Fundamental and Harmonic Maximum Amplitudes}

The difference between the fundamental and subharmonic and the fundamental and the ultra harmonic maximum amplitudes calculated. They were then averaged across p2, p3, and p4 to determine if there were any difference evident across different non-linear signals. The graphs showing the fundamental and subharmonic amplitude difference can be seen in Figure B.3. The graphs showing the fundamental and ultra harmonic amplitude difference can be seen in Figure B.4. 

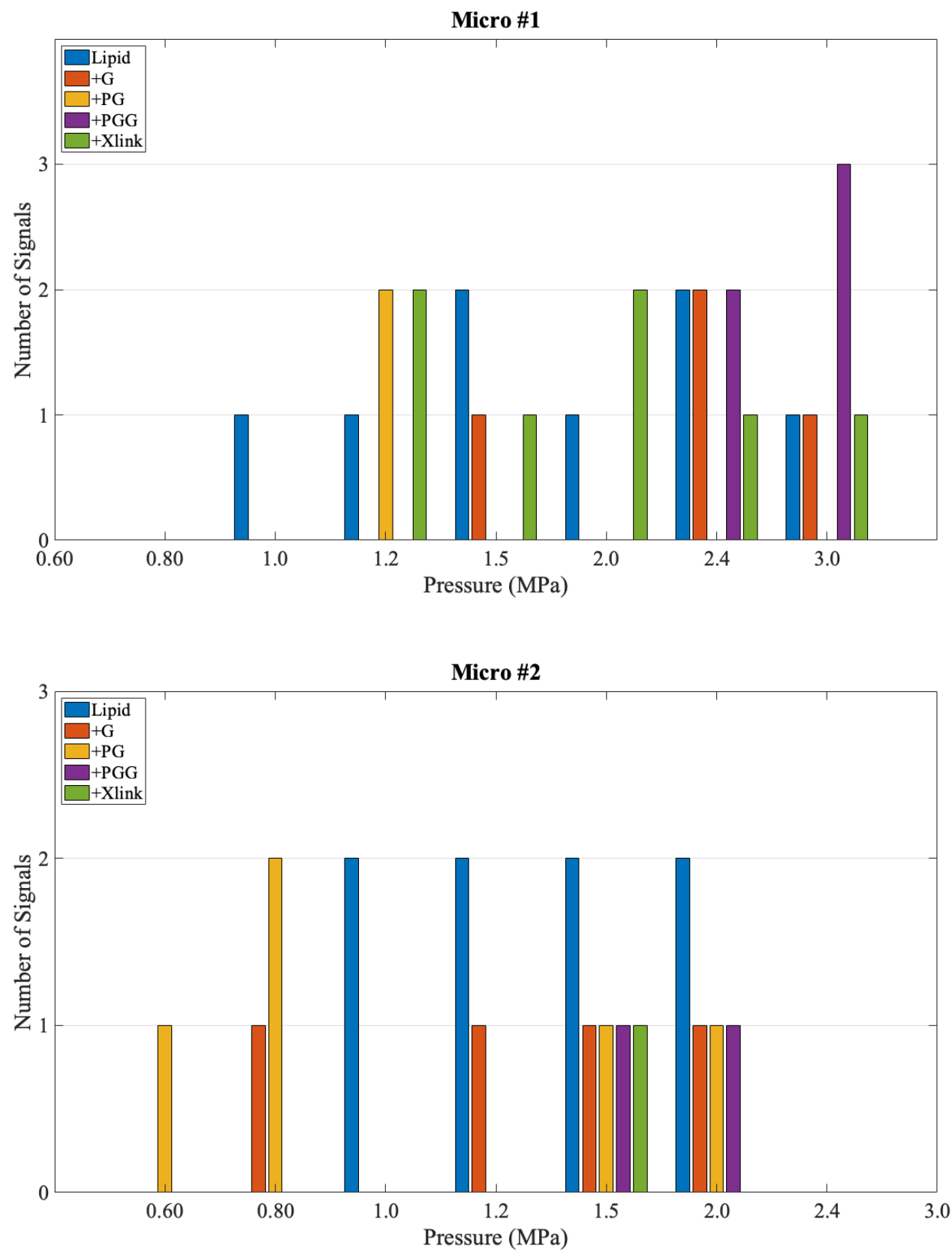

FIGURE B.1: Total number of burst signals for microbubbles in experiment \#1 (top) and experiment \#2 (bottom) across pressure. 

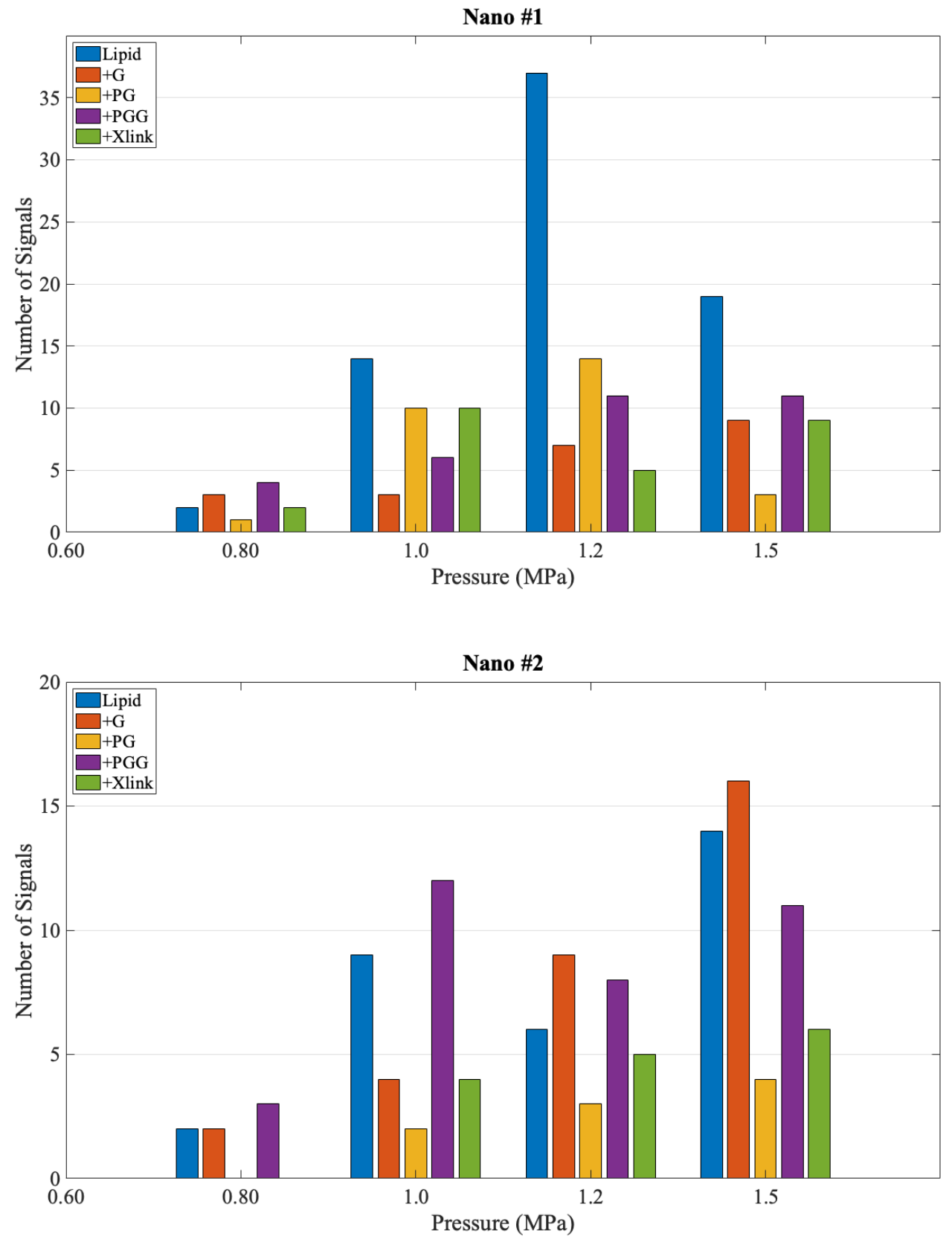

FIGURE B.2: Total number of burst signals nanobubbles in experiment \#1 (top) and experiment \#2 (bottom) across pressure. 

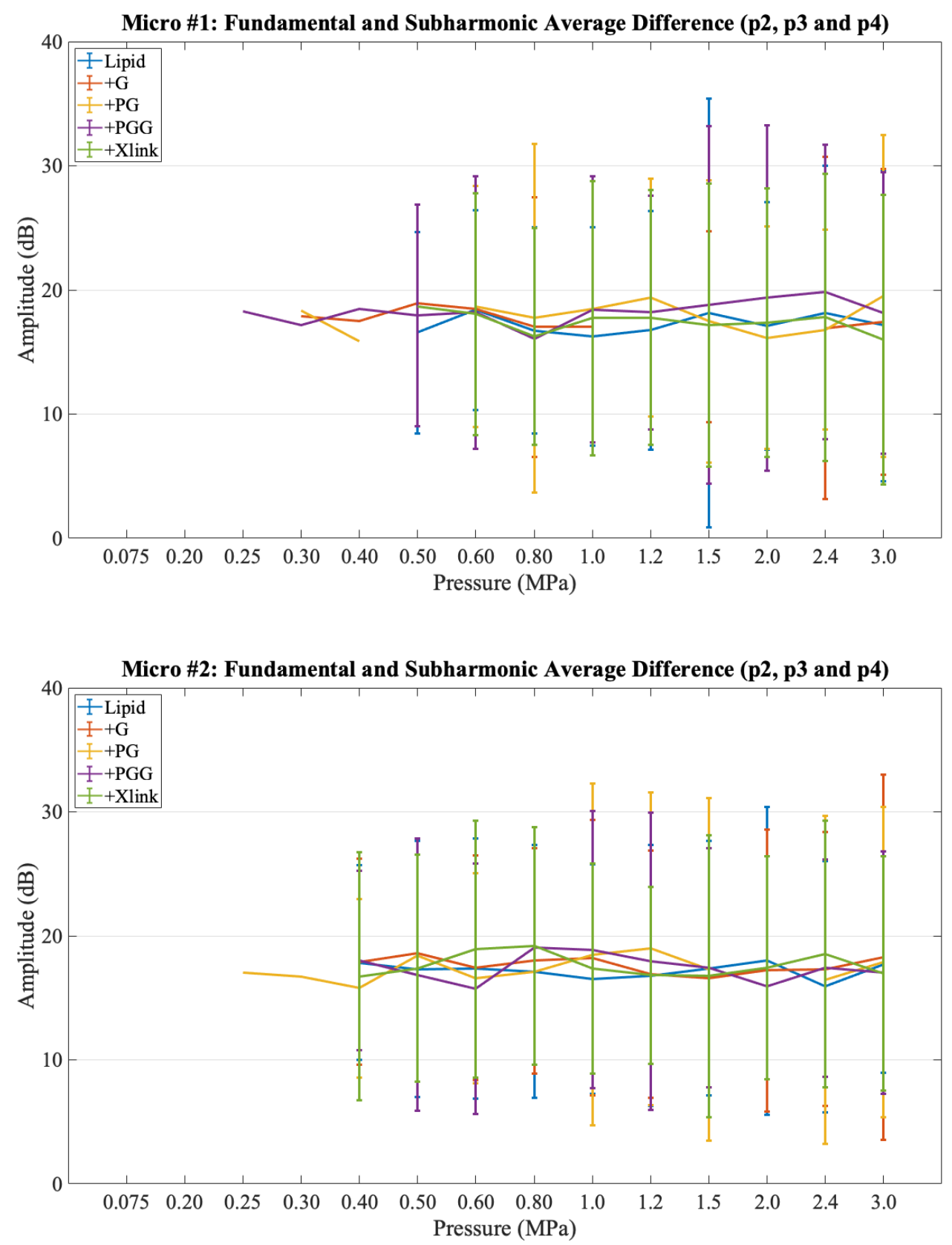

FIGURE B.3: Micro \#1 (top) and Micro \#2 (bottom) shows the difference between the fundamental and subharmonic amplitude maximum amplitudes averaged across p2, p3, and p4 signals. The error bars represent the standard deviation in calculating the mean for all signals across all pressures and signal

types. 

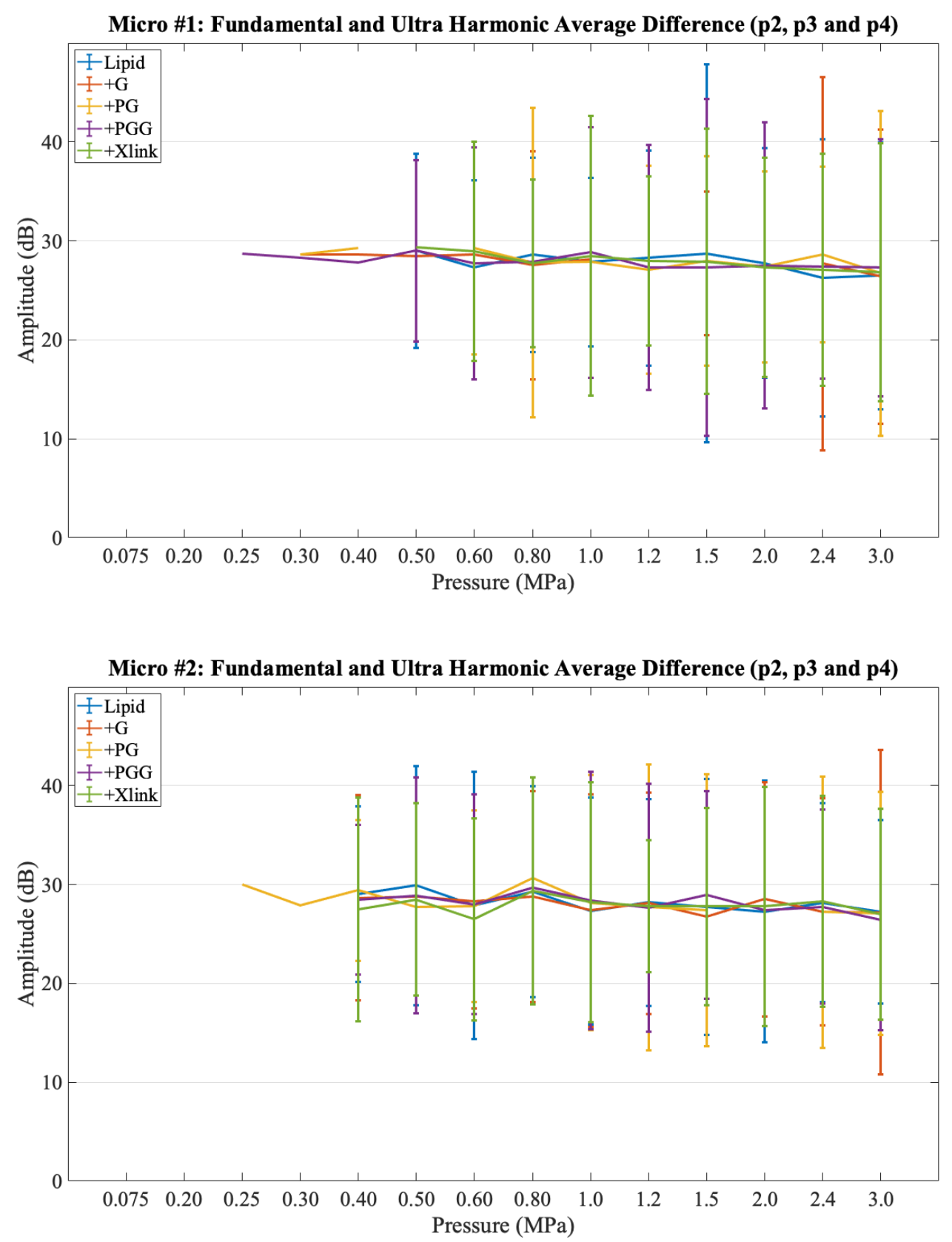

FIGURE B.4: Micro \#1 (top) and Micro \#2 (bottom) shows the difference between the fundamental and ultra harmonic amplitude maximum amplitudes averaged across p2, p3, and p4 signals. The error bars represent the standard deviation in calculating the mean for all signals across all pressures and signal

types. 


\section{Appendix C}

\section{MATLAB Codes}

\section{C.1 Extract Code}

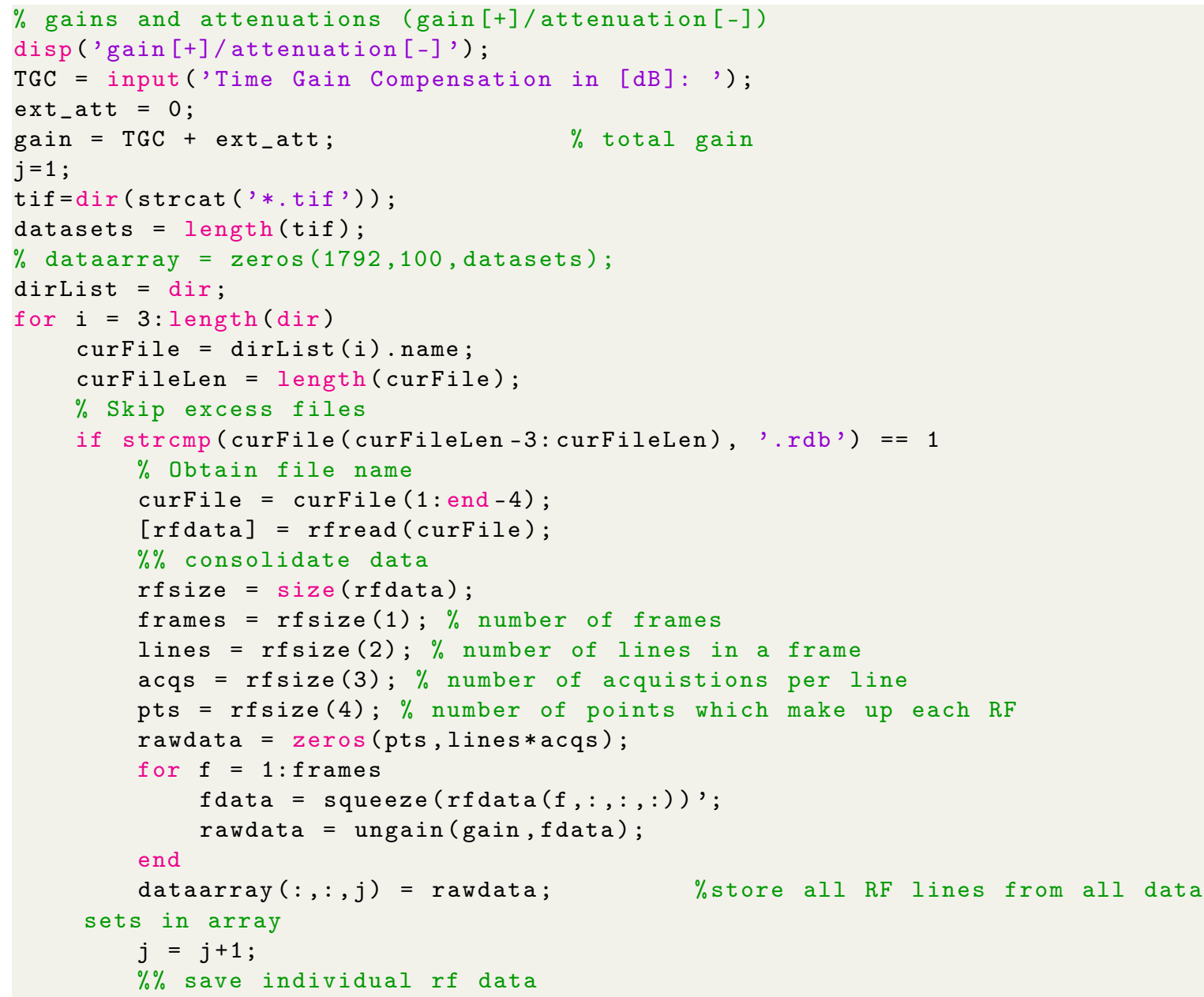


Appendix C. MATLAB Codes

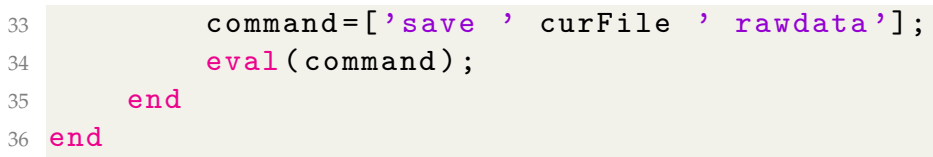

Listing C.1: Extract Code 


\section{C.2 Noise Threshold Code}

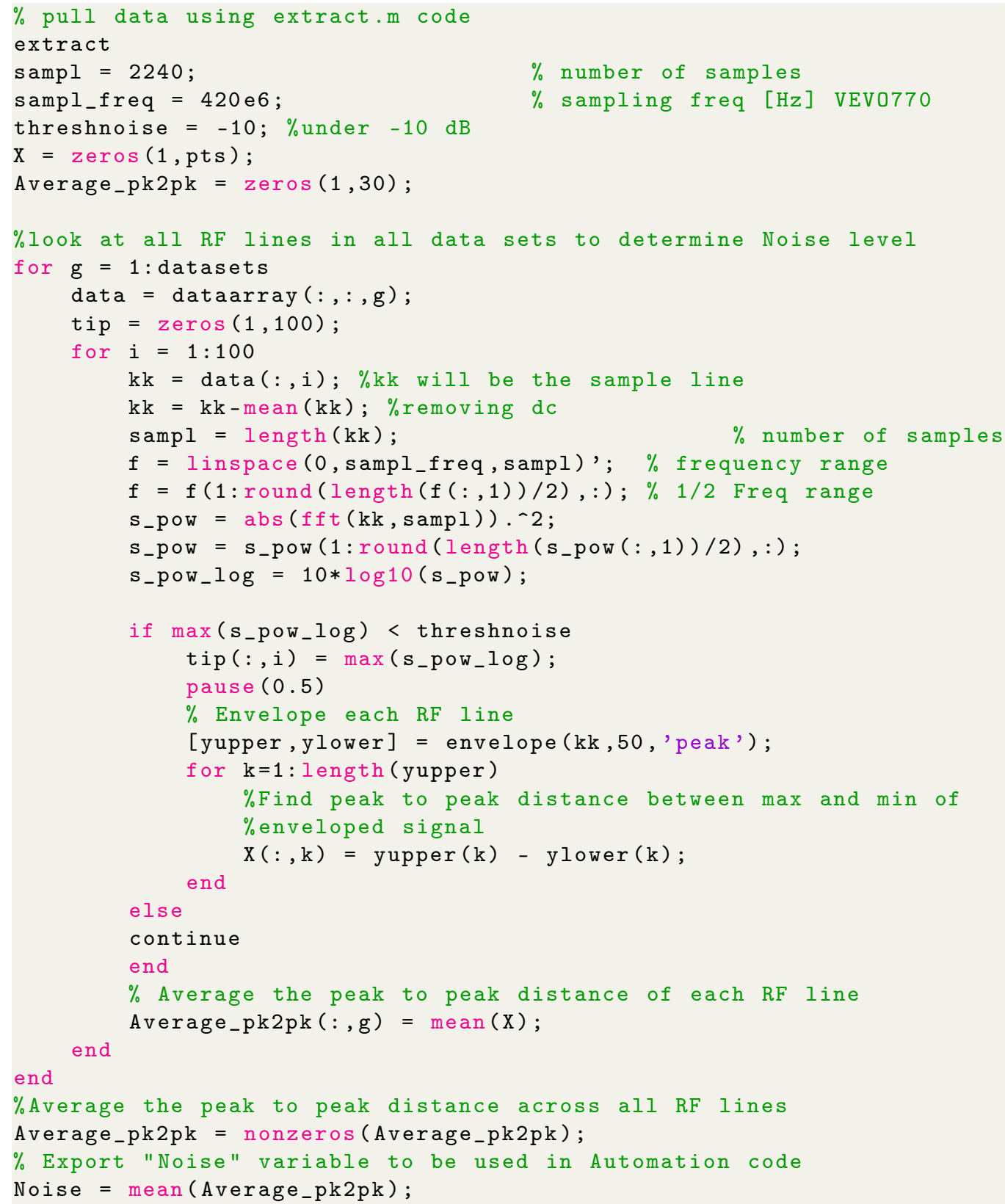

Listing C.2: Noise Threshold Code 


\section{C.3 Automation Code}

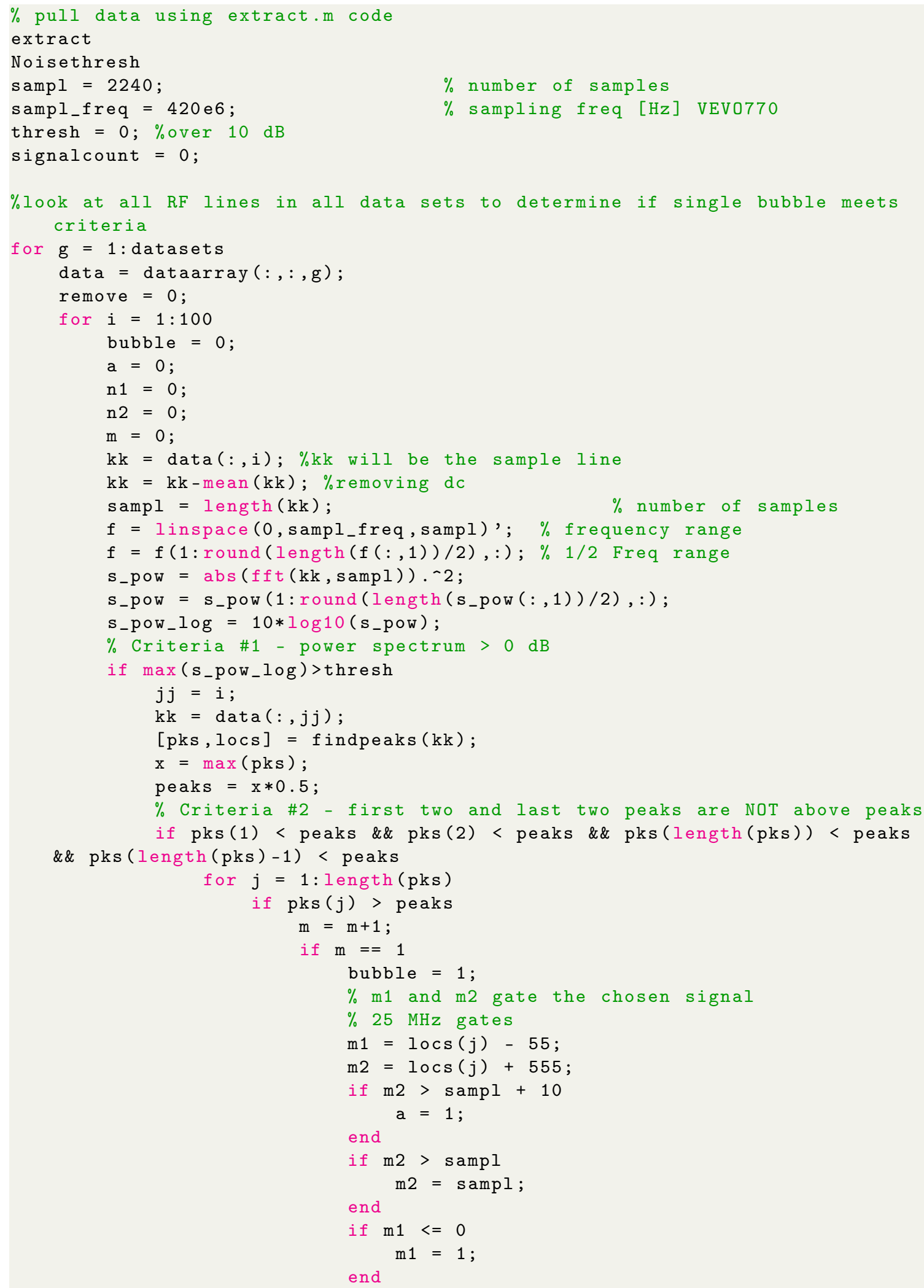


Appendix C. MATLAB Codes

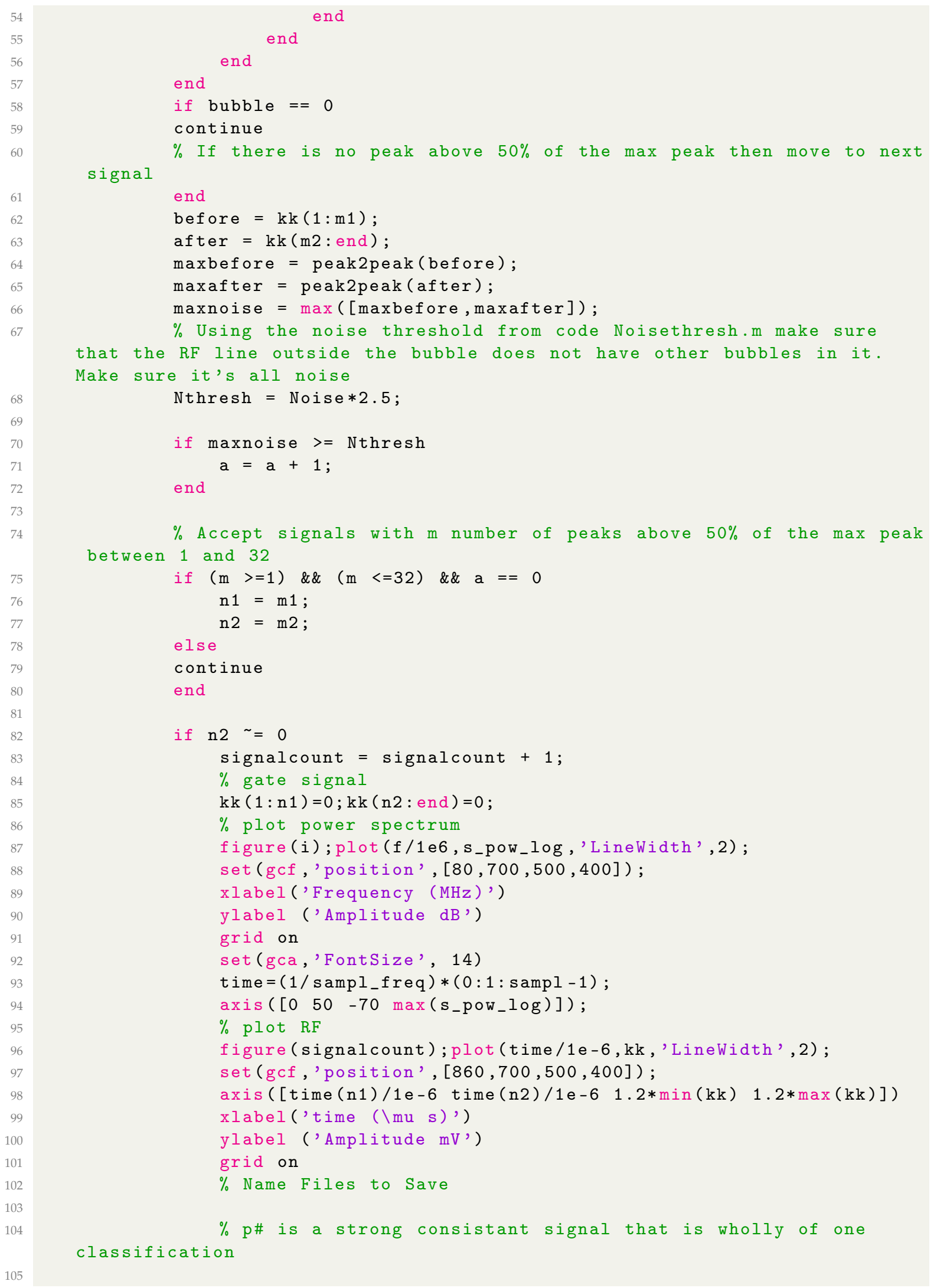




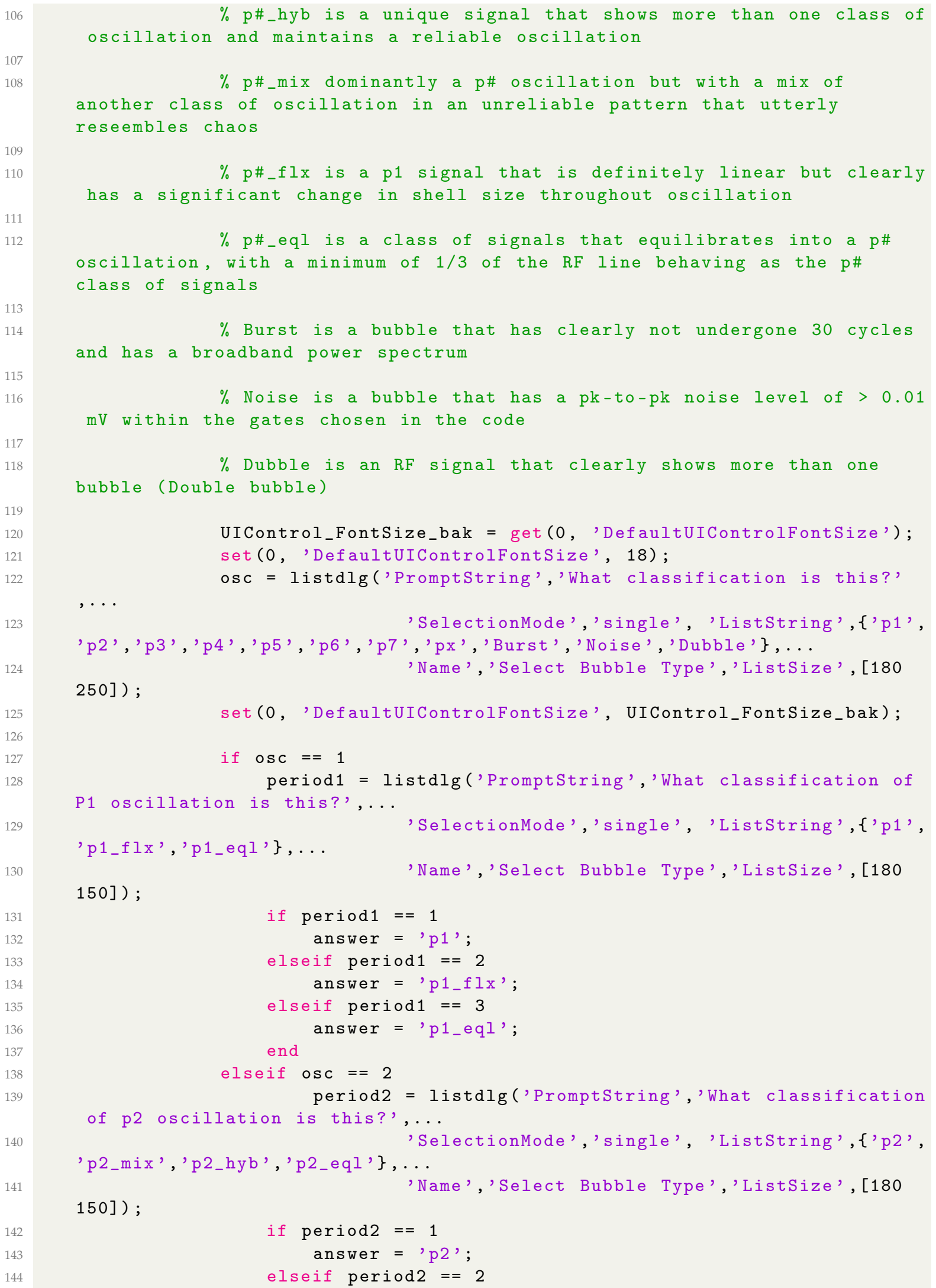




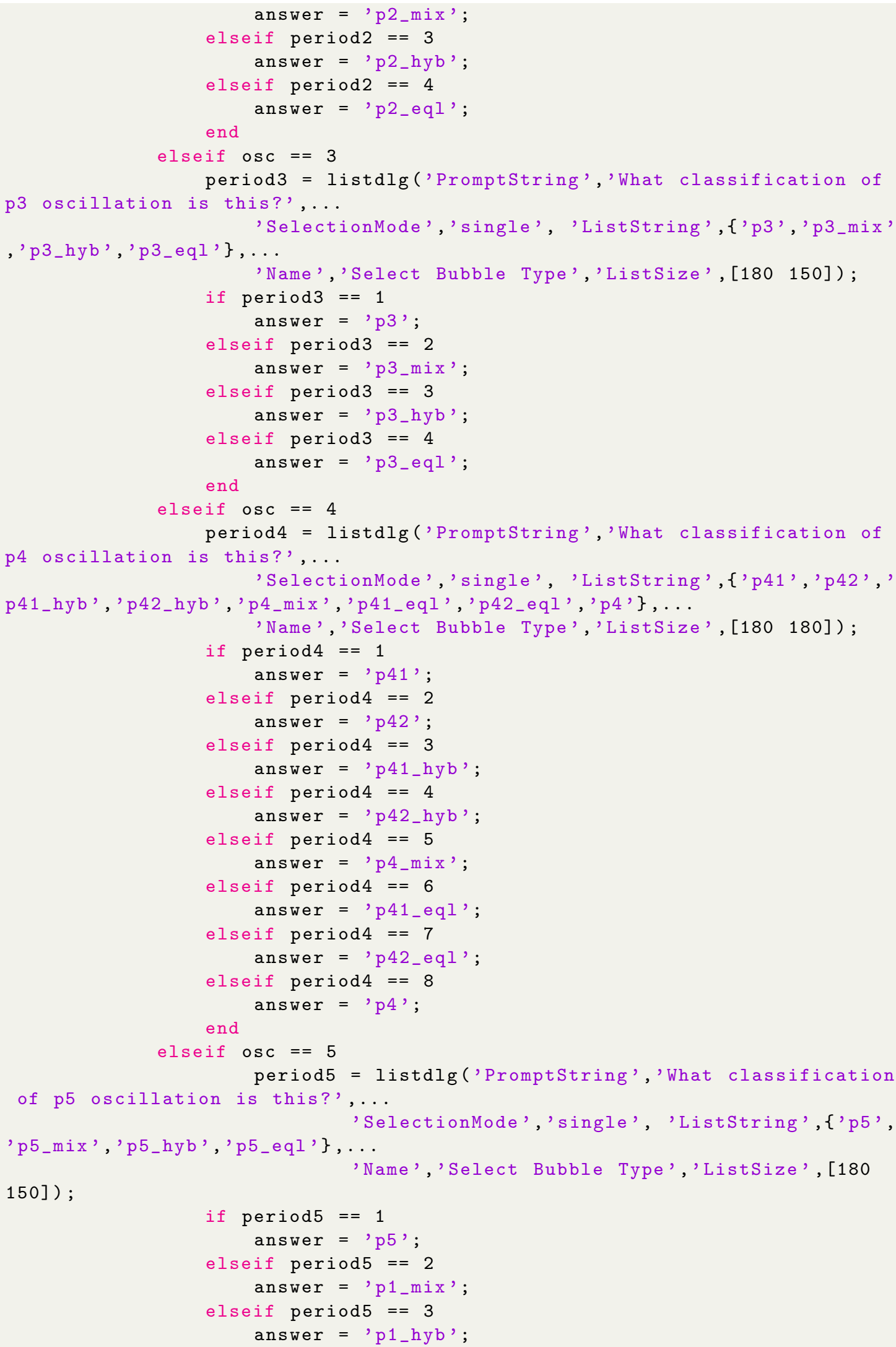




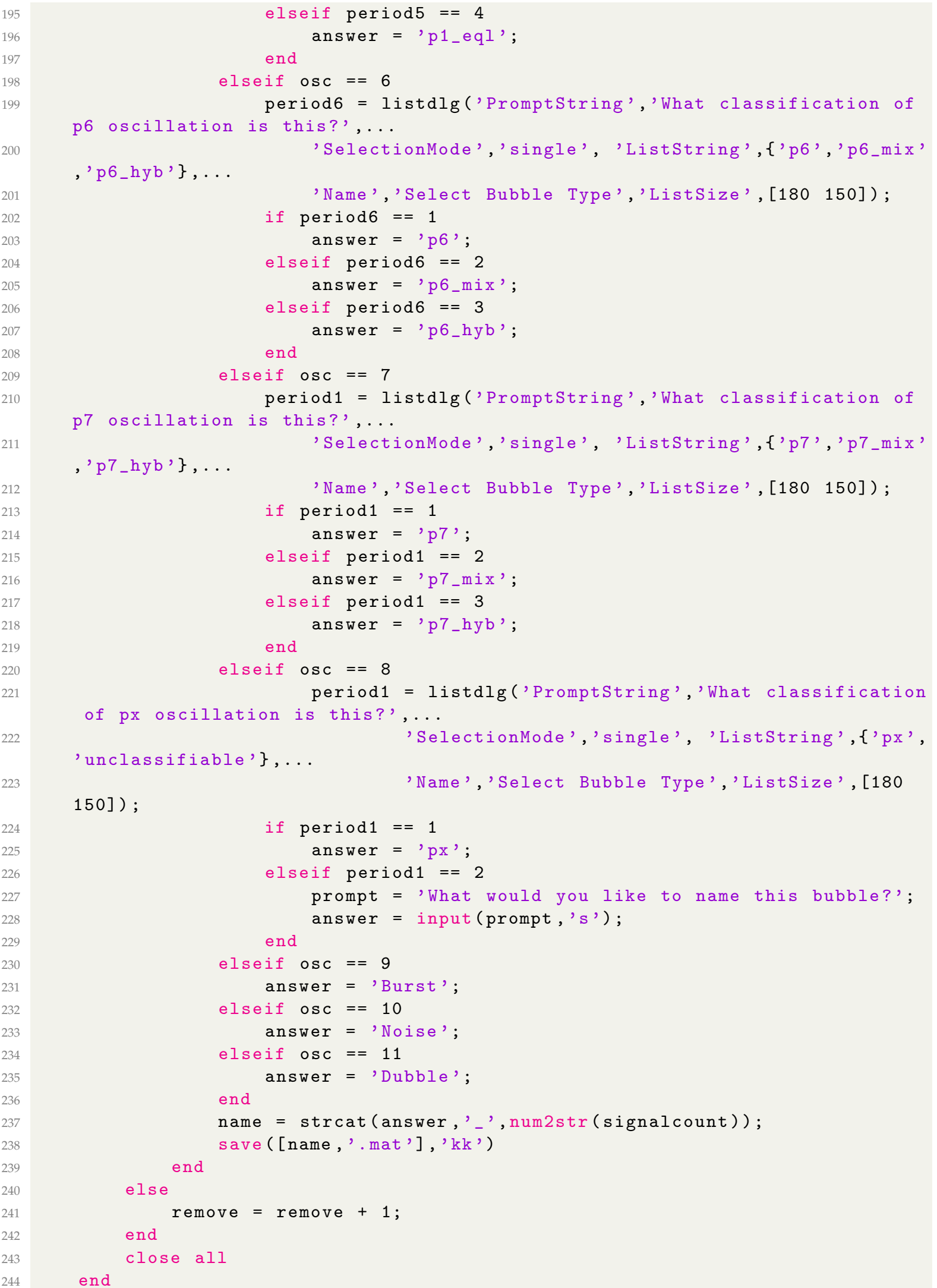


Appendix C. MATLAB Codes

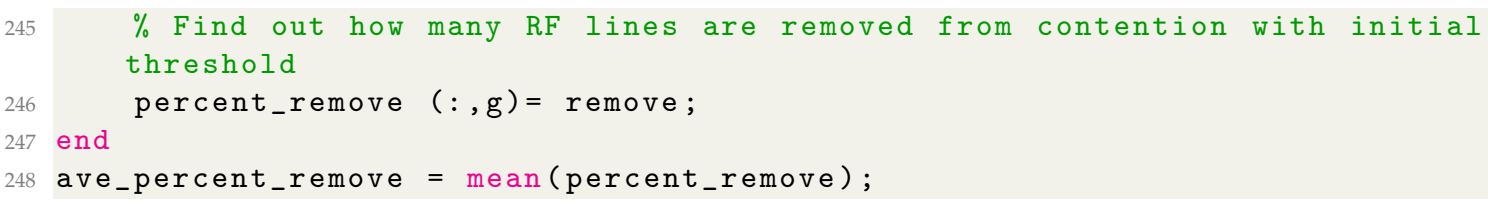

LISTING C.3: Automation Code 


\section{Bibliography}

[1] R. S. C. Cobbold, "Foundations of Biomedical Ultrasound", Oxford University Press, 2007.

[2] T. L. Szabo, Diagnostic Ultrasound Imaging: Inside Out: Second Edition. 2004, ISBN: 9780123964878. DOI: $10.1016 /$ C2011-0-07261-7.

[3] H. Azhari, Basics of Biomedical Ultrasound for Engineers. Hoboken, NJ, USA: John Wiley \& Sons, Inc., 2010, ISBN: 9780470561478. DOI: 10.1002/9780470561478.

[4] M. A. Borden and K. H. Song, "Reverse engineering the ultrasound contrast agent", Advances in Colloid and Interface Science, vol. 262, pp. 39-49, 2018, ISSN: 00018686. DOI: $10.1016 / j$.cis. 2018.10.004.

[5] N. C. Nanda, R. Gramiak, and P. M. Shah, "Diagnosis of aortic root dissection by echocardiography", Circulation, 1973, ISSN: 00097322. DOI: 10.1161/01 . CIR . 48 . 3. 506.

[6] N. De Jong, A. Bouakaz, and P. Frinking, "Basic acoustic properties of microbubbles", Echocardiography, 2002, ISSN: 07422822. DOI: 10.1046/j .1540-8175.2002.00229.x.

[7] E. Stride and N. Saffari, "On the destruction of microbubble ultrasound contrast agents", Ultrasound in Medicine and Biology, 2003, ISSN: 03015629. DOI: 10.1016/S0301-5629 (02) 00787-1.

[8] M. Emmer, The Onset of Bubble Vibration. 2009, pp. 1-176, ISBN: 9789090238289.

[9] S. R. Sirsi and M. A. Borden, "Microbubble compositions, properties and biomedical applications", Bubble Science, Engineering \& Technology, vol. 1, no. 1-2, pp. 3-17, 2009, ISSN: 1758-8960. DOI: $10.1179 / 175889709 \times 446507$.

[10] S. R. Sirsi and M. A. Borden, "Advances in ultrasound mediated gene therapy using microbubble contrast agents", Theranostics, 2012, ISSN: 18387640. DOI: 10.7150/thno. 4306.

[11] T. Segers, N. de Jong, D. Lohse, and M. Versluis, “Chapter 5. Microbubbles for Medical Applications", in Microfluidics for Medical Applications, 2015, pp. 81-101, ISBN: 978-184973-637-4. DOI: 10.1039/9781849737593-00081.

[12] R. H. Perera, C. Hernandez, H. Zhou, P. Kota, A. Burke, and A. A. Exner, "Ultrasound imaging beyond the vasculature with new generation contrast agents", Wiley Interdisciplinary Reviews: Nanomedicine and Nanobiotechnology, 2015, ISSN: 19390041. DOI: 10.1002/wnan.1326.

[13] W. T. Shi and F. Forsberg, "Ultrasonic characterization of the nonlinear properties of contrast microbubbles", Ultrasound in Medicine and Biology, vol. 26, no. 1, pp. 93-104, 2000, ISSN: 03015629. DOI: 10.1016/S0301-5629(99) 00117-9. 
[14] C. J. Harvey, M. J. Blomley, R. J. Eckersley, R. A. Heckemann, J. Butler-Barnes, and D. O. Cosgrove, "Pulse-inversion mode imaging of liver specific microbubbles: Improved detection of subcentimetre metastases", Lancet, vol. 355, no. 9206, pp. 807808, 2000, ISSN: 01406736. DOI: 10.1016/S0140-6736 (99) 04545-6.

[15] P. Qin, T. Han, A. C. Yu, and L. Xu, "Mechanistic understanding the bioeffects of ultrasound-driven microbubbles to enhance macromolecule delivery", Journal of Controlled Release, vol. 272, no. January, pp. 169-181, 2018, ISSN: 18734995. DOI: 10.1016/ j.jconrel.2018.01.001.

[16] K. H. Martin and P. A. Dayton, "Current status and prospects for microbubbles in ultrasound theranostics", Wiley Interdisciplinary Reviews: Nanomedicine and Nanobiotechnology, 2013, ISSN: 19395116. DOI: 10.1002/wnan. 1219.

[17] S. Qin, C. F. Caskey, and K. W. Ferrara, "Ultrasound contrast microbubbles in imaging and therapy: Physical principles and engineering", Physics in Medicine and Biology, 2009, ISSN: 00319155. DOI: 10.1088/0031-9155/54/6/R01.

[18] J. Park, D. Park, U. Shin, S. Moon, C. Kim, H. S. Kim, H. Park, K. Choi, B. K. Jung, J. Oh, and J. Seo, "Synthesis of laboratory ultrasound contrast agents", Molecules, vol. 18, no. 10 , pp. 13 078-13 095, 2013, ISSN: 14203049. DOI: $10.3390 / \mathrm{molecules} 181013078$.

[19] L. T. G., "Derivation of the Rayleigh-Plesset Equation in Terms of Volume", Tech. Rep., 2013. DOI: $10.1017 /$ CB09781107415324.004. arXiv: arXiv: 1011.1669v3.

[20] P. Marmottant, S. van der Meer, M. Emmer, M. Versluis, N. de Jong, S. Hilgenfeldt, and D. Lohse, "A model for large amplitude oscillations of coated bubbles accounting for buckling and rupture", The Journal of the Acoustical Society of America, vol. 118, no. 6, pp. 3499-3505, 2005, ISSN: 0001-4966. DOI: 10.1121/1.2109427.

[21] R. C. Tolman, "The effect of droplet size on surface tension", The Journal of Chemical Physics, 1949, ISSN: 00219606. DOI: 10.1063/1.1747247.

[22] P.-L. Lin, R. J. Eckersley, and E. A. H. Hall, "Ultrabubble: A Laminated Ultrasound Contrast Agent with Narrow Size Range", Advanced Materials, vol. 21, pp. 3949-3952, 2009, ISSN: 09359648. DOI: 10 . 1002/adma. 200901096.

[23] E. G. Schutt, D. H. Klein, R. M. Mattrey, and J. G. Riess, Injectable microbubbles as contrast agents for diagnostic ultrasound imaging: The key role of perfluorochemicals, 2003. DOI: 10.1002/anie. 200200550.

[24] A. De Leon, R. Perera, C. Hernandez, M. Cooley, O. Jung, S. Jeganathan, E. Abenojar, G. Fishbein, A. Jafari Sojahrood, C. Emerson, P. L. Stewart, M. Kolios, and A. Exner, "Contrast Enhanced Ultrasound Imaging by Nature-Inspired Ultrastable Echogenic Nanobubbles", Nanoscale, 2019, ISSN: 2040-3364. DOI: 10.1039/c9nr04828f.

[25] G. M. M. El Maghraby, A. C. Williams, and B. W. Barry, "Interactions of surfactants (edge activators) and skin penetration enhancers with liposomes", International Journal of Pharmaceutics, vol. 276, no. 1-2, pp. 143-161, 2004, ISSN: 03785173. DOI: 10.1016/ j.ijpharm. 2004.02.024.

[26] R. H. Abou-Saleh, J. R. McLaughlan, R. J. Bushby, B. R. Johnson, S. Freear, S. D. Evans, and N. H. Thomson, "Molecular Effects of Glycerol on Lipid Monolayers at the Gas, ÄiLiquid Interface: Impact on Microbubble Physical and Mechanical Properties", Langmuir, 2019, ISSN: 0743-7463. DOI: 10.1021/acs . langmuir.8b04130.

[27] R. H. Perera, H. Wu, P. Peiris, C. Hernandez, A. Burke, H. Zhang, and A. A. Exner, "Physics of bubble oscillations", The Journal of the Acoustical Society of America, vol. 73, no. 2, p. 106501, 2018, ISSN: 0001-4966. DOI: 10.1088/0034-4885/73/10/106501. 
[28] H. Wu, N. G. Rognin, T. M. Krupka, L. Solorio, H. Yoshiara, G. Guenette, C. Sanders, N. Kamiyama, and A. A. Exner, "Acoustic characterization and pharmacokinetic analyses of new nanobubble ultrasound contrast agents", Ultrasound in Medicine and Biology, 2013, ISSN: 1879291X. DOI: 10.1016/j .ultrasmedbio. 2013.05.007.

[29] H. Mulvana, R. J. Browning, Y. Luan, N. De Jong, M. X. Tang, R. J. Eckersley, and E. Stride, "Characterization of contrast agent microbubbles for ultrasound imaging and therapy research", IEEE Transactions on Ultrasonics, Ferroelectrics, and Frequency Control, 2017, ISSN: 08853010. DOI: 10.1109/TUFFC. 2016. 2613991.

[30] O. Falou, M. Rui, A. El Kaffas, J. C. Kumaradas, and M. C. Kolios, “The measurement of ultrasound scattering from individual micron-sized objects and its application in single cell scattering", The Journal of the Acoustical Society of America, vol. 128, no. 2, pp. 894-902, 2010, ISSN: 0001-4966. DOI: 10.1121/1.3455795.

[31] T. Segers, E. Gaud, M. Versluis, and P. Frinking, "High-precision acoustic measurements of the nonlinear dilatational elasticity of phospholipid coated monodisperse microbubbles", Soft Matter, 2018, ISSN: 17446848. DOI: 10.1039/c8sm00918j.

[32] J. A. Feshitan, C. C. Chen, J. J. Kwan, and M. A. Borden, "Microbubble size isolation by differential centrifugation", Journal of Colloid and Interface Science, vol. 329, no. 2, pp. 316-324, 2009, ISSN: 00219797. DOI: 10.1016/j.jcis.2008.09.066.

[33] M. J. Borrelli, W. D. O’Brien, L. J. Bernock, H. R. Williams, E. Hamilton, J. Wu, M. L. Oelze, and W. C. Culp, "Production of uniformly sized serum albumin and dextrose microbubbles", Ultrasonics Sonochemistry, 2012, ISSN: 13504177. DOI: 10 . 1016 / j . ultsonch.2011.05.010.

[34] VisualSonics, VisualSonics Vevo 770 High-Resolution Imaging System Manual, 2006.

[35] S. Iradji, "Optimization of subharmonic generation from ultrasound contrast agents at high frequency ultrasound", Master's thesis, Ryerson University, 2008.

[36] A. J. Sojahrood, O. Falou, R. Earl, R. Karshafian, and M. C. Kolios, "Influence of the pressure-dependent resonance frequency on the bifurcation structure and backscattered pressure of ultrasound contrast agents: a numerical investigation", Nonlinear Dynamics, vol. 80, no. 1-2, pp. 889-904, 2015, ISSN: 0924090X. DOI: 10.1007/s11071015-1914-7.

[37] A. J. Sojahrood, "Numerical and experimental investigation of the dynamics of ultrasound contrast agents excited at higher multiples of their natural resonance frequency", Digital Commons @ Ryerson, 2011.

[38] W. Lauterborn, "Numerical investigation of nonlinear oscillations of gas bubbles in liquids", The Journal of the Acoustical Society of America, vol. 59, no. 2, pp. 283-293, 2005, ISSN: 0001-4966. DOI: 10.1121/1.380884.

[39] O. Louisnard and J. González-García, "Acoustic cavitation", in Food Engineering Series, 2011. DOI: $10.1007 / 978-1-4419-7472-3 \_2$.

[40] Y. Gong, M. Cabodi, and T. M. Porter, "Acoustic investigation of pressure-dependent resonance and shell elasticity of lipid-coated monodisperse microbubbles", Applied Physics Letters, 2014, ISSN: 00036951. DOI: 10.1063/1.4865805.

[41] M. A. Parrales, J. M. Fernandez, M. Perez-Saborid, J. A. Kopechek, and T. M. Porter, "Acoustic characterization of monodisperse lipid-coated microbubbles: Relationship between size and shell viscoelastic properties", The Journal of the Acoustical Society of America, 2014, ISSN: 0001-4966. DOI: 10.1121/1.4890643. 
[42] W. Lauterborn and T. Kurz, "Physics of bubble oscillations", Reports on Progress in Physics, vol. 73, no. 10, p. 106501, 2010. DOI: 10.1088/0034-4885/73/10/106501.

[43] R. Varga and F. Hegedus, "Classification of the bifurcation structure of a periodically driven gas bubble", Nonlinear Dynamics, vol. 86, no. 2, pp. 1239-1248, 2016, ISSN: 1573269X. DOI: $10.1007 / \mathrm{s} 11071-016-2960-5$. 